\title{
Deformations of spectral triples and their quantum isometry groups via monoidal equivalences
}

\author{
Liebrecht De Sadeleer ${ }^{1}$
}

Received: 16 March 2016 / Revised: 1 July 2016 / Accepted: 7 September 2016

Published online: 29 November 2016

(C) The Author(s) 2016. This article is published with open access at Springerlink.com

\begin{abstract}
In this paper, we propose a new procedure to deform spectral triples and their quantum isometry groups. The deformation data are a spectral triple $(\mathcal{A}, \mathcal{H}, D)$, a compact quantum group $\mathbb{G}$ acting algebraically and by orientation-preserving isometries on $(\mathcal{A}, \mathcal{H}, D)$ and a unitary fiber functor $\psi$ on $\mathbb{G}$. The deformation procedure is a proper generalization of the cocycle deformation of Goswami and Joardar.
\end{abstract}

Keywords Compact quantum groups $\cdot$ Monoidal equivalence $\cdot$ Noncommutative geometry $\cdot$ Spectral triple $\cdot$ Deformation $\cdot$ Quantum isometry group

Mathematics Subject Classification 58B34 - 46L65 · 81R15

\section{Contents}

1 Introduction . . . . . . . . . . . . . . . . . . . . . . . . . . 674

2 Compact quantum groups and monoidal equivalences . . . . . . . . . . . . . . . . 675

2.1 Compact quantum groups and representations . . . . . . . . . . . . . . . . . . . 675

2.2 Actions of compact quantum groups and the spectral subalgebra . . . . . . . . . . . . . . 677

2.3 Monoidal equivalences between compact quantum groups . . . . . . . . . . . . . . . 678

3 Deformation procedure for spectral triples . . . . . . . . . . . . . . . . . . . . . 6 681

3.1 Spectral triples and compact quantum groups acting on them . . . . . . . . . . . . . 681

3.2 Deformation procedure for spectral triples . . . . . . . . . . . . . . . . . . . . 682

4 Cocycle deformation of spectral triples . . . . . . . . . . . . . . . . . . . . . . 688

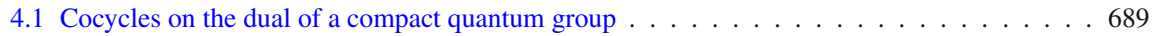

4.2 Algebraic 2-cocycle deformation of a spectral triple . . . . . . . . . . . . . . . . . . . . 690

4.3 Linking dimension-preserving monoidal equivalences with algebraic cocycles . . . . . . . . 692

4.4 Dimension-preserving monoidal deformation is isomorphic to algebraic 2-cocycle deformation 694

Liebrecht De Sadeleer

liebrecht.desadeleer@kuleuven.be

1 Department of Mathematics, KU Leuven, Celestijnenlaan 200B, 3001 Leuven, Belgium 
5 Constructing a non-dimension-preserving example . . . . . . . . . . . . . . . . . . . . 697

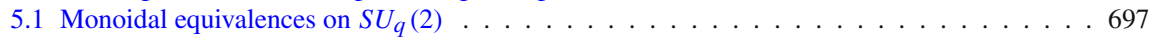

5.2 Monoidal deformation of the Podleś sphere . . . . . . . . . . . . . . . . . . 700

5.2.1 The Podleś sphere, its spectral triple and its quantum isometry group . . . . . . . . . 700

5.2.2 Monoidal deformation of the Podleś sphere . . . . . . . . . . . . . . . . . 702

6 Deformation of the quantum isometry group . . . . . . . . . . . . . . . . . . 703

6.1 Inducing monoidal equivalences on Woronowicz- $C^{*}$-subalgebras . . . . . . . . . . . . . 704

6.2 Inducing monoidal equivalences on supergroups . . . . . . . . . . . . . . . . . . 706

6.3 Deformation of the quantum isometry group . . . . . . . . . . . . . . . 710

6.3.1 Deformation of the universal object in $\mathcal{Q}_{R}(\mathcal{A}, \mathcal{H}, D) \ldots \ldots \ldots$. . . . . . . 710

6.3.2 Deformation of the quantum isometry group . . . . . . . . . . . . . . . 712

6.4 Deformation of the quantum isometry group of the Podleśs sphere . . . . . . . . . . . . . 713

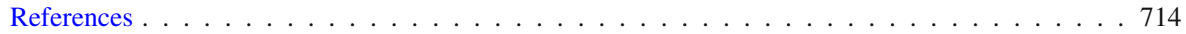

\section{Introduction}

An important source of examples of non-commutative manifolds in the sense of A. Connes (spectral triples, [8]) relies on 2-cocycle deformations. For instance, the socalled 'isospectral deformations' [9] of compact spin manifolds admitting an action of a torus (or an action of the abelian group $\mathbb{R}^{d}$ ) may be seen as a by-product of Rieffel's machinery which, given a $C^{*}$ or Fréchet algebra $\mathbb{A}$ on which $\mathbb{R}^{d}$ acts, produces a one-parameter continuous field of $C^{*}$-algebras $\left\{\mathbb{A}_{\theta}\right\}_{\theta \in \mathbb{R}}$ with $\mathbb{A}_{0}=\mathbb{A}$. The cocycle involved in this case is the usual Moyal 2-cocycle in $\mathbb{R}^{d}$. When $\mathbb{A}$ is the algebra underlying a spectral triple $(\mathbb{A}, \mathcal{H}, D)$, and the action of $\mathbb{R}^{d}$ lifts to an isometric action on $\mathcal{H}$, i.e. an action commuting with $D$, Rieffel machinery produces a new (family of) spectral triple(s) $\left(\mathbb{A}_{\theta}, \mathcal{H}, D\right)$. The paradigm there consists in the noncommutative torus within its metric version.

In the present work, we generalize the deformation procedure through quantum group 2-cocycles (Goswami and Joardar [14]) which is a way to produce new spectral triples from a given one. Our procedure is based on the notion of monoidal equivalence (introduced by Bichon et al. [6]) of (some subgroup of) its quantum isometry group [13]. The generalized procedure here leads to examples that cannot be obtained by 2-cocycle deformations.

The paper is structured as follows. In the first section, we recall some basic material and in the second we describe the deformation procedure. In the third section, we show that 2-cocycle deformations are particular cases of our deformation procedure. Moreover, not all examples are from 2-cocycles: in the fourth section, we give such an example that is not a 2-cocycle deformation, proving our procedure is a proper generalization of the one by Goswami and Joardar. Finally in the last section, we prove that the quantum isometry group of the deformed spectral triple is a certain deformation of the quantum isometry group of the original spectral triple.

Before we end this introduction, we will clarify some notation. Given a Hilbert space $\mathcal{H}$, the inner product $\langle\cdot, \cdot\rangle$ is linear in the second variable. Moreover, for $\xi, \eta \in \mathcal{H}, \xi^{*}$ is the functional $\mathcal{H} \rightarrow \mathbb{C}: \eta \mapsto\langle\xi, \eta\rangle$ and $\xi \eta^{*}$ the rank one operator $\mathcal{H} \rightarrow \mathcal{H}: \zeta \rightarrow$ $\xi\langle\eta, \zeta\rangle$. We will denote by $B(\mathcal{H})$ resp. $\mathcal{K}(\mathcal{H})$ the bounded resp. compact operators on $\mathcal{H}$ and for a bounded or unbounded operator $D$ on $\mathcal{H}, \sigma(D)$ will be used to denote its spectrum. Given a $C^{*}$-algebra $A$, the multiplier algebra of $A$ will be denoted by $\mathcal{M}(A)$, and for a subset $B$ of $A$, we define $\langle B\rangle$ to be the linear span of $B,[B]$ the 
closed linear span, $S(B)$ the *algebra generated by the elements of $B$ and $C^{*}(B)$ the $C^{*}$-subalgebra of $A$ generated by the elements of $B$. Furthermore, we use $\omega_{\xi, \eta}$ to denote the linear functional which maps $a \in B(\mathcal{H})$ to $\langle\xi$, a $\rangle\rangle$ where $\xi, \eta \in \mathcal{H}$, having linearity in the inner product in the second variable.

An algebraic tensor product will be denoted by $\odot$, while the minimal $C^{*}$-algebraic tensor product and a tensor product of Hilbert spaces is denoted by $\otimes$. The tensor product of von Neumann algebras is denoted by $\bar{\otimes}$. We will also use the leg numbering notation in three and multiple tensor products: for $a \in A \otimes A$, we let $a_{12}=a \otimes$ $1_{A}, a_{23}=1_{A} \otimes a_{23}$ and $a_{13}=(\mathrm{id} \otimes \tau)\left(a \otimes 1_{A}\right)$, all three elements in $A \otimes A \otimes A$ where $\tau(a \otimes b)=b \otimes a$.

For a Hopf algebra $H$, the coproduct, counit and antipode will be denoted by $\Delta, \varepsilon$ and $S$ resp. We also use the Sweedler notation $\Delta(h)=h_{(1)} \otimes h_{(2)}$. A left, resp. right $H$-comodule is a vector space $A$ endowed with a linear map $\alpha: A \rightarrow H \odot A$ resp. $\alpha: A \rightarrow A \odot H$ satisfying $(\Delta \otimes \mathrm{id}) \alpha=(\mathrm{id} \otimes \alpha) \alpha$ resp. $(\alpha \otimes \mathrm{id}) \alpha=(\mathrm{id} \otimes \Delta) \alpha$. If $A$ is an algebra and $\alpha$ is multiplicative, it is called a coaction of $H$ on $A$ and $A$ is called an $H$-comodule algebra. If $A$ and $B$ are a right resp. left $H$-comodule algebra with resp. coactions $\alpha$ and $\beta, A \underset{H}{\square} B$ will denote the algebra $\{z \in A \odot B \mid(\alpha \otimes \mathrm{id})(z)=$ $(\mathrm{id} \otimes \beta)(z)\}$.

\section{Compact quantum groups and monoidal equivalences}

We start this section with a short overview of the theory of compact quantum groups. The theory is essentially developed in $[24,26]$ and also explained in [15].

\subsection{Compact quantum groups and representations}

Definition 2.1 ([26]) A compact quantum group $\mathbb{G}$ is a pair $(C(\mathbb{G}), \Delta)$, where $C(\mathbb{G})$ is a unital, separable $C^{*}$-algebra and $\Delta: C(\mathbb{G}) \rightarrow C(\mathbb{G}) \otimes C(\mathbb{G})$ a unital ${ }^{*}$-morphism such that

1. $(\Delta \otimes \mathrm{id}) \Delta=(\mathrm{id} \otimes \Delta) \Delta$,

2. $[\Delta(C(\mathbb{G}))(C(\mathbb{G}) \otimes 1)]=C(\mathbb{G}) \otimes C(\mathbb{G})=[\Delta(C(\mathbb{G}))(1 \otimes C(\mathbb{G}))]$,

implementing coassociativity and the cancellation properties.

Moreover, there exists a unique state $h$ on $C(\mathbb{G})$ which is left and right invariant in the sense that $(\mathrm{id} \otimes h) \Delta(x)=h(x) 1_{C(\mathbb{G})}=(h \otimes \mathrm{id}) \Delta(x)$ for all $x \in C(\mathbb{G})[15,24,26]$. This state is called the Haar state of $\mathbb{G}$. In the classical case that $C(\mathbb{G})=C(G)$ for a classical compact group $G$, the Haar state is the state on $C(G)$ obtained by integrating along the Haar measure.

It is well known that, like compact groups, compact quantum groups have a rich representation theory $[15,24,26]$. A right unitary representation of a compact quantum group $\mathbb{G}=(C(\mathbb{G}), \Delta)$ on a Hilbert space $\mathcal{H}$ is a unitary element $U$ of $\mathcal{M}(\mathcal{K}(\mathcal{H}) \otimes C(\mathbb{G})$ ) satisfying (id $\otimes \Delta) U=U_{12} U_{13}$. Analogously, a left unitary representation of $\mathbb{G}$ on $\mathcal{H}$ is a unitary element $U$ of $\mathcal{M}(C(\mathbb{G}) \otimes \mathcal{K}(\mathcal{H})$ ) satisfying $(\Delta \otimes \mathrm{id}) U=U_{13} U_{23}$. In this paper, all representations will be right representations 
unless indicated otherwise. The dimension of $\mathcal{H}$ is called the dimension of the representation. Identifying $\mathcal{M}(\mathcal{K}(\mathcal{H}) \otimes C(\mathbb{G}))$ with $B\left(\mathcal{H} \otimes C(\mathbb{G})\right.$ ), the $C^{*}$-algebra of $C(\mathbb{G})$-linear adjointable maps on the Hilbert- $C^{*}$-module $\mathcal{H} \otimes C(\mathbb{G})$, we will also see representations as maps $u: \mathcal{H} \rightarrow \mathcal{H} \otimes C(\mathbb{G}): \xi \rightarrow U\left(\xi \otimes 1_{C(\mathbb{G})}\right)$ satisfying that $\langle u(\xi), u(\eta)\rangle_{C(\mathbb{G})}=\langle\xi, \eta\rangle 1_{C(\mathbb{G})},(u \otimes \mathrm{id}) u=(\mathrm{id} \otimes \Delta) u$ and $[u(\xi)(1 \otimes a): \xi \in$ $\mathcal{H}, a \in C(\mathbb{G})]=\mathcal{H} \otimes C(\mathbb{G})$.

Moreover, there is the notion of tensor product of representations: if $U$ and $V$ are representations of a quantum group $\mathbb{G}=(C(\mathbb{G}), \Delta)$ on Hilbert spaces $\mathcal{H}_{1}, \mathcal{H}_{2}$, respectively, the tensor product $U \otimes V$ of $U$ and $V$ is defined as $U \otimes V=U_{13} V_{23} \in$ $\mathcal{M}\left(\mathcal{K}\left(\mathcal{H}_{1} \otimes \mathcal{H}_{2}\right) \otimes C(\mathbb{G})\right)$. Furthermore, we call a representation $U$ of $\mathbb{G}$ on $\mathcal{H}$ irreducible if $\operatorname{Mor}(U, U)=\mathbb{C} 1_{B(\mathcal{H})}$, where

$$
\operatorname{Mor}\left(U^{1}, U^{2}\right):=\left\{S \in B\left(\mathcal{H}_{2}, \mathcal{H}_{1}\right) \mid\left(S \otimes 1_{C(\mathbb{G})}\right) U^{2}=U^{1}\left(S \otimes 1_{C(\mathbb{G})}\right)\right\}
$$

for representations $U_{1}$ and $U_{2}$ on $\mathcal{H}_{1}$ resp. $\mathcal{H}_{2}$. An important result states that every irreducible representation is finite dimensional and that every unitary representation is unitarily equivalent to a direct sum of finite dimensional irreducible representations. Finally, for every irreducible unitary representations, there exist the notion of contragredient representation [15,26].

For a compact quantum group $\mathbb{G}$, we denote by $\operatorname{Irred}(\mathbb{G})$ the set of equivalence classes of irreducible representations of $\mathbb{G}$, and for $x \in \operatorname{Irred}(\mathbb{G})$, we will always take a unitary representative $U^{x} \in B\left(\mathcal{H}_{x}\right) \otimes C(\mathbb{G})$. By $\varepsilon$, we will denote the class of the trivial representation $1_{C(\mathbb{G})}$.

Also for a compact quantum group $\mathbb{G}=(C(\mathbb{G}), \Delta)$ and an equivalence class $x \in \operatorname{Irred}(\mathbb{G})$, we will denote by $\left(\omega_{\xi, \eta} \otimes \operatorname{id}_{C(\mathbb{G})}\right) U^{x}$ a matrix coefficient where $\xi, \eta \in \mathcal{H}$ and define $\mathcal{O}(\mathbb{G})$ to be the linear span of matrix coefficients of all irreducible (hence, finite dimensional) representations of $\mathbb{G}$ :

$$
\mathcal{O}(\mathbb{G})=\left\langle\left(\omega_{\xi, \eta} \otimes \operatorname{id}_{C(\mathbb{G})}\right) U^{x} \mid x \in \operatorname{Irred}(\mathbb{G}), \xi, \eta \in \mathcal{H}_{x}\right\rangle,
$$

even more, the matrix coefficients of the irreducible representations form a basis of $\mathcal{O}(\mathbb{G})$. Note that $\mathcal{O}(\mathbb{G})$ is a unital dense *-subalgebra of $C(\mathbb{G})$ which has, endowed with the restriction of $\Delta$ to $\mathcal{O}(\mathbb{G})$, the structure of a Hopf *-algebra. This is a very nontrivial result obtained in [26]; see also [15]. Also, for a $x \in \operatorname{Irred}(\mathbb{G})$, let $\mathcal{O}(\mathbb{G})_{x}=$ $\left\langle\left(\omega_{\xi, \eta} \otimes \operatorname{id}_{C(\mathbb{G})}\right) U^{x} \mid \xi, \eta \in \mathcal{H}_{x}\right\rangle$. Then, we have $\Delta: \mathcal{O}(\mathbb{G})_{x} \rightarrow \mathcal{O}(\mathbb{G})_{x} \odot \mathcal{O}(\mathbb{G})_{x}$ and $\mathcal{O}(\mathbb{G})_{x}^{*}=\mathcal{O}(\mathbb{G})_{\bar{x}}$.

Definition 2.2 ([3]) Let $\mathbb{G}$ be a compact quantum group. The reduced $C^{*}$-algebra $C_{r}(\mathbb{G})$ is defined as the norm closure of $\mathcal{O}(\mathbb{G})$ in the GNS-representation with respect to the Haar state $h$ of $\mathbb{G}$. The universal $C^{*}$-algebra $C_{u}(\mathbb{G})$ is defined as the $C^{*}$-envelope of $\mathcal{O}(\mathbb{G})$. Note that if $\mathbb{G}$ is the dual of a discrete (classical) group $\Gamma$, we have $C_{r}(\mathbb{G})=$ $C_{r}^{*}(\Gamma), C_{u}(\mathbb{G})=C_{u}^{*}(\Gamma)$.

Remark 2.3 Note that for a given compact quantum group $\mathbb{G}$, we have surjective morphisms between the different completions of $\mathcal{O}(\mathbb{G}): C_{u}(\mathbb{G}) \rightarrow C(\mathbb{G}) \rightarrow C_{r}(\mathbb{G})$. We will think of all these algebras as describing the same quantum group. 
Definition 2.4 Let $\mathbb{G}=\left(C_{u}(\mathbb{G}), \Delta_{\mathbb{G}}\right)$ and $\mathbb{H}=\left(C_{u}(\mathbb{H}), \Delta_{\mathbb{H}}\right)$ be compact quantum groups equipped with their universal $C^{*}$-norms. Suppose moreover that there exists a surjective map $\theta: C_{u}(\mathbb{G}) \rightarrow C_{u}(\mathbb{H})$ satisfying $\Delta_{\mathbb{H}} \circ \theta=(\theta \otimes \theta) \Delta_{\mathbb{G}}$. Then we call $\mathbb{H}$ a quantum subgroup of $\mathbb{G}$. Equivalently, $\mathbb{G}$ is called a quantum supergroup of $\mathbb{H}$.

Definition 2.5 ([15]) Let $\mathbb{G}$ be a compact quantum group. Let

$$
c_{0}(\hat{\mathbb{G}})=\oplus_{x \in \operatorname{Irred}(\mathbb{G})} B\left(\mathcal{H}_{x}\right), \quad \ell^{\infty}(\hat{\mathbb{G}})=\prod_{x \in \operatorname{Irred}(\mathbb{G})} B\left(\mathcal{H}_{x}\right) .
$$

Then we call $\hat{\mathbb{G}}$ the dual quantum group of $\mathbb{G}$ which has the structure of a discrete quantum group (see [20] for the definition and results).

Using the notation $\mathbb{V}=\oplus_{x \in \operatorname{Irred}(\mathbb{G})} U^{x}$, we can define the dual comultiplication

$$
\hat{\Delta}: \ell^{\infty}(\hat{\mathbb{G}}) \rightarrow \ell^{\infty}(\hat{\mathbb{G}}) \bar{\otimes} \ell^{\infty}(\hat{\mathbb{G}}):(\hat{\Delta} \otimes \mathrm{id})(\mathbb{V})=\mathbb{V}_{13} \mathbb{V}_{23}
$$

\subsection{Actions of compact quantum groups and the spectral subalgebra}

Definition 2.6 ([18]) Let $B$ be a unital $C^{*}$-algebra and $\mathbb{G}=(C(\mathbb{G}), \Delta)$ a compact quantum group. A right action of $\mathbb{G}$ on $B$ is a unital $*$-homomorphism $\beta: B \rightarrow$ $B \otimes C(\mathbb{G})$ such that

1. $\left(\beta \otimes \operatorname{id}_{C(\mathbb{G})}\right) \beta=\left(\operatorname{id}_{B} \otimes \Delta\right) \beta$

2. $[\beta(B)(1 \otimes C(\mathbb{G}))]=B \otimes C(\mathbb{G})$.

Analogously, a left action is a unital *-morphism $\beta^{\prime}: B \rightarrow C(\mathbb{G}) \otimes B$ satisfying the analogous conditions. We say that the action is ergodic if $B^{\beta}=\{b \in B \mid \beta(b)=$ $b \otimes 1\}=\mathbb{C} 1_{B}$.

One can choose to call the map in this definition 'a coaction' as it is a coaction of the $C^{*}$-algebra $C(\mathbb{G})$ on $B$. However, we choose to call it an action of the compact quantum group to be compatible with the classical case: if $C(\mathbb{G})=C(G)$ and $B=C(X)$ with $G$ a classical compact group and $X$ a compact space, it is an action of $G$ on $X$.

One can prove that in the case of ergodic actions, there is a unique invariant state on $B$ [7], which we will denote by $\omega$.

Note that the most evident example is a quantum group acting on itself by comultiplication. In that situation, one can check that $\omega=h$.

Using the intimate link between the ergodic action of a compact quantum group on a unital $C^{*}$-algebra and the representations of the quantum group, one has the following result.

Proposition 2.7 ([7]) Let $B$ be a unital $C^{*}$-algebra and $\beta: B \rightarrow C(\mathbb{G}) \otimes B$ a left ergodic action of $\mathbb{G}$ on $B$. Define for every $x \in \operatorname{Irred}(\mathbb{G})$,

$$
K_{x}=\left\{\zeta \in \mathcal{H}_{x} \otimes B \mid U_{12}^{x} \zeta_{13}=\left(\operatorname{id}_{\mathcal{H}_{x}} \otimes \beta\right) \zeta\right\}
$$


and

$$
\mathcal{B}_{x}=\left\langle\left(\xi^{*} \otimes 1_{B}\right) \zeta \mid \zeta \in K_{x}, \xi \in \mathcal{H}_{x}\right\rangle
$$

Then, the spaces $\mathcal{B}_{x}$ with $x \in \operatorname{Irred}(\mathbb{G})$ are called the spectral subspaces of $B$ and

$$
\mathcal{B}=\left\langle\left(\xi^{*} \otimes 1_{B}\right) \zeta \mid x \in \operatorname{Irred}(\mathbb{G}), \zeta \in K_{x}, \xi \in \mathcal{H}_{x}\right\rangle
$$

is a dense unital *-subalgebra of $B$, which we will call the spectral subalgebra of $B$ with respect to $\beta$. Moreover, $\beta_{\left.\right|_{\mathcal{B}}}$ is an algebraic coaction of the Hopf ${ }^{*}$-algebra $(\mathcal{O}(\mathbb{G}), \Delta)$ on $\mathcal{B}$.

Remark 2.8 An action $\beta: B \rightarrow B \otimes C(\mathbb{G})$ of $\mathbb{G}$ on $B$ is called universal if $B$ is the universal $C^{*}$-algebra of $\mathcal{B}$. It is called reduced, if the map (id $\left.\otimes h\right) \beta: B \rightarrow B$ onto the fixed point algebra $B^{\beta}$ is faithful.

In Remark 2.3, we saw that a compact quantum group can be described using different $C^{*}$-algebras, having the same underlying (dense) Hopf*-subalgebra. Similarly here, given an action $\beta: B \rightarrow B \otimes C(\mathbb{G})$ of $\mathbb{G}$ on $B$, passing through $\mathcal{B}$ we can associate to it its universal and reduced $C^{*}$-completions $B_{u}$ and $B_{r}$, and we have surjective morphisms: $B_{u} \rightarrow B \rightarrow B_{r}$.

\subsection{Monoidal equivalences between compact quantum groups}

Definition 2.9 ([6]) Let $\mathbb{G}_{1}=\left(C\left(\mathbb{G}_{1}\right), \Delta_{1}\right)$ and $\mathbb{G}_{2}=\left(C\left(\mathbb{G}_{2}\right), \Delta_{2}\right)$ be two compact quantum groups. $\mathbb{G}_{1}$ and $\mathbb{G}_{2}$ are called monoidally equivalent if there exists a bijection $\varphi: \operatorname{Irred}\left(\mathbb{G}_{1}\right) \rightarrow \operatorname{Irred}\left(\mathbb{G}_{2}\right)$ which satisfies $\varphi\left(\varepsilon_{\mathbb{G}_{1}}\right)=\varepsilon_{\mathbb{G}_{2}}$ together with linear isomorphisms:

$$
\begin{aligned}
\varphi & : \operatorname{Mor}\left(x_{1} \otimes \cdots \otimes x_{r}, y_{1} \otimes \cdots \otimes y_{k}\right) \\
& \rightarrow \operatorname{Mor}\left(\varphi\left(x_{1}\right) \otimes \cdots \otimes \varphi\left(x_{r}\right), \varphi\left(y_{1}\right) \otimes \cdots \otimes \varphi\left(y_{k}\right)\right),
\end{aligned}
$$

satisfying

$$
\begin{aligned}
& \varphi(1)=1, \quad \varphi(S \otimes T)=\varphi(S) \otimes \varphi(T), \\
& \varphi\left(S^{*}\right)=\varphi(S)^{*}, \quad \varphi(S T)=\varphi(S) \varphi(T)
\end{aligned}
$$

whenever the formulas make sense. The collection of maps is called a monoidal equivalence.

Note that this is indeed the usual definition of equivalence between strict monoidal categories, but adapted to the concrete case of the category of representations of a compact quantum group.

Definition 2.10 ([6]) Let $\mathbb{G}=(C(\mathbb{G}), \Delta)$ be a compact quantum group. A unitary fiber functor is a collection of maps $\psi$ such that

- for every $x \in \operatorname{Irred}(\mathbb{G})$, there is a finite dimensional Hilbert space $\mathcal{H}_{\psi(x)}$, 
- there are linear maps

$$
\begin{aligned}
& \psi: \operatorname{Mor}\left(x_{1} \otimes \cdots \otimes x_{k}, y_{1} \otimes \cdots \otimes y_{s}\right) \\
& \quad \rightarrow B\left(\mathcal{H}_{\psi\left(y_{1}\right)} \otimes \cdots \otimes \mathcal{H}_{\psi\left(y_{s}\right)}, \mathcal{H}_{\psi\left(x_{1}\right)} \otimes \cdots \otimes \mathcal{H}_{\psi\left(x_{k}\right)}\right)
\end{aligned}
$$

which satisfy the Eq. (2.1) of Definition 2.9.

Remark 2.11 ([6]) To define a unitary fiber functor it suffices to attach to every $x \in$ $\operatorname{Irred}(\mathbb{G})$ a finite dimensional Hilbert space $\mathcal{H}_{\psi(x)}\left(\mathcal{H}_{\varepsilon}=\mathbb{C}\right)$ and to define the linear maps

$$
\psi: \operatorname{Mor}\left(x_{1} \otimes \cdots \otimes x_{k}, y\right) \rightarrow B\left(\mathcal{H}_{\psi(y)}, \mathcal{H}_{\psi\left(x_{1}\right)} \otimes \cdots \otimes \mathcal{H}_{\psi\left(x_{k}\right)}\right)
$$

for $k=1,2,3$ satisfying

$$
\begin{gathered}
\psi(1)=1 \\
\psi(S)^{*} \psi(T)=\psi\left(S^{*} T\right) \quad \text { if } S \in \operatorname{Mor}(x \otimes y, a), \quad T \in \operatorname{Mor}(x \otimes y, b), \\
(\psi(S) \otimes \text { id }) \psi(T)=\psi((S \otimes \text { id }) T) \quad \text { if } S \in \operatorname{Mor}(x \otimes y, a), \quad T \in \operatorname{Mor}(a \otimes z, b),
\end{gathered}
$$

$($ id $\otimes \psi(S)) \psi(T)=\psi((\operatorname{id} \otimes S) T) \quad$ if $S \in \operatorname{Mor}(x \otimes y, a), \quad T \in \operatorname{Mor}(a \otimes z, b)$,

together with a non-degenerateness condition

$$
\left[\psi(S) \xi \mid a \in \operatorname{Irred}(\mathbb{G}), S \in \operatorname{Mor}(b \otimes c, a), \xi \in \mathcal{H}_{\psi(a)}\right]=\mathcal{H}_{\psi(b)} \otimes \mathcal{H}_{\psi(c)} .
$$

In fact, the notions of unitary fiber functor and monoidal equivalence are equivalent, which is stated in the following proposition, taken from Proposition 3.12 in [6].

Proposition 2.12 Let $\mathbb{G}_{1}$ be a compact quantum group and $\psi$ a unitary fiber functor on it. Then there exists a unique universal compact quantum group $\mathbb{G}_{2}$ with underlying Hopf algebra $\left(\mathcal{O}\left(\mathbb{G}_{2}\right), \Delta_{2}\right)$ with unitary representations $U^{\psi(x)} \in B\left(\mathcal{H}_{\psi(x)}\right) \otimes C\left(\mathbb{G}_{2}\right)$, $x \in \operatorname{Irred}\left(\mathbb{G}_{1}\right)$, such that

1. $U_{13}^{\psi(y)} U_{23}^{\psi(z)}(\psi(S) \otimes 1)=(\psi(S) \otimes 1) U^{\psi(x)}$ for all $S \in \operatorname{Mor}(y \otimes z, x)$,

2. the matrix coefficients of the $U^{\psi(x)}, x \in \operatorname{Irred}\left(\mathbb{G}_{1}\right)$ form a linear basis of $\mathcal{O}\left(\mathbb{G}_{2}\right)$.

Moreover, the set $\left\{U^{\psi(x)} \mid x \in \mathbb{G}_{1}\right\}$ forms a complete set of irreducible representations of $\mathbb{G}_{2}$ and the unitary fiber functor $\psi$ on $\mathbb{G}_{1}$ will induce a monoidal equivalence $\varphi: \mathbb{G}_{1} \rightarrow \mathbb{G}_{2}$.

The following theorems of Bichon et al. will be crucial in our main result. They explain what extra structure a monoidal equivalence induces.

The first theorem follows from Theorem 3.9 and Proposition 3.13 of [6]. 
Theorem 2.13 ([6] $)^{1}$ Let $\mathbb{G}_{1}$ be a compact quantum group and let $\psi$ be a unitary fiber functor on $\mathbb{G}_{1}$. Denote with $\varphi: \mathbb{G}_{1} \rightarrow \mathbb{G}_{2}$ the monoidal equivalence induced by $\psi$ (see previous proposition).

1. There exists a unique unital ${ }^{*}$-algebra $\mathcal{B}$ equipped with a faithful state $\omega$ and unitary elements $X^{x} \in B\left(\mathcal{H}_{\varphi(x)}, \mathcal{H}_{x}\right) \odot \mathcal{B}$ for all $x \in \operatorname{Irred}\left(\mathbb{G}_{1}\right)$ satisfying

(a) $X_{13}^{y} X_{23}^{z}(\varphi(S) \otimes 1)=(S \otimes 1) X^{x}$ for all $S \in \operatorname{Mor}(y \otimes z, x)$,

(b) the matrix coefficients of the $X^{x}$ form a linear basis of $\mathcal{B}$,

(c) $(\mathrm{id} \otimes \omega)\left(X^{x}\right)=0$ if $x \neq \varepsilon$.

2. There exist unique commuting coactions $\beta_{1}: \mathcal{B} \rightarrow \mathcal{O}\left(\mathbb{G}_{1}\right) \odot \mathcal{B}$ and $\beta_{2}: \mathcal{B} \rightarrow$ $\mathcal{B} \odot \mathcal{O}\left(\mathbb{G}_{2}\right)$ satisfying

$$
\left(\mathrm{id} \otimes \beta_{1}\right)\left(X^{x}\right)=U_{12}^{x} X_{13}^{x} \text { and }\left(\mathrm{id} \otimes \beta_{2}\right)\left(X^{x}\right)=X_{12}^{x} U_{13}^{\varphi(x)}
$$

for all $x \in \operatorname{Irred}(\mathbb{G})$. Moreover, $\omega(b) 1_{B}=\left(h \otimes \mathrm{id}_{B}\right) \beta_{1}(b)$.

3. The state $\omega$ is invariant under $\beta_{1}$ and $\beta_{2}$. Denoting by $B_{r}$ the $C^{*}$-algebra generated by $\mathcal{B}$ in the GNS-representation associated with $\omega$ and denoting by $B_{u}$ the universal enveloping $C^{*}$-algebra of $\mathcal{B}$, the Hopf algebraic coactions $\beta_{1}$ and $\beta_{2}$ admit unique extensions to actions of the compact quantum groups on $B_{r}$, resp. $B_{u}$. These actions are reduced, resp. universal and they are ergodic and of full quantum multiplicity (see [6] for the definition).

Definition 2.14 In what follows, we will call $\mathcal{B}$ the $\mathbb{G}_{1}-\mathbb{G}_{2}$-bi-Galois object associated with $\varphi$.

In the spirit of this theorem, we can introduce the notion of isomorphism of unitary fiber functors, which will be equivalent to the isomorphism of the associated bi-Galois objects.

Definition 2.15 (Def. 3.10 in [6]) Let $\psi$ and $\psi^{\prime}$ be two unitary fiber functors on a compact quantum group $\mathbb{G}$. We say they are isomorphic if there exist unitaries $u_{x} \in B\left(\mathcal{H}_{\varphi(x)}, \mathcal{H}_{\psi(x)}\right)$ such that

$$
\psi^{\prime}(S)=\left(u_{y_{1}} \otimes \cdots \otimes u_{y_{k}}\right) \psi(S)\left(u_{x_{1}}^{*} \otimes \cdots \otimes u_{x_{r}}^{*}\right)
$$

for all $S \in \operatorname{Mor}\left(y_{1} \otimes \cdots \otimes y_{k}, x_{1} \otimes \cdots \otimes x_{r}\right)$.

Proposition 2.16 Let $\psi$ and $\psi^{\prime}$ be two unitary fiber functors on a compact quantum group $\mathbb{G}$. Let $\mathcal{B}_{\psi^{\prime}}$ and $\mathcal{B}_{\psi}$ be the associated bi-Galois objects with respective coactions $\beta_{\psi}, \beta_{\psi}^{\prime}$. Then, $\psi$ and $\psi^{\prime}$ are isomorphic as unitary fiber functors if and only if there exists $a^{*}$-isomorphism $\lambda: \mathcal{B}_{\psi} \rightarrow \mathcal{B}_{\psi^{\prime}}$, satisfying $(\lambda \otimes \mathrm{id}) \beta_{\psi}=\beta_{\psi^{\prime}} \lambda$.

Moreover, De Rijdt and Vander Vennet proved in [11] that there exists a bijection between actions of monoidal equivalent compact quantum groups. Indeed, let $\mathbb{G}_{1}$ and

\footnotetext{
1 In the original statement of [6], the coaction $\beta_{1}$ is a right coaction of $\mathcal{O}\left(\mathbb{G}_{1}\right)$, but for what follows, we want a left coaction of $\mathcal{O}\left(\mathbb{G}_{1}\right)$ and a right coaction of $\mathcal{O}\left(\mathbb{G}_{2}\right)$. Applying Bichon's theorem on the inverse monoidal equivalence $\varphi^{\prime}: \mathbb{G}_{2} \rightarrow \mathbb{G}_{1}$, one gets the theorem stated here. Note that, when doing that, we should write $X^{\varphi(x)}, x \in \operatorname{Irred}\left(\mathbb{G}_{1}\right)$, but for notational convenience, we write $X^{x}, x \in \operatorname{Irred}\left(\mathbb{G}_{1}\right)$.
} 
$\mathbb{G}_{2}$ be two compact quantum groups, and $\varphi: \mathbb{G}_{1} \rightarrow \mathbb{G}_{2}$ be a monoidal equivalence between them. Let $\mathcal{B}, \beta_{1}, \beta_{2}, X^{x}$ be as in the previous theorem. Suppose moreover that we have a $C^{*}$-algebra $D_{1}$ and an action $\alpha_{1}: D_{1} \rightarrow D_{1} \otimes C\left(\mathbb{G}_{1}\right)$ of $\mathbb{G}_{1}$ on $D_{1}$. Using the dense Hopf ${ }^{*}$-algebras, we have a coaction $\alpha_{1}: \mathcal{D}_{1} \rightarrow \mathcal{D}_{1} \odot \mathcal{O}\left(\mathbb{G}_{1}\right)$ of $\mathcal{O}\left(\mathbb{G}_{1}\right)$ on $\mathcal{D}_{1}$ and we can define the ${ }^{*}$-algebra:

$$
\mathcal{D}_{2}=\mathcal{D}_{1} \underset{\mathcal{O}\left(\mathbb{G}_{1}\right)}{\square} \mathcal{B}=\left\{a \in \mathcal{D}_{1} \odot \mathcal{B} \mid\left(\alpha_{1} \otimes \operatorname{id}_{\mathcal{B}}\right)(a)=\left(\operatorname{id}_{\mathcal{D}_{1}} \otimes \beta_{1}\right)(a)\right\}
$$

Moreover, in [11], the authors prove that the same construction with the inverse monoidal equivalence $\varphi^{-1}$ will give $\mathcal{D}_{1}$ again up to isomorphism.

Theorem 2.17 Given the data above, there exists an action $\alpha_{2}=\left(\mathrm{id} \otimes \beta_{2}\right)_{\left.\right|_{\mathcal{D}_{2}}}$ on $\mathcal{D}_{2}$. Moreover, if $\alpha_{1}$ is ergodic, $\alpha_{2}$ is ergodic as well.

To end this subsection, we have a look at the inverse monoidal equivalence. We rephrase Proposition 7.6 from [11] in our notations.

Proposition 2.18 Let $\mathbb{G}_{1}$ and $\mathbb{G}_{2}$ be two compact quantum groups and $\varphi: \mathbb{G}_{1} \rightarrow$ $\mathbb{G}_{2}$ a monoidal equivalence with bi-Galois object $\mathcal{B}$. Denote by $\varphi^{-1}: \mathbb{G}_{2} \rightarrow \mathbb{G}_{1}$ the inverse monoidal equivalence with bi-Galois object $\tilde{\mathcal{B}}$ generated by the matrix coefficients of unitaries $Z^{y} \in B\left(\mathcal{H}_{\varphi^{-1}(y)}, \mathcal{H}_{y}\right) \odot \tilde{\mathcal{B}}, y \in \operatorname{Irred}\left(\mathbb{G}_{2}\right)$ and coactions $\delta_{1}: \tilde{B} \rightarrow \tilde{B} \odot \mathcal{O}\left(\mathbb{G}_{1}\right)$ and $\delta_{2}: \tilde{B} \rightarrow \mathcal{O}\left(\mathbb{G}_{2}\right) \odot \tilde{\mathcal{B}}$ such that

$$
\left(\mathrm{id} \otimes \delta_{1}\right) Z^{y}=Z_{12}^{y} U_{13}^{\varphi^{-1}(y)} \quad \text { and } \quad\left(\mathrm{id} \otimes \delta_{2}\right) Z^{y}=U_{12}^{y} Z_{13}^{y} .
$$

Then,

$$
\pi: \mathcal{O}\left(\mathbb{G}_{1}\right) \rightarrow \mathcal{B} \underset{\mathcal{O}\left(\mathbb{G}_{2}\right)}{\square} \tilde{\mathcal{B}} \quad \text { with } \quad(\mathrm{id} \otimes \pi)\left(U^{x}\right)=X_{12}^{x} Z_{13}^{\varphi(x)}
$$

is $a^{*}$-isomorphism intertwining the comultiplication $\Delta_{1}$ with the coaction $\left(\beta_{1} \otimes \mathrm{id}\right)=$ (id $\left.\otimes \delta_{1}\right)$.

\section{Deformation procedure for spectral triples}

Before we start with the description of the deformation procedure, we recapitulate the notion of spectral triples and that of CQG acting on spectral triples.

\subsection{Spectral triples and compact quantum groups acting on them}

Definition 3.1 ([8]) A (compact) spectral triple $(\mathcal{A}, \mathcal{H}, D)$ consists of

1. a unital ${ }^{*}$-algebra $\mathcal{A}$ acting as bounded operators on $\mathcal{H}$,

2. a Hilbert space $\mathcal{H}$,

3. an unbounded self-adjoint operator $D$ on $\mathcal{H}$ with compact resolvent such that $[D, a]$ is bounded for all $a \in \mathcal{A}$. 
Definition 3.2 ([8]) Two spectral triples $\left(\mathcal{A}_{1}, \mathcal{H}_{1}, D_{1}\right)$ and $\left(\mathcal{A}_{2}, \mathcal{H}_{2}, D_{2}\right)$ are called isomorphic, if there exists an isomorphism of Hilbert spaces $\phi: \mathcal{H}_{1} \rightarrow \mathcal{H}_{2}$ and an isomorphism of ${ }^{*}$-algebras $\lambda: \mathcal{A}_{1} \rightarrow \mathcal{A}_{2}$ such that $\phi D_{1}=D_{2} \phi$ and $\phi(a \xi)=$ $\lambda(a) \phi(\xi)$ for arbitrary $\xi \in \mathcal{H}_{1}, a \in \mathcal{A}_{1}$.

In $[4,13]$, Bhowmick and Goswami described how compact quantum groups can act isometrically and be orientation-preserving on a non-commutative manifold, i.e. a spectral triple.

Definition 3.3 ([4]) Let $(\mathcal{A}, \mathcal{H}, D)$ be a compact spectral triple, $\mathbb{G}=(C(\mathbb{G}), \Delta)$ a compact quantum group and $U$ a unitary representation of $\mathbb{G}$ on $\mathcal{H}$. Then, $\mathbb{G}$ is said to act by orientation-preserving isometries on $(\mathcal{A}, \mathcal{H}, D)$ with $U$ if

- for every state $\phi$ on $C(\mathbb{G})$, we have $U_{\phi} D=D U_{\phi}$ where $U_{\phi}:=(\mathrm{id} \otimes \phi)(U)$,

- (id $\otimes \phi) \circ \alpha_{U}(a) \in \mathcal{A}^{\prime \prime}$ for all $a \in \mathcal{A}$ and state $\phi$ on $C(\mathbb{G})$; where $\alpha_{U}(T):=$ $U(T \otimes 1) U^{*}$ for $T \in B(\mathcal{H})$.

This definition is a very strong one: it ensures the existence of a universal object in the category of all compact quantum groups acting by orientation-preserving isometries. However, in some cases, the second condition is too weak: the quantum group representation on $\mathcal{H}$ may behave badly with respect to the algebra $\mathcal{A}$ in the sense that the induced action of the CQG on $\mathcal{A}$ is not a CQG-action on the $C^{*}$-closure of $\mathcal{A}$. This is in some situations a disadvantage. Therefore, we note the following proposition of Goswami, found in [14].

Proposition 3.4 Let $(\mathcal{A}, \mathcal{H}, D)$ and $(C(\mathbb{G}), \Delta, U)$ be as above. Then there exists a unital *-algebra $\mathcal{A}_{1}$ such that

1. $\mathcal{A}_{1}$ is SOT-dense in the von Neumann algebra $M=\mathcal{A}^{\prime \prime}$,

2. $\alpha_{U}$ is algebraic on $\mathcal{A}_{1}$, i.e. $\left(\alpha_{U}\right)_{\left.\right|_{\mathcal{A}_{1}}}: \mathcal{A}_{1} \rightarrow \mathcal{A}_{1} \odot \mathcal{O}(\mathbb{G})$,

3. $[D, a]$ is bounded for every $a \in \mathcal{A}_{1}$,

4. $\left(\mathcal{A}_{1}, \mathcal{H}, D\right)$ is again a spectral triple.

Proof This follows from sections 4.4.3 and 4.4.4 and theorem 4.10 in [14].

Driven by Proposition 3.4, we will use the following definition:

Definition 3.5 Let $(\mathcal{A}, \mathcal{H}, D)$ be a compact spectral triple, $\mathbb{G}=(C(\mathbb{G}), \Delta)$ a compact quantum group and $U$ a unitary representation of $\mathbb{G}$ on $\mathcal{H}$. Then, $\mathbb{G}$ is said to act algebraically and by orientation-preserving isometries on $(\mathcal{A}, \mathcal{H}, D)$ with $U$ if

- for every state $\phi$ on $C(\mathbb{G})$, we have $U_{\phi} D=D U_{\phi}$ where $U_{\phi}:=(\mathrm{id} \otimes \phi)(U)$,

- $\alpha_{U}$ is algebraic on $\mathcal{A}$, i.e. $\left(\alpha_{U}\right)_{\left.\right|_{\mathcal{A}}}: \mathcal{A} \rightarrow \mathcal{A} \odot \mathcal{O}(\mathbb{G})$ where $\alpha_{U}(T):=U(T \otimes 1) U^{*}$ for $T \in B(\mathcal{H})$.

In what follows, we will always work with compact quantum groups acting algebraically on the algebra $\mathcal{A}$.

\subsection{Deformation procedure for spectral triples}

In this subsection, we will describe the actual deformation procedure for spectral triples. The deformation data to start with are: 
- a spectral triple $(\mathcal{A}, \mathcal{H}, D)$ of compact type,

- a compact quantum group $\mathbb{G}_{1}=\left(C\left(\mathbb{G}_{1}\right), \Delta_{1}\right)$ acting algebraically and by orientation-preserving isometries on $(\mathcal{A}, \mathcal{H}, D)$ with a unitary representation $U$ and

- a unitary fiber functor $\psi$ on $\mathbb{G}_{1}$.

The unitary fiber functor will induce a new compact quantum group $\mathbb{G}_{2}$ and a *-algebra $\mathcal{B}$ with left resp. right coaction of $\mathcal{O}\left(\mathbb{G}_{1}\right)$ resp. $\mathcal{O}\left(\mathbb{G}_{2}\right)$. Using this, one can deform the data one by one to obtain a new, deformed, spectral triple on which $\mathbb{G}_{2}$ acts in an appropriate way.

To be more precise, consider the following:

1. As $\psi$ is a unitary fiber functor on $\mathbb{G}_{1}$, following Theorem 2.13 there exists a compact quantum group $\mathbb{G}_{2}$ and a monoidal equivalence $\varphi: \mathbb{G}_{1} \rightarrow \mathbb{G}_{2}$. We will call $\mathbb{G}_{2}$ the deformed quantum group.

2. Let $(\mathcal{B}, \omega)$ be the *-algebra and faithful invariant state associated with $\varphi$ with the coactions

$$
\beta_{1}: \mathcal{B} \rightarrow \mathcal{O}\left(\mathbb{G}_{1}\right) \odot \mathcal{B} \text { and } \beta_{2}: \mathcal{B} \rightarrow \mathcal{B} \odot \mathcal{O}\left(\mathbb{G}_{2}\right)
$$

3. Let $X^{x} \in B\left(\mathcal{H}_{\varphi(x)}, \mathcal{H}_{x}\right) \odot \mathcal{B}$ be the unitaries such that

$$
\left(\mathrm{id} \otimes \beta_{1}\right) X^{x}=U_{12}^{x} X_{13}^{x} \quad \text { and } \quad\left(\mathrm{id} \otimes \beta_{2}\right) X^{x}=X_{12}^{x} U_{13}^{\varphi(x)}
$$

4. Let $u: \mathcal{H} \rightarrow \mathcal{H} \otimes C\left(\mathbb{G}_{1}\right): \xi \mapsto U(\xi \otimes 1)$ be the representation of $\mathbb{G}_{1}$ on $\mathcal{H}$ and denote by $\alpha=\operatorname{ad}_{U}: \mathcal{A} \rightarrow \mathcal{A} \odot \mathcal{O}\left(\mathbb{G}_{1}\right): a \rightarrow U\left(a \otimes 1_{C\left(\mathbb{G}_{1}\right)}\right) U^{*}$ the algebraic coaction of $\mathcal{O}\left(\mathbb{G}_{1}\right)$ on $\mathcal{A}$.

We start by introducing the deformed data and proving some basic facts about them.

Proposition 3.6 Defining $L^{2}(\mathcal{B})$ to be the GNS space of $\mathcal{B}$ with respect to $\omega$ and $\Lambda: \mathcal{B} \rightarrow L^{2}(\mathcal{B})$ the GNS map, we have that :

1. there exists a unitary representation $\beta_{1}^{\prime}$ of $C\left(\mathbb{G}_{1}\right)$ on $L^{2}(\mathcal{B})$ such that $\beta_{1}^{\prime}(\Lambda(b))=$ $(\mathrm{id} \otimes \Lambda)\left(\beta_{1}(b)\right)$;

2. $\beta_{1}^{\prime}$ is ergodic, i.e. if $\xi \in L^{2}(\mathcal{B})$ such that $\beta_{1}^{\prime}(\xi)=1 \otimes \xi$, then $\xi \in \mathbb{C} \Lambda\left(1_{\mathcal{B}}\right)$.

3. The vector space $\mathcal{H} \underset{C\left(\mathbb{G}_{1}\right)}{\otimes} L^{2}(\mathcal{B})=\left\{\xi \in \mathcal{H} \otimes L^{2}(\mathcal{B}) \mid U_{12} \xi_{13}=\left(\operatorname{id}_{\mathcal{H}} \otimes \beta_{1}^{\prime}\right)(\xi)\right\}$ is a Hilbert space which we denote by $\tilde{\mathcal{H}}$.

Proof 1. As $\omega$ is faithful on $\mathcal{B}, \Lambda$ is injective and hence $\beta_{1}^{\prime}$ is well defined on $\Lambda(\mathcal{B})$. Using that $\beta_{1}$ is a well-defined coaction of $\mathcal{O}\left(\mathbb{G}_{1}\right)$ and that $\omega$ is $\beta_{1}$-invariant, $\beta_{1}^{\prime}$ can be extended to a unitary representation on $L^{2}(\mathcal{B})$.

2. Let $\xi$ be an element in $L^{2}(\mathcal{B})$ satisfying $\beta_{1}^{\prime}(\xi)=1 \otimes \xi$. Take a sequence $\left(b_{n}\right)_{n}$ in $\mathcal{B}$ with $\Lambda\left(b_{n}\right) \rightarrow \xi$ in $L^{2}$-norm, then for $P=\left(h \otimes \operatorname{id}_{L^{2}(\mathcal{B})}\right) \beta_{1}^{\prime}$ we see that $P\left(\Lambda\left(b_{n}\right)\right) \rightarrow P(\xi)=\xi$ since $P$ is a continuous operator on $L^{2}(\mathcal{B})$. Seeing that $P\left(\Lambda\left(b_{n}\right)\right)=\omega\left(b_{n}\right) \Lambda\left(1_{\mathcal{B}}\right) \in \mathbb{C} 1_{\mathcal{B}}$ concludes this proof. 
3. It follows directly that $\mathcal{H} \underset{C\left(\mathbb{G}_{1}\right)}{\otimes} L^{2}(\mathcal{B})$ is a vector subspace of the tensor product Hilbert space $\mathcal{H} \otimes L^{2}(\mathcal{B})$. As the representations $u$ and $\beta_{1}^{\prime}$ of $\mathbb{G}_{1}$ on $\mathcal{H}$ resp. $L^{2}(\mathcal{B})$ are continuous and $\mathcal{H} \underset{C\left(\mathbb{G}_{1}\right)}{\otimes} L^{2}(\mathcal{B})$ is the kernel of $u \otimes \operatorname{id}_{L^{2}(\mathcal{B})}-\operatorname{id}_{\mathcal{H}} \otimes \beta_{1}^{\prime}$, $\mathcal{H} \underset{C\left(\mathbb{G}_{1}\right)}{\otimes} L^{2}(\mathcal{B})$ is complete.

Proposition 3.7 We have

1. $\mathcal{H}_{x} \underset{C\left(\mathbb{G}_{1}\right)}{\otimes} L^{2}(\mathcal{B})$ is isomorphic with $\mathcal{H}_{\varphi(x)}$ for all $x \in \operatorname{Irred}\left(\mathbb{G}_{1}\right)$. 2.

$$
\mathcal{H} \underset{C\left(\mathbb{G}_{1}\right)}{\bigotimes} L^{2}(\mathcal{B})=\bigoplus_{\lambda \in \sigma(D)} V_{\lambda} \underset{C\left(\mathbb{G}_{1}\right)}{\bigotimes} L^{2}(\mathcal{B})
$$

where $V_{\lambda}$ is the eigenspace of $\lambda \in \sigma(D)$.

3. $V_{\lambda} \underset{C\left(\mathbb{G}_{1}\right)}{\otimes} L^{2}(\mathcal{B})$ is finite dimensional for each $\lambda \in \sigma(D)$.

Motivated by the first fact, we will call $\mathcal{H}_{\varphi(x)}$ the deformation of $\mathcal{H}_{x}$ for $x \in \operatorname{Irred}\left(\mathbb{G}_{1}\right)$.

Proof 1. Analogously to $\mathcal{H} \underset{C\left(\mathbb{G}_{1}\right)}{\otimes} L^{2}(\mathcal{B})$, we define for $x \in \operatorname{Irred}\left(\mathbb{G}_{1}\right)$

$$
\mathcal{H}_{x} \underset{C\left(\mathbb{G}_{1}\right)}{\otimes} L^{2}(\mathcal{B})=\left\{z \in \mathcal{H}_{x} \otimes L^{2}(\mathcal{B}) \mid U_{12}^{x} z_{13}=\left(\mathrm{id} \otimes \beta_{1}^{\prime}\right)(z)\right\}
$$

Now note that, for $x \in \operatorname{Irred}\left(\mathbb{G}_{1}\right)$ and $\xi \in \mathcal{H}_{\varphi(x)}, X^{x}\left(\xi \otimes \Lambda_{(1 \mathcal{B}}\right) \in$ $\mathcal{H}_{x} \underset{C\left(\mathbb{G}_{1}\right)}{\otimes} L^{2}(\mathcal{B})$, and for $z \in \mathcal{H}_{x} \underset{C\left(\mathbb{G}_{1}\right)}{\bigotimes} L^{2}(\mathcal{B}),\left(\operatorname{id}_{\mathcal{H}_{\varphi(x)}} \otimes \omega_{1}^{\prime}\right)\left(X^{x *} z\right) \in \mathcal{H}_{\varphi(x)}$ where $\omega_{1}^{\prime}: L^{2}(\mathcal{B}) \rightarrow \mathbb{C}: \eta \mapsto\langle\Lambda(1), \eta\rangle$. Hence, we can define the following maps:

$$
\begin{gathered}
f_{x}: \mathcal{H}_{\varphi(x)} \rightarrow \mathcal{H}_{x} \underset{C\left(\mathbb{G}_{1}\right)}{\otimes} L^{2}(\mathcal{B}): \xi \mapsto X^{x}\left(\xi \otimes \Lambda\left(1_{\mathcal{B}}\right)\right), \\
\left.g_{x}: \mathcal{H}_{x} \underset{C\left(\mathbb{G}_{1}\right)}{\otimes} L^{2}(\mathcal{B}) \rightarrow \mathcal{H}_{\varphi(x)}: z \mapsto \operatorname{id}_{\mathcal{H}_{\varphi(x)}} \otimes \omega_{1}^{\prime}\right)\left(X^{x *} z\right)
\end{gathered}
$$

Using that $\beta_{1}^{\prime}$ is ergodic (Proposition 3.6(2)), one can check that $g_{x}(z) \otimes \Lambda\left(1_{\mathcal{B}}\right)=$ $X^{x *} z$ which ensures that $f_{x}$ and $g_{x}$ are inverse to each other. Finally, using that $X^{x}$ is unitary, it is easy to see that $f_{x}$ and $g_{x}$ are also unitary.

2. Note first that as $D$ has compact resolvent, there exists a sequence of real eigenvalues $\left(\lambda_{n}\right)_{n}$ with finite dimensional eigenspaces and such that $\lim _{n \rightarrow \infty} \lambda_{n}=\infty$. Hence, we have $\mathcal{H}=\bigoplus_{\lambda \in \sigma(D)} V_{\lambda}$ and also $\mathcal{H} \otimes L^{2}(\mathcal{B})=\bigoplus_{\lambda \in \sigma(D)} V_{\lambda} \otimes L^{2}(\mathcal{B})$. As $U$ and $D$ commute, there is a subrepresentation $U_{\lambda}$ of $U$ on $V_{\lambda}$ for every eigenvalue $\lambda$, such that for $V_{\lambda} \underset{C}{\otimes} L^{2}(\mathcal{B}):=\left\{\xi \in V_{\lambda} \otimes L^{2}(\mathcal{B}) \mid\left(U_{\lambda}\right)_{12} \xi_{13}=\left(\mathrm{id} \otimes \beta_{1}^{\prime}\right) \xi\right\}$, we have

$$
\mathcal{H} \underset{C\left(\mathbb{G}_{1}\right)}{\otimes} L^{2}(\mathcal{B})=\bigoplus_{\lambda \in \sigma(D)} V_{\lambda} \underset{C\left(\mathbb{G}_{1}\right)}{\otimes} L^{2}(\mathcal{B})
$$


3. Finally, decomposing $U_{\lambda}$ into irreducible representations of $\mathbb{G}_{1}$, we have $V_{\lambda}=$ $\mathcal{H}_{x_{1}} \oplus \cdots \oplus \mathcal{H}_{x_{l}}$ for some $l \in \mathbb{N}, x_{i} \in \operatorname{Irred}\left(\mathbb{G}_{1}\right)$. Hence,

$$
\begin{aligned}
V_{\lambda} \underset{C\left(\mathbb{G}_{1}\right)}{\otimes} L^{2}(\mathcal{B}) & =\left(\mathcal{H}_{x_{1}} \oplus \cdots \oplus \mathcal{H}_{x_{l}}\right) \underset{C\left(\mathbb{G}_{1}\right)}{\otimes} L^{2}(\mathcal{B}) \\
& =\left(\mathcal{H}_{x_{1}} \underset{C\left(\mathbb{G}_{1}\right)}{\otimes} L^{2}(\mathcal{B})\right) \oplus \cdots \oplus\left(\mathcal{H}_{x_{l}} \underset{C\left(\mathbb{G}_{1}\right)}{\otimes} L^{2}(\mathcal{B})\right) \\
& =\mathcal{H}_{\varphi\left(x_{1}\right)} \oplus \cdots \oplus \mathcal{H}_{\varphi\left(x_{l}\right)},
\end{aligned}
$$

where we used the first statement of this proposition. This last direct sum of finite dimensional Hilbert spaces implies $V_{\lambda} \underset{C\left(\mathbb{G}_{1}\right)}{\otimes} L^{2}(\mathcal{B})$ to be finite dimensional.

Proposition 3.8 $D \otimes \mathrm{id}_{L^{2}(\mathcal{B})}$ restricts to an unbounded self-adjoint operator $\tilde{D}$ on $\tilde{\mathcal{H}}=\mathcal{H} \underset{\mathbb{G}_{1}}{\otimes} L^{2}(\mathcal{B})$ of compact resolvent.

Proof As $D$ has compact resolvent, its restriction $D_{\lambda}$ to $V_{\lambda}$ is multiplication with $\lambda$ for every $\lambda$ in the spectrum. Therefore, $D_{\lambda} \otimes$ id can be restricted to $V_{\lambda} \underset{C\left(\mathbb{G}_{1}\right)}{\otimes} L^{2}(\mathcal{B}) \subset V_{\lambda} \otimes$ $L^{2}(\mathcal{B})$. Taking the direct sum, we get an unbounded operator $\tilde{D}$ on $\mathcal{H} \underset{C\left(\mathbb{G}_{1}\right)}{\otimes} L^{2}(\mathcal{B})$ with domain $\left\{\left.\left(\xi_{n}\right)_{n} \in \bigoplus_{\lambda_{n} \in \sigma(D)} V_{\lambda_{n}} \underset{C\left(\mathbb{G}_{1}\right)}{\otimes} L^{2}(\mathcal{B})\left|\sum_{n}\right| \lambda_{n}\right|^{2}\left\|\xi_{n}\right\|^{2}<\infty\right\}$. By construction, we have $\tilde{D}=\sum_{\lambda \in \sigma(D)} \lambda\left(P_{\lambda} \otimes\right.$ id $)$, where $P_{\lambda}$ is the projection $\mathcal{H} \rightarrow V_{\lambda}$. Hence, it has compact resolvent by proposition 3.7(3) and self-adjoint as $D$ is self-adjoint. Moreover, as $\mathcal{H} \otimes L^{2}(\mathcal{B})=\bigoplus_{\lambda \in \sigma(D)} V_{\lambda} \otimes L^{2}(\mathcal{B})$, it is the restriction of

$$
D \otimes \mathrm{id}_{L^{2}(\mathcal{B})}=\bigoplus_{\lambda \in \sigma(D)} D_{\lambda} \otimes \mathrm{id}_{L^{2}(\mathcal{B})}: \bigoplus_{\lambda \in \sigma(D)} V_{\lambda} \otimes L^{2}(\mathcal{B}) \rightarrow \bigoplus_{\lambda \in \sigma(D)} V_{\lambda} \otimes L^{2}(\mathcal{B})
$$

to $\mathcal{H} \underset{C\left(\mathbb{G}_{1}\right)}{\otimes} L^{2}(\mathcal{B})$, concluding the proof.

Proposition 3.9 Define $\tilde{\mathcal{A}}=\mathcal{A} \underset{\mathcal{O}\left(\mathbb{G}_{1}\right)}{\square} \mathcal{B}:=\left\{z \in \mathcal{A} \odot \mathcal{B} \mid\left(\alpha \otimes \operatorname{id}_{\mathcal{B}}\right)(z)=\right.$ $\left.\left(\operatorname{id}_{\mathcal{A}} \otimes \beta_{1}\right)(z)\right\}$. Then $\tilde{\mathcal{A}}$ is a ${ }^{*}$-algebra endowed with a coaction $\alpha_{2}=\left(\operatorname{id} \otimes \beta_{2}\right)_{\tilde{\mathcal{A}}_{\tilde{A}}}$ : $\tilde{\mathcal{A}} \rightarrow \tilde{\mathcal{A}} \odot \mathcal{O}\left(\mathbb{G}_{2}\right)$ of $\mathcal{O}\left(\mathbb{G}_{2}\right)$. Moreover, $\tilde{\mathcal{A}}$ acts by bounded operators on $\tilde{\mathcal{H}}:$ for $z \in \tilde{\mathcal{A}}$, we have $\tilde{L_{z}}: \tilde{\mathcal{H}} \rightarrow \tilde{\mathcal{H}}: v \mapsto z v$ by multiplication on $\mathcal{B}$ and action of $\mathcal{A}$ on $\mathcal{H}$ as a bounded operator on $\tilde{\mathcal{H}}$.

Proof The first statement is an application of Theorem 2.17. For the second, note that $\tilde{\mathcal{A}} \subset \mathcal{A} \odot \mathcal{B}$ and $\mathcal{A} \odot \mathcal{B}$ act by bounded operators on $\mathcal{H} \otimes L^{2}(\mathcal{B})$. Hence, it suffices to prove that $\tilde{\mathcal{A}}$ leaves $\tilde{\mathcal{H}}$ invariant. Indeed, we have for $a \in \tilde{\mathcal{A}}, \xi \in \tilde{\mathcal{H}}$,

$$
\begin{aligned}
\left(\operatorname{id}_{\mathcal{H}} \otimes \beta_{1}^{\prime}\right)(a \xi) & =\left(\operatorname{id}_{\mathcal{A}} \otimes \beta_{1}\right)(a)\left(\operatorname{id}_{\mathcal{H}} \otimes \beta_{1}^{\prime}\right)(\xi) \\
& =\left(\alpha \otimes \operatorname{id}_{\mathcal{B}}\right)(a) U_{12} \xi_{13}=U_{12} a_{13} U_{12}^{*} U_{12} \xi_{13}=U_{12}(a \xi)_{13} .
\end{aligned}
$$


Theorem $3.10(\tilde{\mathcal{A}}, \tilde{\mathcal{H}}, \tilde{D})$ constitutes a spectral triple.

Proof Combining all the previous propositions, it suffices to prove that the commutator of $\tilde{D}$ with an element $a \in \tilde{\mathcal{A}}$ is bounded. For that, we will first prove that $\tilde{\mathcal{A}}$ leaves the domain of $\tilde{D}$ invariant and secondly we will prove that the commutator of $\tilde{D}$ and an arbitrary $a \in \tilde{\mathcal{A}}$ is bounded. Let $z$ be an arbitrary element in $\mathcal{A} \odot \mathcal{B}$ and let $\xi$ be an arbitrary nonzero vector in $\operatorname{dom}(D \otimes \mathrm{id})$. We will prove $z \xi \in \operatorname{dom}(D \otimes \mathrm{id})$. As $\xi \in \operatorname{dom}(D \otimes \mathrm{id})$, there exists a sequence $\xi_{n}$ in $\operatorname{dom}(D) \odot L^{2}(\mathcal{B})$ such that simultaneously $\xi_{n} \rightarrow \xi$ and $(D \otimes \mathrm{id}) \xi_{n} \rightarrow(D \otimes \mathrm{id}) \xi$ for $n \rightarrow \infty$. Note that as $\mathcal{A}$ leaves the domain of $D$ invariant, $\mathcal{A} \odot \mathcal{B}$ leaves the core $\operatorname{dom}(D) \odot L^{2}(\mathcal{B})$ of $D \otimes$ id invariant and hence $z \xi_{n} \in \operatorname{dom}(D) \odot L^{2}(\mathcal{B})$ for all $n$. Moreover, as $\mathcal{A}$ has bounded commutator with $D$, one can prove that $[D \otimes \mathrm{id}, z]$ is bounded on $\operatorname{dom}(D) \odot L^{2}(\mathcal{B})$ and $(D \otimes \mathrm{id}) z\left(\xi_{n}\right)_{n}$ is a Cauchy sequence and thus converging. As $z \xi_{n}$ is an element of the core converging to $z \xi$ and $\left((D \otimes \mathrm{id}) z\left(\xi_{n}\right)\right)_{n}$ converges, we know that $z \xi \in \operatorname{dom}(D \otimes \mathrm{id})$ and $(D \otimes \mathrm{id}) z \xi_{n} \rightarrow(D \otimes \mathrm{id}) z \xi$. We can conclude that $(\mathcal{A} \odot \mathcal{B})(\operatorname{dom}(D \otimes \mathrm{id})) \subset$ $\operatorname{dom}(D \otimes \mathrm{id})$ and it follows directly that $\tilde{\mathcal{A}}(\operatorname{dom}(\tilde{D})) \subset \operatorname{dom}(\tilde{D})$.

Finally, we prove that $\tilde{D} z-z \tilde{D}$ is indeed bounded on the domain of $\tilde{D}$. Let $\xi \in$ $\operatorname{dom}(\tilde{D})$ be arbitrary and take a sequence $\xi_{n} \rightarrow \xi$ in $\operatorname{dom}(D) \odot L^{2}(\mathcal{B})$. Then we know from above that simultaneously

$$
\begin{aligned}
& (D \otimes \mathrm{id}) z \xi_{n} \rightarrow(D \otimes \mathrm{id}) z \xi, \\
& z(D \otimes \mathrm{id}) \xi_{n} \rightarrow z(D \otimes \mathrm{id}) \xi
\end{aligned}
$$

and that $[D \otimes \mathrm{id}, z]$ is bounded on $\operatorname{dom}(D) \odot L^{2}(\mathcal{B})$. Combining that, one can prove that indeed $\tilde{D} z-z \tilde{D}$ is bounded on the domain.

Theorem 3.11 There exists a unitary representation $\tilde{U}$ of $C\left(\mathbb{G}_{2}\right)$ on $\mathcal{H} \underset{C\left(\mathbb{G}_{1}\right)}{\otimes} L^{2}(\mathcal{B})$ such that $\mathbb{G}_{2}$ acts algebraically and by orientation-preserving isometries on $(\tilde{\mathcal{A}}, \tilde{\mathcal{H}}, \tilde{D})$ with $\tilde{U}$.

Proof Using the coaction $\beta_{2}: \mathcal{B} \rightarrow \mathcal{B} \odot \mathcal{O}\left(\mathbb{G}_{2}\right)$ and the CQG-action $\beta_{2}: B_{u} \rightarrow$ $B_{u} \otimes C\left(\mathbb{G}_{2}\right)$, one can construct, along the lines of Lemma 5 in [7] and the discussion above it, a representation $\tilde{U}_{0} \in \mathcal{M}\left(\mathcal{K}\left(L^{2}(\mathcal{B})\right) \otimes C\left(\mathbb{G}_{2}\right)\right)$ such that

$$
\tilde{U}_{0}(\Lambda(b) \otimes a)=\left(\Lambda \otimes \operatorname{id}_{C\left(\mathbb{G}_{2}\right)}\right)\left(\beta_{2}(b)\left(1_{\mathcal{B}} \otimes a\right)\right)
$$

Moreover, we know this is a unitary representation and furthermore,

$$
\beta_{2}(b)=\tilde{U}_{0}(b \otimes \mathrm{id}) \tilde{U}_{0}^{*} .
$$

Now, one can prove that $\mathrm{id}_{\mathcal{H}} \otimes \tilde{U}_{0} \in \mathcal{M}\left(\mathcal{K}\left(\mathcal{H} \otimes L^{2}(\mathcal{B})\right) \otimes C\left(\mathbb{G}_{2}\right)\right)$ restricts to a representation $\tilde{U} \in \mathcal{M}\left(\mathcal{K}\left(\mathcal{H} \underset{C\left(\mathbb{G}_{1}\right)}{\otimes} L^{2}(\mathcal{B})\right) \otimes C\left(\mathbb{G}_{2}\right)\right)$. Indeed, as $\beta_{1}$ and $\beta_{2}$ commute, one has $\left(\beta_{1}^{\prime} \otimes \operatorname{id}_{C\left(\mathbb{G}_{2}\right)}\right) \tilde{U}_{0}=\left(\operatorname{id}_{C\left(\mathbb{G}_{1}\right)} \otimes \tilde{U}_{0}\right)\left(\beta_{1}^{\prime} \otimes \operatorname{id}_{C\left(\mathbb{G}_{2}\right)}\right)$, and hence for 
$\xi \in \mathcal{H} \underset{C\left(\mathbb{G}_{1}\right)}{\bigotimes} L^{2}(\mathcal{B})$ and $a \in C\left(\mathbb{G}_{2}\right)$, one has

$$
\begin{aligned}
& \left(\mathrm{id}_{\mathcal{H}} \otimes \beta_{1}^{\prime} \otimes \mathrm{id}_{C\left(\mathbb{G}_{2}\right)}\right)\left(\mathrm{id}_{\mathcal{H}} \otimes \tilde{U}_{0}\right)(\xi \otimes a) \\
& \quad=\left(\mathrm{id}_{\mathcal{H}} \otimes \mathrm{id}_{C\left(\mathbb{G}_{1}\right)} \otimes \tilde{U}_{0}\right)\left(\mathrm{id}_{\mathcal{H}} \otimes \beta_{1}^{\prime} \otimes \mathrm{id}_{C\left(\mathbb{G}_{2}\right)}\right)(\xi \otimes a) \\
& =\left(\mathrm{id}_{\mathcal{H}} \otimes \mathrm{id}_{C\left(\mathbb{G}_{1}\right)} \otimes \tilde{U}_{0}\right)\left(U \otimes \mathrm{id}_{L^{2}(\mathcal{B})} \otimes \mathrm{id}_{C\left(\mathbb{G}_{2}\right)}\right)\left(\xi_{13} \otimes a\right) \\
& =\left(U \otimes \mathrm{id}_{L^{2}(\mathcal{B})} \otimes \operatorname{id}_{C\left(\mathbb{G}_{2}\right)}\right)\left(\left(\operatorname{id}_{\mathcal{H}} \otimes \tilde{U}_{0}\right)(\xi \otimes a)\right)_{134} .
\end{aligned}
$$

Then it suffices to prove that $\tilde{U}$ commutes with the Dirac operator of the deformed spectral triple and that there is a coaction of $\mathcal{O}\left(\mathbb{G}_{2}\right)$ on $\tilde{\mathcal{A}}$. As $\tilde{D}$ is the restriction of $D \otimes \mathrm{id}_{L^{2}(B)}$ and $\tilde{U}$ is the restriction of $\operatorname{id}_{\mathcal{H}} \otimes \tilde{U}_{0}$, it follows directly that they commute. Using Theorem 2.17, we know that, given the coaction

$$
\alpha_{1}=\operatorname{ad}_{U}: \mathcal{A} \rightarrow \mathcal{A} \odot \mathcal{O}\left(\mathbb{G}_{1}\right): a \rightarrow U\left(a \otimes \operatorname{id}_{\mathcal{A}}\right) U^{*},
$$

there is a coaction $\alpha_{2}: \tilde{\mathcal{A}} \rightarrow \tilde{\mathcal{A}} \odot \mathcal{O}\left(\mathbb{G}_{2}\right): z \rightarrow\left(\operatorname{id}_{\mathcal{A}} \otimes \beta_{2}\right)(z)$. Using (3.2), $\alpha_{2}=$ $\operatorname{id}_{\mathcal{A}} \otimes \operatorname{ad}_{\tilde{U}_{0}}$ and regarding elements of $\mathcal{A}$ as operators on $\mathcal{H}$, we have $\alpha_{2}=\operatorname{ad}_{\tilde{U}}$.

Theorem (Main result) 3.12 Let $(\mathcal{A}, \mathcal{H}, D)$ be a compact spectral triple and let $\mathbb{G}_{1}=\left(C\left(\mathbb{G}_{1}\right), \Delta_{1}\right)$ be a compact quantum group acting algebraically and by orientation-preserving isometries on $(\mathcal{A}, \mathcal{H}, D)$ with a unitary representation $U$. Moreover, let $\psi$ be a unitary fiber functor on $\mathbb{G}_{1}$.

Then there exist a spectral triple $(\tilde{\mathcal{A}}, \tilde{\mathcal{H}}, \tilde{D})$, a compact quantum group $\mathbb{G}_{2}=$ $\left(C\left(\mathbb{G}_{2}\right), \Delta_{2}\right)$ monoidally equivalent to $\mathbb{G}_{1}$ and a unitary representation $\tilde{U}$ of $\mathbb{G}_{2}$ on $\tilde{\mathcal{H}}$ such that the monoidal equivalence is associated with $\psi$ and $\mathbb{G}_{2}$ acts algebraically and by orientation-preserving isometries on the new spectral triple with $\tilde{U}$.

Denoting $\mathcal{B}$ to be the $\left(\mathbb{G}_{1}-\mathbb{G}_{2}\right)$-bi-Galois object, one has

$$
\tilde{\mathcal{A}}=\mathcal{A} \underset{\mathcal{O}\left(\mathbb{G}_{1}\right)}{\square} \mathcal{B}, \quad \tilde{\mathcal{H}}=\mathcal{H} \underset{C\left(\mathbb{G}_{1}\right)}{\otimes} L^{2}(\mathcal{B}), \quad \tilde{D}=\left.\left(D \otimes \mathrm{id}_{L^{2}(\mathcal{B})}\right)\right|_{\tilde{\mathcal{H}}}
$$

In what follows, we will call this deformation procedure 'monoidal deformation'.

To end this section, we will show that via the inverse monoidal equivalence on the deformed quantum group and spectral triple, one can obtain the original data again.

Theorem 3.13 Let $(\mathcal{A}, \mathcal{H}, D)$ be a spectral triple, $\mathbb{G}_{1}$ a compact quantum group acting algebraically and by orientation-preserving isometries on $(\mathcal{A}, \mathcal{H}, D)$. Let $\psi$ be a unitary fiber functor, inducing a monoidal equivalence $\varphi: \mathbb{G}_{1} \rightarrow \mathbb{G}_{2}$ with bi-Galois object $\mathcal{B}$. Denote by $\varphi^{-1}: \mathbb{G}_{2} \rightarrow \mathbb{G}_{1}$ the inverse monoidal equivalence with bi-Galois object $\tilde{\mathcal{B}}$. Then,

$$
\left(\mathcal{A} \underset{\mathcal{O}\left(\mathbb{G}_{1}\right)}{\square} \mathcal{B} \underset{\mathcal{O}\left(\mathbb{G}_{2}\right)}{\square} \tilde{\mathcal{B}}, \mathcal{H} \underset{C\left(\mathbb{G}_{1}\right)}{\otimes} L^{2}(\mathcal{B}) \underset{C\left(\mathbb{G}_{2}\right)}{\otimes} L^{2}(\tilde{\mathcal{B}}), D \otimes \operatorname{id}_{L^{2}(\mathcal{B})} \otimes \operatorname{id}_{L^{2}(\tilde{\mathcal{B}})}\right)
$$

is isomorphic with $(\mathcal{A}, \mathcal{H}, D)$ as spectral triples (Definition 3.2). 
Proof From Proposition 2.18, one obtains the following *-isomorphisms:

$$
\mathcal{A} \stackrel{\alpha_{U}}{\rightarrow} \mathcal{A} \underset{\mathcal{O}\left(\mathbb{G}_{1}\right)}{\square} \mathcal{O}\left(\mathbb{G}_{1}\right) \stackrel{\operatorname{id} \otimes \pi}{\rightarrow} \mathcal{A} \underset{\mathcal{O}\left(\mathbb{G}_{1}\right)}{\square} \mathcal{\mathcal { O } ( \mathbb { G } _ { 2 } )} \underset{\mathcal{B}}{\ominus}
$$

which are all compatible with the coaction of $C\left(\mathbb{G}_{2}\right)$. Furthermore, recall the unitaries

$$
f_{x}^{\varphi}: \mathcal{H}_{\varphi(x)} \rightarrow \mathcal{H}_{x} \underset{C\left(\mathbb{G}_{1}\right)}{\otimes} L^{2}(\mathcal{B}): \xi^{\varphi(x)} \mapsto X^{x}\left(\xi^{\varphi(x)} \otimes \Lambda\left(1_{\mathcal{B}}\right)\right)
$$

for $x \in \operatorname{Irred}\left(\mathbb{G}_{1}\right)$ of proposition 3.7. Note that these unitaries intertwine the representations of $\mathbb{G}_{2}$ on the two Hilbert spaces. We then also have

$$
f_{\varphi(x)}^{\varphi^{-1}}: \mathcal{H}_{x} \rightarrow \mathcal{H}_{\varphi(x)} \underset{C\left(\mathbb{G}_{2}\right)}{\bigotimes} L^{2}(\tilde{\mathcal{B}}): \eta^{x} \mapsto Z^{\varphi(x)}\left(\eta^{x} \otimes \tilde{\Lambda}\left(1_{\tilde{\mathcal{B}}}\right)\right)
$$

and combining them we have a unitary:

$\theta^{x}: \mathcal{H}_{x} \rightarrow \mathcal{H}_{x} \underset{C\left(\mathbb{G}_{1}\right)}{\otimes} L^{2}(\mathcal{B}) \underset{C\left(\mathbb{G}_{2}\right)}{\otimes} L^{2}(\tilde{\mathcal{B}}): \eta^{x} \mapsto X_{12}^{x} Z_{13}^{\varphi(x)}\left(\eta^{x} \otimes \Lambda\left(1_{\mathcal{B}}\right) \otimes \tilde{\Lambda}\left(1_{\tilde{\mathcal{B}}}\right)\right)$

Denoting by $X$ and $Z$ resp. $\oplus_{x \in \operatorname{Irred}\left(\mathbb{G}_{1}\right)} X^{x}$ and $\oplus_{x \in \operatorname{Irred}\left(\mathbb{G}_{1}\right)} Z^{\varphi(x)}$ (where we take the direct sum over the decomposition $\mathcal{H}=\bigoplus_{x \in \operatorname{Irred}\left(\mathbb{G}_{1}\right)} \mathcal{H}_{x}$ ), we then have a unitary

$$
\begin{aligned}
\theta & =\oplus_{x \in \operatorname{Irred}\left(\mathbb{G}_{1}\right)} \theta_{x}: \mathcal{H} \rightarrow \mathcal{H} \underset{C\left(\mathbb{G}_{1}\right)}{\otimes} L^{2}(\mathcal{B}) \underset{C\left(\mathbb{G}_{2}\right)}{\otimes} L^{2}(\tilde{\mathcal{B}}): \\
& \rightarrow X_{12} Z_{13}\left(\xi \otimes \Lambda\left(1_{\mathcal{B}}\right) \otimes \tilde{\Lambda}\left(1_{\tilde{\mathcal{B}}}\right)\right)
\end{aligned}
$$

and hence

$$
\begin{aligned}
& (\mathrm{id} \otimes \pi)\left(\alpha_{U}(a)\right) \theta(\xi)=(\mathrm{id} \otimes \pi)\left(U\left(a \otimes 1_{C\left(\mathbb{G}_{1}\right)}\right) U^{*}\right) X_{12} Z_{13}\left(\xi \otimes \Lambda\left(1_{\mathcal{B}}\right) \otimes \tilde{\Lambda}\left(1_{\tilde{\mathcal{B}}}\right)\right) \\
& \quad=X_{12} Z_{13}\left(a \otimes 1_{\mathcal{B}} \otimes 1_{\tilde{\mathcal{B}}}\right) Z_{13}^{*} X_{12}^{*} X_{12} Z_{13}\left(\xi \otimes \Lambda\left(1_{\mathcal{B}}\right) \otimes \tilde{\Lambda}\left(1_{\tilde{\mathcal{B}}}\right)\right) \\
& =X_{12} Z_{13}\left(a \xi \otimes \Lambda\left(1_{\mathcal{B}}\right) \otimes \tilde{\Lambda}\left(1_{\tilde{\mathcal{B}}}\right)\right)=\theta(a \xi),
\end{aligned}
$$

proving that $\theta(a \xi)=(\mathrm{id} \otimes \pi)\left(\alpha_{U}(a)\right)(\theta(\xi))$. This concludes the proof.

\section{Cocycle deformation of spectral triples}

In this section, we will fix a spectral triple $(\mathcal{A}, \mathcal{H}, D)$, a quantum group $\mathbb{G}$ acting algebraically on it by orientation-preserving isometries and a unitary fiber functor $\psi$ on $\mathbb{G}$, which satisfies $\operatorname{dim}\left(\mathcal{H}_{x}\right)=\operatorname{dim}\left(\mathcal{H}_{\psi(x)}\right)$ for every $x \in \operatorname{Irred}(\mathbb{G})$. Unitary fiber functors which satisfy this condition will be called dimension preserving and monoidal deformation via a dimension-preserving unitary fiber functor, a dimension-preserving monoidal deformation. Bichon et al. proved in [6] that dimension-preserving unitary fiber functors are in one-to-one correspondence with 2-cocycles on the dual quantum 
group. Using this, we will prove that dimension-preserving monoidal deformation is equivalent to the cocycle deformation introduced in [14]. In this section we will frequently use slight adaptations of the work of Bichon et al. [6].

\subsection{Cocycles on the dual of a compact quantum group}

Let $\mathbb{G}$ be a compact quantum group.

Definition $4.1^{2}$ Let $\mathbb{G}$ be a compact quantum group and $\left(c_{0}(\hat{\mathbb{G}}), \hat{\Delta}\right)$ its dual. We say a unitary element $\Omega \in \mathcal{M}\left(c_{0}(\hat{\mathbb{G}}) \otimes c_{0}(\hat{\mathbb{G}})\right)$ is a 2 -cocycle on $\hat{\mathbb{G}}$ if it satisfies

$$
(\Omega \otimes 1)(\hat{\Delta} \otimes \mathrm{id})(\Omega)=(1 \otimes \Omega)(\mathrm{id} \otimes \hat{\Delta})(\Omega) .
$$

Denoting for $x \in \operatorname{Irred}(\mathbb{G}), p_{x}$ to be the projection $c_{0}(\hat{\mathbb{G}}) \rightarrow B\left(\mathcal{H}_{x}\right)$, we will say a cocycle is normalized if $\left(p_{\varepsilon} \otimes \mathrm{id}\right) \Omega=p_{\varepsilon} \otimes \mathrm{id}$ and $\left(\mathrm{id} \otimes p_{\varepsilon}\right) \Omega=\mathrm{id} \otimes p_{\varepsilon}$. From now on, we will always assume 2-cocycles to be normalized.

Proposition 4.2 ([6]) Let $\Omega$ be a normalized unitary 2-cocycle on $\hat{\mathbb{G}}$ and denote

$$
\Omega_{(2)}=(\Omega \otimes 1)(\hat{\Delta} \otimes \mathrm{id})(\Omega)=(1 \otimes \Omega)(\mathrm{id} \otimes \hat{\Delta})(\Omega) .
$$

Then there exists a unique unitary fiber functor $\psi_{\Omega}$ on $\mathbb{G}$ such that

$$
\mathcal{H}_{\psi_{\Omega}(x)}=\mathcal{H}_{x}, \quad \psi_{\Omega}(S)=\Omega S, \quad \psi_{\Omega}(T)=\Omega_{(2)} T
$$

for all $S \in \operatorname{Mor}(y \otimes z, x)$ and $T \in \operatorname{Mor}(x \otimes y \otimes z, a)$ and $x, y, z \in \operatorname{Irred}(\mathbb{G})$. Moreover, it is dimension preserving.

Proof The proof follows directly as our $\psi$ satisfies the conditions of Remark 2.11. That it is dimension preserving follows directly by construction.

Using this unitary fiber functor, one can make a new compact quantum group $\mathbb{G}_{\Omega}=\left(C\left(\mathbb{G}_{\Omega}\right), \Delta_{\Omega}\right)[6]$ and a monoidal equivalence $\varphi: \mathbb{G} \rightarrow \mathbb{G}_{\Omega}$ along the lines of Proposition 2.12. Note that the dual quantum group will be $\left(c_{0}\left(\hat{\mathbb{G}}_{\Omega}\right), \hat{\Delta}_{\Omega}\right)$, where

$$
c_{0}\left(\hat{\mathbb{G}}_{\Omega}\right)=\bigoplus_{x \in \operatorname{Irred}(\mathbb{G})} B\left(\mathcal{H}_{x}\right)=c_{0}(\hat{\mathbb{G}})
$$

and

$$
\hat{\Delta}_{\Omega}(a) \psi_{\Omega}(S)=\psi_{\Omega}(S) a
$$

in which $\hat{\Delta}_{\Omega}(a)=\Omega \Delta(a) \Omega^{*}$.

\footnotetext{
${ }^{2}$ In [6], the authors use another convention for cocycle. In fact, if $\Omega$ is a cocycle in our sense, $\Omega^{*}$ is one in the sense of Bichon and coauthors.
} 
Proposition 4.3 ([6]) For every dimension-preserving unitary fiber functor $\psi$ on a quantum group $\mathbb{G}$, there exists a normalized unitary 2-cocycle $\Omega$ on $\hat{\mathbb{G}}$ such that $\psi \cong \psi_{\Omega}$.

Proof The proof is a slightly adapted version of the proof of proposition 4.5 in [6].

This theorem tells us that every dimension-preserving monoidal equivalence comes from a cocycle. The next step to prove that a dimension-preserving monoidal deformation of a spectral triple is a cocycle deformation is to introduce the algebraic notion of a 2-cocycle. We will prove that every 2-cocycle on the dual of a compact quantum group induces an algebraic 2-cocycle on the compact quantum group and that the monoidal deformation is equivalent to a cocycle deformation of the spectral triple as was introduced by Goswami in [14].

\subsection{Algebraic 2-cocycle deformation of a spectral triple}

We will start with defining the algebraic counterpart of a 2-cocycle on the dual of a compact quantum group. In algebraic literature (for example Schauenburg [19]), the definition and theorems are stated for Hopf algebras. We make slight adaptations to Hopf *-algebras.

Definition 4.4 Let $H$ be a Hopf algebra.

1. An (algebraic) dual 2-cocycle on $H$ is a linear map $\sigma: H \odot H \rightarrow \mathbb{C}$, such that

$$
\sigma\left(a_{(1)}, b_{(1)}\right) \sigma\left(a_{(2)} b_{(2)}, c\right)=\sigma\left(b_{(1)}, c_{(1)}\right) \sigma\left(a, b_{(2)} c_{(2)}\right)
$$

for all $a, b, c \in H$. Moreover, a dual 2-cocycle is called normalized if $\sigma(1, h)=$ $\sigma(h, 1)=\varepsilon(h)$ for all $h \in H$.

2. A dual 2-cocycle is called invertible if there exists a linear map $\sigma^{\prime}: H \odot H \rightarrow \mathbb{C}$ such that

$$
\sigma\left(a_{(1)}, b_{(1)}\right) \sigma^{\prime}\left(a_{(2)}, b_{(2)}\right)=\varepsilon(a) \varepsilon(b)=\sigma^{\prime}\left(a_{(1)}, b_{(1)}\right) \sigma\left(a_{(2)}, b_{(2)}\right)
$$

In this case, $\sigma^{\prime}$ is called the inverse dual cocycle and written as $\sigma^{-1}$. Moreover, $\sigma^{-1}$ satisfies

$$
\sigma^{-1}\left(a_{(1)} b_{(1)}, c\right) \sigma^{-1}\left(a_{(2)}, b_{(2)}\right)=\sigma^{-1}\left(a, b_{(1)} c_{(1)}\right) \sigma^{-1}\left(b_{(2)}, c_{(2)}\right) .
$$

3. If $H$ is a Hopf*-algebra, a dual 2-cocycle $\sigma$ is called unitary if it satisfies

$$
\overline{\sigma(a, b)}=\sigma^{-1}\left(S(a)^{*}, S(b)^{*}\right) .
$$

In that case, we also have

$$
\overline{\sigma^{-1}(a, b)}=\sigma\left(S(a)^{*}, S(b)^{*}\right) .
$$


In the rest of the section, when we use 2-cocycles on Hopf*-algebras, we will always assume them to be unitary.

Using such a dual 2-cocycle, we can make a new *-algebra and several new $H$ comodule ${ }^{*}$-algebras. We will use the following linear maps:

- $U: H \rightarrow \mathbb{C}: h \mapsto \sigma\left(h_{(1)}, S\left(h_{(2)}\right)\right)$,

- $V: H \rightarrow \mathbb{C}: h \mapsto U\left(S^{-1}(h)\right)$.

One can prove that for $U^{-1}(h)=\sigma^{-1}\left(S\left(h_{(1)}\right), h_{(2)}\right)$ and $V^{-1}(h)=U^{-1}\left(S^{-1}(h)\right)$, one has $U\left(h_{(1)}\right) U^{-1}\left(h_{(2)}\right)=\varepsilon(h)=U^{-1}\left(h_{(1)}\right) U\left(h_{(2)}\right)$ and $V\left(h_{(1)}\right) V^{-1}\left(h_{(2)}\right)=$ $\varepsilon(h)=V^{-1}\left(h_{(1)}\right) V\left(h_{(2)}\right)$.

Definition 4.5 Given an invertible dual 2-cocycle $\sigma$ on a Hopf *-algebra $\left(H, \Delta, \varepsilon, S,{ }^{*}\right)$, we define $\left(H^{\sigma}, \Delta_{\sigma}, \varepsilon_{\sigma}, S_{\sigma},{ }^{*} \sigma\right)$ to be the twisted Hopf ${ }^{*}$-algebra, which

- is isomorphic to $H$ as a co-algebra,

- has multiplication defined by $g \cdot \sigma h=\sigma\left(g_{(1)}, h_{(1)}\right) g_{(2)} h_{(2)} \sigma^{-1}\left(g_{(3)}, h_{(3)}\right)$,

- has antipode $S_{\sigma}(h)=U\left(h_{(1)}\right) S\left(h_{(2)}\right) U^{-1}\left(h_{(3)}\right)$,

- has counit $\varepsilon_{\sigma}=\varepsilon$

- and has involution $h^{* \sigma}=V^{-1}\left(h_{(1)}^{*}\right) h_{(2)}^{*} V\left(h_{(3)}^{*}\right)$.

Definition 4.6 We define

1. $\mathbb{C}_{\sigma} H$ to be a $H^{\sigma}-H$-bicomodule ${ }^{*}$-algebra which

- is isomorphic to $H$ as right $H$-comodule,

- has twisted multiplication $(1 \# g)(1 \# h)=\sigma\left(g_{(1)}, h_{(1)}\right) \# g_{(2)} h_{(2)}$,

- has a coaction $\beta_{1}: \mathbb{C}_{\sigma} H \rightarrow H^{\sigma} \odot\left(\mathbb{C}_{\sigma} H\right):(1 \# h) \mapsto h_{(1)} \otimes\left(1 \# h_{(2)}\right)$,

- and has involution $(1 \# h)^{* \mathbb{C}_{\sigma} H}=1 \# V^{-1}\left(h_{(1)}^{*}\right) h_{(2)}^{*}$

and

2. $H_{\sigma^{-1}} \# \mathbb{C}$ to be a $H-H^{\sigma}$-bicomodule algebra which

- is isomorphic to $H$ as left $H$-comodule,

- has twisted multiplication $(g \# 1)(h \# 1)=g_{(1)} h_{(1)} \# \sigma^{-1}\left(g_{(2)}, h_{(2)}\right)$,

- has a coaction $\beta_{2}: H_{\sigma^{-1}} \# \mathbb{C} \rightarrow\left(H_{\sigma^{-1}} \# \mathbb{C}\right) \odot H^{\sigma}:(h \# 1) \mapsto\left(h_{(1)} \# 1\right) \otimes h_{(2)}$,

- and has involution $(h \# 1)^{* H_{\sigma^{-1}} \# \mathbb{C}}=h_{(1)}^{*} V\left(h_{(2)}^{*}\right) \# 1$.

Definition 4.7 Let $H$ be a Hopf*-algebra and $\sigma$ an invertible dual 2-cocycle on $H$. Let $A$ be a right $H$-comodule ${ }^{*}$-algebra with coaction $\alpha: A \rightarrow A \odot H$. We define $A_{\sigma^{-1}} \# \mathbb{C}$ to be a right $H^{\sigma}$-comodule ${ }^{*}$-algebra which

- is isomorphic to $A$ as vector space,

- has multiplication $(a \# 1)\left(a^{\prime} \# 1\right)=a_{(0)} a_{(0)}^{\prime} \# \sigma^{-1}\left(a_{(1)}, b_{(1)}\right)$,

- has a coaction $\tilde{\alpha}: A_{\sigma^{-1}} \# \mathbb{C} \rightarrow\left(A_{\sigma^{-1}} \# \mathbb{C}\right) \odot H^{\sigma}:(a \# 1) \mapsto\left(a_{(0)} \# 1\right) \otimes a_{(1)}$,

- and has involution $(a \# 1)^{* \mathbb{C} \# \sigma H}=a_{(0)} V\left(a_{(1)}^{*}\right) \# 1$.

Theorem 4.8 Let $H$ be a Hopf ${ }^{*}$-algebra and $A$ a right $H$-comodule*-algebra with coaction $\alpha: A \rightarrow A \odot H$. Denote $B=H_{\sigma^{-1}} \# \mathbb{C}$. Then,

$$
A \underset{H}{\square} B \cong A_{\sigma^{-1}} \# \mathbb{C} .
$$


Proof We have the natural *-algebraic isomorphisms

$$
A \stackrel{\alpha}{\rightarrow} A \underset{H}{\bullet} H \stackrel{\text { id } \otimes \varepsilon}{\rightarrow} A
$$

Using it as vector space isomorphisms, deforming the multiplications and using that $B$ and $H$ are isomorphic as left $H$-comodules, it is easy to check that we have a well-defined *-algebra isomorphism

$$
\lambda: A_{\sigma^{-1}} \# \mathbb{C} \rightarrow A \underset{H}{\bullet} B:(a \# 1) \rightarrow a_{(0)} \otimes\left(a_{(1)} \# 1\right) .
$$

In this paragraph, we give a slightly adapted version of a result of Goswami and Joardar in [14].

Theorem $4.9\left([14]^{3}\right)$ Let $(\mathcal{A}, \mathcal{H}, D)$ be a spectral triple and $\mathbb{G}$ a compact quantum group acting on it algebraically and by orientation-preserving isometries with the representation $U$. Let $\sigma$ be an (algebraic) unitary dual 2-cocycle on $\mathcal{O}(\mathbb{G})$. Then,

(a) there exists a representation $\pi_{\sigma}: \mathcal{A}_{\sigma^{-1}} \# \mathbb{C} \rightarrow B(\mathcal{H})$,

(b) $\left(\mathcal{A}_{\sigma^{-1}} \# \mathbb{C}, \mathcal{H}, D\right)$ is a spectral triple.

Proof (a) Denote the coaction $\alpha=\operatorname{ad}_{U}$ of $\mathcal{O}(\mathbb{G})$ on $\mathcal{A}_{\sigma^{-1}} \# \mathbb{C}$ by $\alpha(a)=a_{(0)} \otimes a_{(1)}$. Let $\mathcal{N}$ be a dense subspace of $\mathcal{H}$ such that $U(\mathcal{N}) \subset \mathcal{N} \odot \mathcal{O}(\mathbb{G})$, and on that subspace, let $U(\xi)=\xi_{(0)} \otimes \xi_{(1)}$. Then we can define for $a \in \mathcal{A}_{\sigma^{-1}} \# \mathbb{C}$ :

$$
\pi_{\sigma}(a): \mathcal{H} \rightarrow \mathcal{H}: \xi \mapsto a_{(0)} \xi_{(0)} \sigma^{-1}\left(a_{(1)}, \xi_{(1)}\right) .
$$

In section 4.3 of [14], it is proved that $\pi_{\sigma}(a)$ is bounded for all $a \in \mathcal{A}_{\sigma^{-1}} \# \mathbb{C}$ and that $\pi_{\sigma}$ is a well-defined ${ }^{*}$-morphism.

(b) This is theorem 4.10(4) in [14].

\subsection{Linking dimension-preserving monoidal equivalences with algebraic cocycles}

In Proposition 4.3, we proved that there is an equivalence between dimensionpreserving unitary fiber functors on a compact quantum group $\mathbb{G}$ and cocycles on the dual $\mathbb{G}$. In the following Theorem 4.10 , we will prove that there is also an equivalence between cocycles on $\hat{\mathbb{G}}$ and (algebraic) dual cocycles on $\mathcal{O}(\mathbb{G})$. Moreover, we will show in Theorem 4.11 that the bi-Galois object $\mathcal{B}$ associated with the monoidal equivalence induced by the fiber functor will be of the form $\mathcal{B}=\mathcal{O}(\mathbb{G})_{\sigma^{-1}} \# \mathbb{C}$.

\footnotetext{
3 We want to note that Goswami erroneously referred to [16] to explain the deformation of the Hopf *algebra. Indeed, Majid uses a reality condition and Goswami a unitarity condition, which makes the theory of Majid not applicable here. We developed a new deformation of the star structure using a unitary cocycle which results in Definitions 4.5 and 4.6.
} 
Theorem 4.10 Let $\mathbb{G}$ be a compact quantum group. If $\Omega$ is a unitary 2-cocycle on the dual $\hat{\mathbb{G}}$, the formula

$$
\sigma\left(u_{i j}^{x} \otimes u_{k l}^{y}\right)=\left\langle\xi_{i}^{x} \otimes \xi_{k}^{y}, \Omega\left(\xi_{j}^{x} \otimes \xi_{l}^{y}\right)\right\rangle, \quad x, y \in \operatorname{Irred}(\mathbb{G})
$$

defines a unique (algebraic) unitary dual 2-cocycle $\sigma$ on $\mathcal{O}(\mathbb{G})$. On the other hand, if $\sigma$ is an (algebraic) unitary dual 2-cocycle on $\mathcal{O}(\mathbb{G})$, formula 4.2 uniquely defines a unitary 2-cocycle $\Omega$ on $\hat{\mathbb{G}}$.

Proof Under the first assumption, as the $u_{i j}^{x}$ constitute a basis of $\mathcal{O}(\mathbb{G})$, the bilinear map $\sigma$ is well defined. Using the cocycle property (4.1) of $\Omega$, one can check that $\sigma$ satisfies the dual cocycle condition in Definition 4.4(1). It is normalized and unitary as $\Omega$ is normalized and unitary. Under the second assumption, $\Omega$ is uniquely and well defined as element of $\mathcal{M}\left(c_{0}(\hat{\mathbb{G}}) \otimes c_{0}(\hat{\mathbb{G}})\right)$. The dual cocycle condition in Definition 4.4(1) will imply the cocycle condition (4.1) of $\Omega$. Again, $\Omega$ is normalized and unitary as $\sigma$ is.

Remark that, as $\Omega^{*}$ is the inverse of $\Omega$, we see that $\sigma^{\prime}$ associated with $\Omega^{*}$ is the convolution inverse of $\sigma$. We will denote it with $\sigma^{-1}$ and we have

$$
\sigma^{-1}\left(u_{i j}^{x} \otimes u_{k l}^{y}\right)=\left\langle\xi_{i}^{x} \otimes \xi_{k}^{y}, \Omega^{*}\left(\xi_{j}^{x} \otimes \xi_{l}^{y}\right)\right\rangle
$$

Theorem 4.11 Let $\mathbb{G}$ be a compact quantum group with a dimension-preserving unitary fiber functor $\psi$. Let $\mathcal{B}$ be the bi-Galois object associated with $\psi$ with coaction $\beta_{1}: \mathcal{B} \rightarrow \mathcal{O}\left(\mathbb{G}_{1}\right) \odot \mathcal{B} ;$ let $\Omega$ be the unitary 2-cocycle on the dual $\hat{\mathbb{G}}$ associated with $\psi \cong \psi_{\Omega}$ and $\sigma$ the algebraic dual 2-cocycle equivalent to $\Omega$ (Proposition 4.10). Then there exists $a^{*}$-algebra isomorphism

$$
\chi: \mathcal{B} \rightarrow \mathcal{O}(\mathbb{G})_{\sigma^{-1}} \# \mathbb{C}
$$

such that $(\mathrm{id} \otimes \chi) \beta_{1}=\Delta \circ \chi$.

Proof Denoting $\varphi: \mathbb{G} \rightarrow \mathbb{G}_{\Omega}$ to be the monoidal equivalence associated with $\psi$, we can find unitaries $u_{x}=\mathcal{H}_{x} \rightarrow \mathcal{H}_{\varphi(x)}$, as $\operatorname{dim}(\varphi(x))=\operatorname{dim}(x)$ for all $x \in \operatorname{Irred}(\mathbb{G})$. Fixing a $x \in \operatorname{Irred}(\mathbb{G})$, we can define $Y^{x}=X^{x}\left(u_{x} \otimes 1\right) \in B\left(\mathcal{H}_{x}\right) \odot \mathcal{B}$ and

$$
Y^{\prime}=\oplus_{x \in \operatorname{Irred}(\mathbb{G})} Y^{x} \in \mathcal{M}\left(c_{0}(\hat{\mathbb{G}}) \otimes B_{r}\right)
$$

(where we take the direct sum over all classes, all of them with multiplicity one). Note that the matrix coefficients of the $X^{x}$ constitute a basis of $\mathcal{B}$ by Theorem 2.13. As the $u_{x}$ are unitaries, also the matrix coefficients of the $Y^{x}$ (let us call them $b_{i j}^{x}$ ) and hence of $Y^{\prime}$ form a basis of $\mathcal{B}$. As both the $\left(u_{i j}^{x}\right)_{i j, x}$ and $\left(b_{i j}^{x}\right)_{i j, x}$ are bases of $\mathcal{O}(\mathbb{G})$ resp. $\mathcal{B}$, we have a vector space isomorphism

$$
\chi: \mathcal{O}(\mathbb{G}) \rightarrow \mathcal{B}: u_{i j}^{x} \mapsto b_{i j}^{x},
$$


which is compatible with the coactions (i.e. (id $\left.\otimes \chi) \Delta=\beta_{1} \circ \chi\right)$. Moreover, one can prove that, analogously as in the proof proposition 4.5 of [6], $Y^{\prime}$ satisfies the equation

$$
(\hat{\Delta} \otimes \mathrm{id})\left(Y^{\prime}\right)=Y_{13}^{\prime} Y_{23}^{\prime}\left(\Omega \otimes 1_{\mathcal{B}}\right)
$$

As $(\hat{\Delta} \otimes \mathrm{id})\left(Y^{\prime}\right)=(\hat{\Delta} \otimes \chi)(\mathbb{V})$ by construction and $(\hat{\Delta} \otimes \mathrm{id})(\mathbb{V})=\mathbb{V}_{13} \mathbb{V}_{23}$ by definition of $\mathbb{V}$, one can prove that

$$
\begin{aligned}
\chi\left(u_{i j}^{x} u_{s t}^{y}\right) & =\left\langle\xi_{i}^{x} \otimes \xi_{s}^{y} \otimes \mathrm{id},(\hat{\Delta} \otimes \mathrm{id})\left(Y^{\prime}\right)\left(\xi_{j}^{x} \otimes \xi_{t}^{y} \otimes \mathrm{id}\right)\right\rangle \\
& =\left\langle\xi_{i}^{x} \otimes \xi_{s}^{y} \otimes \mathrm{id},\left(Y_{13}^{\prime} Y_{23}^{\prime}(\Omega \otimes \mathrm{id})\right)\left(\xi_{j}^{x} \otimes \xi_{t}^{y} \otimes \mathrm{id}\right)\right\rangle \\
& =\sum_{p, q} \chi\left(u_{i p}^{x}\right) \chi\left(u_{s q}^{y}\right) \sigma\left(u_{p j}^{x}, u_{q t}^{y}\right),
\end{aligned}
$$

where we used Theorem 4.10. Hence, also $\chi\left(u_{i j}^{x}\right) \chi\left(u_{s t}^{y}\right)=\sum_{k, l} \chi\left(u_{i k}^{x} u_{s l}^{y}\right) \sigma^{-1}$ $\left(u_{k j}^{x}, u_{l t}^{y}\right)$, which means

$$
\chi(a) \chi(b)=\chi\left(a_{(0)} b_{(0)}\right) \sigma^{-1}\left(a_{(1)}, b_{(1)}\right) .
$$

Finally, to check that $\chi$ is a ${ }^{*}$-algebra isomorphism, note that by the previous equation, we also have

$$
\chi\left(a b^{*}\right)=\chi\left(a_{(0)}\right) \chi\left(b_{(0)}^{*}\right) \sigma\left(a_{(1)}, b_{(1)}^{*}\right),
$$

and hence

$$
\begin{aligned}
\chi\left(u_{i j}^{x}\right)^{*} & =\sum_{k, l} \chi\left(u_{k j}^{x}\right)^{*} \chi\left(u_{k l}^{x}\left(u_{i l}^{x}\right)^{*}\right)=\sum_{k, l, p, q} \chi\left(u_{k j}^{x}\right)^{*} \chi\left(u_{k p}^{x}\right) \chi\left(\left(u_{i q}^{x}\right)^{*}\right) \sigma\left(u_{p l}^{x},\left(u_{q l}^{x}\right)^{*}\right) \\
& =\sum_{l, q} \chi\left(\left(u_{i q}^{x}\right)^{*}\right) \sigma\left(u_{j l}^{x},\left(u_{q l}^{x}\right)^{*}\right)
\end{aligned}
$$

by unitarity of the $U^{x}$ and the $Y^{x}$, which implies that

$$
\chi(a)^{*}=\chi\left(a_{(1)}^{*}\right) \sigma\left(S\left(a_{(3)}\right)^{*}, a_{(2)}^{*}\right)=\chi\left(a_{(1)}^{*}\right) V\left(a_{(2)}^{*}\right)
$$

where $V(a)=\sigma\left(S^{-1}\left(a_{(2)}\right), a_{(1)}\right)$ as before. This proves the last statement.

\subsection{Dimension-preserving monoidal deformation is isomorphic to algebraic 2-cocycle deformation}

In this last paragraph of Sect. 4, we state and prove the main result of this section: the Goswami-Joardar cocycle deformation amounts to our deformation with a dimensionpreserving monoidal equivalence. 
Theorem 4.12 Let $(\mathcal{A}, \mathcal{H}, D)$ be a spectral triple, $\mathbb{G}$ a compact quantum group acting on it algebraically and by orientation-preserving isometries via a unitary representation $U$ and let $\psi$ be a dimension-preserving unitary fiber functor on $\mathbb{G}$. Denoting by $\mathcal{B}$ the corresponding bi-Galois object, there exists an (algebraic) unitary dual 2-cocycle $\sigma$ such that $\left(\mathcal{A} \underset{\mathcal{O}(\mathbb{G})}{\square} \mathcal{B}, \mathcal{H} \underset{C(\mathbb{G})}{\otimes} L^{2}(\mathcal{B}), \tilde{D}\right)$ defined in Sect. 3 and $\left(\mathcal{A}_{\sigma^{-1}} \# \mathbb{C}, \mathcal{H}, D\right)$ are isomorphic as spectral triples.

Remember that $\mathcal{B}$ is the bi-Galois object associated with the fiber functor $\psi, L^{2}(\mathcal{B})$ the GNS-space with respect to the invariant state $\omega=(h \otimes \mathrm{id}) \beta_{1}$ and the deformed Dirac operator $\tilde{D}$ from Sect. 3 . We give the proof via some propositions.

Proposition 4.13 1. There exists a unitary $Y \in \mathcal{M}\left(\mathcal{K}(\mathcal{H}) \otimes B_{r}\right)$ such that $\phi: \mathcal{H} \rightarrow$ $\mathcal{H} \otimes L^{2}(\mathcal{B}): \xi \rightarrow Y(\xi \otimes 1)$ is an isomorphism of Hilbert spaces. $C(\mathbb{G})$

2. Under this isomorphism, $\phi D=\tilde{D} \phi$.

3. $\mathcal{A}_{\mathcal{O}(\mathbb{G})}^{\square_{1}} \mathcal{B} \cong \mathcal{A}_{\sigma^{-1}} \# \mathbb{C}$ with $\sigma$ the algebraic dual 2-cocycle associated with the dimension-preserving unitary fiber functor $\psi$.

Proof 1. Recall the unitaries $u_{x}: \mathcal{H}_{x} \rightarrow \mathcal{H}_{\varphi(x)}$ from the proof of Theorem 4.11 and the mutually inverse unitaries

$$
f_{x}: \mathcal{H}_{\varphi(x)} \rightarrow \mathcal{H}_{x} \underset{C(\mathbb{G})}{\otimes} L^{2}(\mathcal{B}): \xi \mapsto X^{x}\left(\xi \otimes \Lambda\left(1_{\mathcal{B}}\right)\right)
$$

and

$$
g_{x}: \mathcal{H}_{x} \underset{C(\mathbb{G})}{\otimes} L^{2}(\mathcal{B}) \rightarrow \mathcal{H}_{\varphi(x)}: z \mapsto\left(\operatorname{id}_{\mathcal{H}_{\varphi(x)}} \otimes \omega_{1}^{\prime}\right)\left(X^{x *} z\right)
$$

from the proof of Proposition 3.7 point 1. Therefore, defining $\phi_{x}=f_{x} \circ u_{x}=$ : $\mathcal{H}_{x} \rightarrow \mathcal{H}_{x} \underset{C(\mathbb{G})}{\otimes} L^{2}(\mathcal{B}), \phi_{x}^{\prime}=u_{x}^{*} \circ g_{x}: \mathcal{H}_{x} \underset{C(\mathbb{G})}{\otimes} L^{2}(\mathcal{B}) \rightarrow \mathcal{H}_{x}$ and $Y=$ $\oplus_{x \in \operatorname{Irred}(\mathbb{G})} Y^{x}$, we can make $\phi=\sum_{x \in \operatorname{Irred}(\mathbb{G})} \phi_{x}$ (where in both cases we take the sum over the irreducible representations appearing in the decomposition of $U$ ) such that $\phi(\xi)=Y(\xi \otimes 1)$ for $\xi \in \mathcal{H} . Y$ is unitary and hence $\phi$ is the desired isomorphism of Hilbert spaces.

2. We have to prove that $\phi(D \xi)=\tilde{D}(\phi(\xi))$ for $\xi \in \operatorname{dom}(D)$. Denoting $P_{\lambda_{n}}$ resp. $\tilde{P}_{\lambda_{n}}$ to be the projection onto $V_{\lambda_{n}}$ resp. $V_{\lambda_{n}} \underset{C(\mathbb{G})}{\bigotimes} L^{2}(\mathcal{B})$, note that, as $Y=(\mathrm{id} \otimes \chi)(U)$ and $U$ commutes with $D, \phi\left(P_{\lambda_{n}} \xi\right)=\tilde{P}_{\lambda_{n}}(\phi(\xi))$. Then,

$$
\sum_{n}\left|\lambda_{n}\right|^{2}\left\|\tilde{P}_{\lambda_{n}}(\phi(\xi))\right\|^{2}=\sum_{n}\left|\lambda_{n}\right|^{2}\left\|\phi\left(P_{\lambda_{n}}(\xi)\right)\right\|^{2}=\sum_{n}\left|\lambda_{n}\right|^{2}\left\|P_{\lambda_{n}}(\xi)\right\|^{2}<\infty
$$

as $\xi \in \operatorname{dom}(D)$ and hence $\phi$ maps the domain of $D$ into the domain of $\tilde{D}$. Also, by the previous remark, trivially, $\tilde{D}_{n}=\tilde{D}_{\left.\right|_{V_{\lambda_{n}}} \underset{C(\mathbb{G})}{\otimes L^{2}(\mathcal{B})}}$ commutes with $\phi$ for all $n$.

Taking the direct sum, we can conclude that also $\tilde{D}$ commutes with $\phi$. 
3. The proof follows from Theorems 4.8 and 4.11 .

Finally, it suffices to prove that the actions of the algebras on the Hilbert spaces are isomorphic.

Proposition 4.14 The action of $\mathcal{A}_{\sigma^{-1}} \# \mathbb{C}$ on $\mathcal{H}$ is isomorphic to the action of $\mathcal{A} \underset{\mathcal{O}(\mathbb{G})}{\square} \mathcal{B}$ on $\mathcal{H} \underset{C(\mathbb{G})}{\otimes} L^{2}(\mathcal{B})$, i.e. if $\phi: \mathcal{H} \rightarrow \mathcal{H} \underset{C(\mathbb{G})}{\otimes} L^{2}(\mathcal{B})$ and $(\mathrm{id} \otimes \chi) \alpha: \mathcal{A}_{\sigma^{-1}} \# \mathbb{C} \rightarrow$ $\mathcal{A} \square \mathcal{B}$ are the isomorphisms of the previous proposition, we have:

$$
\phi(a \cdot \sigma \xi)=(\mathrm{id} \otimes \chi) \alpha(a) \phi(\xi) .
$$

Proof Let $a \in \mathcal{A}$ and $\xi_{n}^{z, m}$ be the $n$-th basisvector in the $m$-th summand of $\mathcal{H}_{z}$ in the decomposition of $\mathcal{H}$. Using the Hilbert space isomorphism $\phi: \mathcal{H} \rightarrow \mathcal{H} \underset{C(\mathbb{G})}{\otimes} L^{2}(\mathcal{B})$ and the notation $a \cdot{ }_{\sigma} \xi_{n}^{z, m}$ for the deformed action of $a \# 1 \in \mathcal{A}_{\sigma^{-1}} \# \mathbb{C}$ on $\xi$, we will prove that

$$
\phi(a \cdot \sigma \xi)=(\operatorname{id} \otimes \chi) \alpha(a) \phi(\xi)
$$

by proving

$$
a \cdot \sigma \xi=Y^{*}(\mathrm{id} \otimes \chi) \alpha(a)(Y(\xi \otimes 1))
$$

First, we compute $a \cdot \sigma \xi_{n}^{z, m}$. Writing

$$
U\left(\xi_{j}^{x, k} \otimes \mathrm{id}\right)=\sum_{i} \xi_{i}^{x, k} \otimes u_{i j}^{x, k}
$$

and noting that $\alpha_{U}(a)=U(a \otimes 1) U^{*}$ and that $a \cdot \sigma \xi=a_{(0)} \xi_{(0)} \sigma^{-1}\left(a_{(1)}, \xi_{(1)}\right)$ where $U(\xi \otimes 1)=\xi_{(0)} \otimes \xi_{(1)}$, we only need a calculation to check that

$$
a \cdot \sigma \xi_{n}^{z, m}=\sum_{x, k, i, j, q} \xi_{i}^{x, k}\left\langle\xi_{j}^{x, k}, a \xi_{q}^{z, m}\right\rangle \sum_{s} \sigma^{-1}\left(u_{i j}^{x, k}\left(u_{s q}^{z, m}\right)^{*}, u_{s n}^{z, m}\right),
$$

which is a finite sum as $\left(\alpha_{U}\right)_{\left.\right|_{\mathcal{A}}}$ is an algebraic coaction. Using, moreover, the cocycle relations, we get

$$
a \cdot \sigma \xi_{n}^{z, m}=\sum_{x, k, i, j, q} \xi_{i}^{x, k}\left\langle\xi_{j}^{x, k}, a \xi_{q}^{z, m}\right\rangle \sum_{t, r} \sigma^{-1}\left(\left(u_{t r}^{z, m}\right)^{*}, u_{t n}^{z, m}\right) \sigma\left(u_{i j}^{x, k},\left(u_{r q}^{z, m}\right)^{*}\right)
$$

Next, we will compute $Y^{*}(\mathrm{id} \otimes \chi) \alpha_{U}(a) Y\left(\xi_{n}^{z, m} \otimes 1\right)$. Writing $Y\left(\xi_{j}^{x, k} \otimes\right.$ id $)=$ $\sum_{i} \xi_{i}^{x, k} \otimes \chi\left(u_{i j}^{x, k}\right)$, we have

$$
\begin{aligned}
Y^{*}(\mathrm{id} \otimes \chi) \alpha_{U}(a) Y\left(\xi_{n}^{z, m} \otimes 1\right)= & \sum_{x, k, i, j, q} \xi_{i}^{x, k}\left\langle\xi_{j}^{x, k}, a \xi_{q}^{z, m}\right\rangle \\
& \otimes \sum_{s, t}\left(\chi\left(u_{s i}^{x, k}\right)\right)^{*} \chi\left(u_{s j}^{x, k}\left(u_{t q}^{z, m}\right)^{*}\right) \chi\left(u_{t n}^{z, m}\right) .
\end{aligned}
$$


Note now that by Eq. (4.4),

$$
\chi\left(u_{s j}^{x, k}\left(u_{t q}^{z, m}\right)^{*}\right)=\chi\left(u_{s v}^{x, k}\right) \chi\left(\left(u_{t r}^{z, m}\right)^{*}\right) \sigma\left(u_{v j}^{x},\left(u_{r q}^{z}\right)^{*}\right)
$$

and by unitarity of the $u_{i j}^{x}$ and the $\chi\left(u_{i j}^{x}\right)$ and Theorem 4.11, we get

$$
\sum_{s, t}\left(\chi\left(u_{s i}^{x, k}\right)\right)^{*} \chi\left(u_{s j}^{x, k}\left(u_{t q}^{z, m}\right)^{*}\right) \chi\left(u_{t n}^{z, m}\right)=\sum_{t, r} \sigma^{-1}\left(\left(u_{t r}^{z, m}\right)^{*}, u_{t n}^{z, m}\right) \sigma\left(u_{i j}^{x, k},\left(u_{r q}^{z, m}\right)^{*}\right),
$$

which implies that

$$
\begin{aligned}
Y^{*}(\mathrm{id} \otimes \chi) \alpha_{U}(a) Y\left(\xi_{n}^{z, m} \otimes 1\right)= & \sum_{x, k, i, j, q} \xi_{i}^{x, k}\left\langle\xi_{j}^{x, k}, a \xi_{q}^{z, m}\right\rangle \\
& \sum_{t, r} \sigma^{-1}\left(\left(u_{t r}^{z, m}\right)^{*}, u_{t n}^{z, m}\right) \sigma\left(u_{i j}^{x, k},\left(u_{r q}^{z, m}\right)^{*}\right) .
\end{aligned}
$$

We can conclude that

$$
\phi(a \cdot \sigma \xi)=(\operatorname{id} \otimes \chi) \alpha(a) \phi(\xi)
$$

and with this, the proof of Theorem 4.12 is completed.

\section{Constructing a non-dimension-preserving example}

In this section, we will construct an example of a monoidal deformation coming from a non-dimension-preserving monoidal equivalence. We will use the spectral triple on the Podleś spheres [17] defined in [10] and $S U_{q}(2)$, which acts on it in the appropriate way.

\subsection{Monoidal equivalences on $S U_{q}(2)$}

We look at orthogonal quantum groups and $S U_{q}(2)$ in particular.

Definition 5.1 ([21]) Let $n \in \mathbb{N}$ and $F \in \operatorname{GL}(n, \mathbb{C})$ with $F \bar{F}=c I_{n} \in \mathbb{R} I_{n}$. Then, $A_{o}(F)$ is defined as the universal quantum group generated by the coefficients of the matrix $U \in M_{n}\left(A_{o}(F)\right)$ with relations

- $U$ is a unitary and

- $U=F \bar{U} F^{-1}$.

Moreover, $A_{o}(F)=\left(C\left(A_{o}(F)\right), U\right)$ is a compact matrix quantum group (as defined in [24]). They are called universal orthogonal quantum groups. 
As the matrices $F$ are not in one to one correspondence with the universal quantum groups (i.e. different matrices $F$ can define the same universal quantum groups), it is necessary (but not so hard) to classify the quantum groups $A_{o}(F)$. This has been done in [6].

Proposition 5.2 For $F_{1}, F_{2}$ matrices in $\operatorname{GL}(n, \mathbb{C})$ with $F_{i} \bar{F}_{i}= \pm 1$, we say

$$
F_{1} \sim F_{2} \text { if there exists a unitary } v \in U(n) \text { such that } F_{1}=v F_{2} v^{T} \text {. }
$$

Then,

$$
A_{o}\left(F_{1}\right) \cong A_{o}\left(F_{2}\right) \text { if and only if } F_{1} \sim F_{2}
$$

Therefore, we will describe a fundamental domain for $\sim$ as is done in [6].

Proposition 5.3 A fundamental domain of $\sim$ is given by the following classes of matrices:

$$
\begin{aligned}
& \text { - }\left(\begin{array}{ccc}
0 & D\left(\lambda_{1}, \ldots, \lambda_{k}\right) & 0 \\
D\left(\lambda_{1}, \ldots, \lambda_{k}\right)^{-1} & 0 & 0 \\
0 & 0 & 1_{n-2 k}
\end{array}\right) \text { with } k, n \in \mathbb{N}, 2 k \leq n \text {, } \\
& 0<\lambda_{1} \leq \cdots \leq \lambda_{k}<1 \\
& \text { - }\left(\begin{array}{cc}
0 & D\left(\lambda_{1}, \ldots, \lambda_{n / 2}\right) \\
-D\left(\lambda_{1}, \ldots, \lambda_{n / 2}\right)^{-1} & 0
\end{array}\right) \text { with } 0<\lambda_{1} \leq \cdots \leq \lambda_{n / 2} \leq 1 \text {, } \\
& n \in \mathbb{N} \text { even. }
\end{aligned}
$$

Remark 5.4 Note that for $F \in \mathrm{GL}(2, \mathbb{C})$, up to equivalence, there only exists matrices of the form

$$
F_{q}=\left(\begin{array}{cc}
0 & |q|^{1 / 2} \\
-\operatorname{sgn}(q)|q|^{-1 / 2} & 0
\end{array}\right)
$$

for $q \in[-1,1] \backslash\{0\}$.

Definition 5.5 ([24,25]) Let $q \in[-1,1], q \neq 0$. Let $A$ be the universal unital $C^{*}$-algebra generated by two elements $\alpha, \gamma$ satisfying relations such that $U=$ $\left(\begin{array}{cc}\alpha & -q \gamma^{*} \\ \gamma & \alpha^{*}\end{array}\right) \in M_{2}(A)$ is a unitary matrix. With coproduct $\Delta\left(U_{i j}\right)=\sum_{k} U_{i k} \otimes U_{k j}$, $S U_{q}(2)=(A, \Delta)$ is a compact quantum group.

Proposition 5.6 With $F_{q}$ defined in Remark 5.4, we have $A_{o}\left(F_{q}\right) \cong S U_{q}(2)$.

Note that this last statement indeed implies that the only orthogonal quantum groups defined through matrices of dimension 2 are the quantized versions of $S U$ (2).

We state some results obtained by de Bichon et al. in [6] (Corollary 5.4 and Theorem $5.5)$.

Theorem 5.7 Let $F_{1} \in G L\left(n_{1}, \mathbb{C}\right)$ with $F_{1} \bar{F}_{1}=c_{1} 1, c_{1} \in \mathbb{R}$. Then, 
- a compact quantum group $\mathbb{G}$ is monoidally equivalent to $A_{o}\left(F_{1}\right)$ if and only if there exist a $F_{2} \in G L\left(n_{2}, \mathbb{C}\right)$ with $F_{2} \bar{F}_{2}=c_{2} 1, c_{2} \in \mathbb{R}$ and $\frac{c_{1}}{\operatorname{Tr}\left(F_{1}^{*} F_{1}\right)}=\frac{c_{2}}{\operatorname{Tr}\left(F_{2}^{*} F_{2}\right)}$ such that $\mathbb{G} \cong A_{o}\left(F_{2}\right)$.

- In this case, denote by $\mathcal{O}\left(A_{o}\left(F_{1}, F_{2}\right)\right)$ the *-algebra generated by the coefficients of $Y \in M_{n_{1}, n_{2}}(\mathbb{C}) \otimes \mathcal{O}\left(A_{o}\left(F_{1}, F_{2}\right)\right)$ with relations

$$
Y \text { is unitary and } Y=\left(F_{1} \otimes 1\right) \bar{Y}\left(F_{2}^{-1} \otimes 1\right) \text {, }
$$

then $\mathcal{O}\left(A_{o}\left(F_{1}, F_{2}\right)\right) \neq 0$ and it is the $\left(A_{o}\left(F_{1}\right)-A_{o}\left(F_{2}\right)\right)$-bi-Galois object with coactions $\beta_{1}$ of $\mathcal{O}\left(A_{o}\left(F_{1}\right)\right)$ and $\beta_{2}$ of $\mathcal{O}\left(A_{o}\left(F_{2}\right)\right)$ such that

$$
\left(\mathrm{id} \otimes \beta_{1}\right)(Y)=\left(U_{1}\right)_{12} Y_{13} \quad \text { and } \quad\left(\mathrm{id} \otimes \beta_{2}\right)(Y)=Y_{12}\left(U_{2}\right)_{13},
$$

where the $U_{i}$ are the unitary representations of $A_{o}\left(F_{i}\right)$, whose matrix coefficients generate the quantum groups.

- The monoidal equivalence preserves the dimensions if and only if $n_{2}=n_{1}$. In this case, we denote the unitary 2-cocycle by $\Omega\left(F_{2}\right)$. The $\Omega\left(F_{2}\right)$ describe up to equivalence all the unitary 2-cocycles on the dual of $A_{o}\left(F_{1}\right)$.

Remark 5.8 In [2], Banica shows that the irreducible representations of $A_{o}(F)$ can be labeled by $\mathbb{N}$ (say $r_{k}, k \in \mathbb{N}$ ). Moreover, for $\operatorname{dim}(F)=n$, he states that $\operatorname{dim}\left(r_{k}\right)=$ $\left(x^{k+1}-y^{k+1}\right) /(x-y)$, where $x$ and $y$ are solutions of $X^{2}-n X+1=0$ for $n \geq 3$ and $\operatorname{dim}\left(r_{k}\right)=k+1$ for $n=2$. Hence, it is easy to show by induction that if $\varphi$ is a monoidal equivalence between $S U_{q}(2)$ and $A_{o}(F)$ with $\operatorname{dim}(F) \geq 3$, then $\operatorname{dim}\left(\varphi\left(r_{k}\right)\right)>\operatorname{dim}\left(r_{k}\right)=k+1$ for every irreducible representation $r_{k}$ with $k \geq 1$.

Moreover, looking at the concrete orthogonal quantum group $S U_{q}(2)$, it is possible to classify all compact quantum groups which are monoidally equivalent to $S U_{q}(2)$ : indeed, applying the result of the last paragraph to the specific situation of $F=F_{q}$, we know exactly what the quantum groups are which are monoidal equivalent to $S U_{q}(2)$.

Proposition 5.9 ([6]) Let $0<q \leq 1$. For every even natural number $n$ with $2 \leq n \leq$ $q+1 / q$, there exists a monoidal equivalence on $S U_{q}(2)$ such that the multiplicity of the fundamental representation is $n$. Concretely, $S U_{q}(2) \sim_{\text {mon }} A_{o}(F)$ with $F=$ $\left(\begin{array}{cc}0 & D\left(\lambda_{1}, \ldots, \lambda_{n / 2}\right) \\ -D\left(\lambda_{1}, \ldots, \lambda_{n / 2}\right)^{-1} & 0\end{array}\right)$, where $0<\lambda_{1} \leq \cdots \leq \lambda_{n / 2} \leq 1$ and $\sum_{i=1}^{n / 2} \frac{1}{\lambda_{i}^{2}}+\lambda_{i}^{2}=q+1 / q$.

Let $0>q \geq-1$. Then for every natural number $n$ with $2 \leq n \leq|q+1 / q|$, there exists a monoidal equivalence on $S U_{q}(2)$ such that the multiplicity of the fundamental representation is $n$. Concretely, $S U_{q}(2) \sim_{\text {mon }} A_{o}(F)$ with $F=$ $\left(\begin{array}{ccc}0 & D\left(\lambda_{1}, \ldots, \lambda_{k}\right) & 0 \\ D\left(\lambda_{1}, \ldots, \lambda_{k}\right)^{-1} & 0 & 0 \\ 0 & 0 & 1_{n-2 k}\end{array}\right)$, where $k \in \mathbb{N}, 2 k \leq n, 0<\lambda_{1} \leq \cdots \leq$ $\lambda_{k}<1$ and $\sum_{i=1}^{k} \frac{1}{\lambda_{i}^{2}}+\lambda_{i}^{2}+n-2 k=|q+1 / q|$. 


\subsection{Monoidal deformation of the Podleś sphere}

In Sect. 4, we proved that our monoidal deformation of spectral triples is a generalization of the cocycle deformation, developed in [14]. In this subsection, we will give a concrete example to prove that our construction is a genuine generalization: we will construct a monoidal deformation of the Podleś sphere (with spectral triple of Dabrowski et al. [10]) which is not a 2-cocycle deformation. First, we recapitulate the definition of the Podleś sphere $S_{q, c}^{2}$ and the spectral triple on it. Then we will use the results of Sect. 5.1 to apply the construction of Sect. 3 .

\subsubsection{The Podleś sphere, its spectral triple and its quantum isometry group}

The Podleś sphere was initially constructed by Podleś in [18] as follows. Let $q \in$ $(-1,1) \backslash\{0\}$ and $t \in(0,1]$; hence $c=t^{-1}-t \geq 0$. We define $\mathcal{O}\left(S_{q, c}^{2}\right)$ to be the *-algebra generated by elements $A, B$ which satisfy the relations

$$
\begin{aligned}
A^{*}=A, & A B=q^{-2} B A, \\
B^{*} B=A-A^{2}+c 1, & B B^{*}=q^{2} A-q^{4} A^{2}+c 1 .
\end{aligned}
$$

One can see that for $q=1$, we have $A^{*}=A, A B=B A, B^{*} B=B B^{*}=A-A^{2}+c 1$ and this is the classical sphere: taking $A=z+1 / 2, B=x+i y, r^{2}=c+1 / 4$, we indeed have

$$
x^{2}+y^{2}+z^{2}=B^{*} B+A^{2}-A+1 / 4=c+1 / 4=r^{2} .
$$

The associated quantum space is called the Podleś sphere $S_{q, c}^{2}$.

Note first that for $q \in(0,1)$, setting

$$
x_{0}=t\left(1-\left(1+q^{2}\right) A\right), x_{-1}=\frac{t\left(1+q^{2}\right)^{\frac{1}{2}}}{q} B, x_{1}=-t\left(1+q^{2}\right)^{\frac{1}{2}} B^{*},
$$

we see that the definition in [10] with $\left\{x_{0}, x_{-1}, x_{1}\right\}$ is equivalent to the original definition of Podleś given above. Moreover, defining

$$
\begin{aligned}
& \tilde{A}=\frac{1+t^{-1} q \gamma^{*} \alpha-t^{-1} \rho\left(1-\left(1+q^{2}\right) \gamma^{*} \gamma\right)+t^{-1} \gamma \alpha^{*}}{1+q^{2}} \\
& \tilde{B}=\frac{q \alpha^{2}+\rho\left(1+q^{2}\right) \alpha \gamma-q^{2} \gamma^{2}}{t\left(1+q^{2}\right)}
\end{aligned}
$$

(where $\rho^{2}=\frac{q^{2} t^{2}}{\left(q^{2}+1\right)^{2}(1-t)}$ ) if $t \neq 1$ and

$$
\tilde{A}=\gamma^{*} \gamma, \quad \tilde{B}=q \alpha \gamma,
$$

if $t=1$, one can prove that the unital ${ }^{*}$-subalgebra of $C\left(S U_{q}(2)\right)$ generated by $\tilde{A}$ and $\tilde{B}$ is isomorphic to $\mathcal{O}\left(S_{q, c}^{2}\right)$ where $c=t^{-1}-t$, sending $A$ to $\tilde{A}$ and $B$ to $\tilde{B}$. 
Doing as above, we have three equivalent descriptions of the Podleś sphere.

The spectral triple on $S_{q, c}^{2}$ we use, is the spectral triple developed by Dabrowski et al. in [10]. The spectral triple uses the representation theory of $S U_{q}(2)$ described by Banica in [2]. To be compatible with [10], we use their notation. For each $n$ in $\{0,1 / 2,1, \ldots\}$, there exists a unique irreducible representation $D^{n}$ of $S U_{q}(2)\left(r_{2 n}\right.$ in Banica's notation) with dimension $2 n+1$. For example, we have

$$
D^{1 / 2}=\left(\begin{array}{cc}
\alpha & -q \gamma^{*} \\
\gamma & \alpha^{*}
\end{array}\right)
$$

and

$$
D^{1}=\left(\begin{array}{ccc}
\alpha^{* 2} & -\left(q^{2}+1\right) \alpha^{*} \gamma & -q \gamma^{2} \\
\gamma^{*} \alpha^{*} & 1-\left(q^{2}+1\right) \gamma^{*} \gamma & \alpha \gamma \\
-q \gamma^{* 2} & -\left(q^{2}+1\right) \gamma^{*} \alpha & \alpha^{2}
\end{array}\right)
$$

Denoting $d_{k, l}^{n}$ to be the $k, l$-matrix coefficient of $D^{n}$, one can prove that

$$
\left\{d_{k, l}^{n} \mid n=0, \frac{1}{2}, 1, \ldots ; k, l=-n,-n+1, \ldots, n-1, n\right\}
$$

form an orthogonal basis of $\mathcal{K}=L^{2}\left(S U_{q}(2), h\right)$, the GNS-space corresponding to the Haar state $h$ of $S U_{q}(2)$. Moreover, we will denote $e_{k, l}^{n}$ the multiples of $d_{k, l}^{n}$ such that $\left\{e_{k, l}^{n}\right\}$ form an orthonormal basis of $\mathcal{K}$.

Furthermore, consider the following closed subspace of $\mathcal{K}$ :

$$
\mathcal{H}:=\left[e_{ \pm \frac{1}{2}, l}^{n} \mid n=\frac{1}{2}, \frac{3}{2}, \ldots ; l=-n,-n+1, \ldots, n-1, n\right] .
$$

Then, one can prove that $\tilde{A}$ and $\tilde{B}$, as defined above, leave $\mathcal{H}$ invariant and we have a faithful *-morphism $\pi: \mathcal{O}\left(S_{q, c}^{2}\right) \rightarrow B(\mathcal{H}): A \mapsto \tilde{A}_{\left.\right|_{\mathcal{H}}}, B \mapsto \tilde{B}_{\left.\right|_{\mathcal{H}}}$, which makes it possible to identify $\mathcal{O}\left(S_{q, c}^{2}\right)$ with its image.

Finally, we can define an appropriate Dirac operator by setting

$$
D\left(e_{ \pm \frac{1}{2}, l}^{n}\right)=\left(c_{1} n+c_{2}\right) e_{\mp \frac{1}{2}, l}^{n},
$$

where $c_{1}, c_{2} \in \mathbb{R}, c_{1} \neq 0$ are arbitrary constants.

In [10], the authors prove that $\left(\mathcal{O}\left(S_{q, c}^{2}\right), \mathcal{H}, D\right)$ constitutes a well-defined spectral triple. As

$$
\Delta_{S U_{q}(2)}\left(e_{ \pm \frac{1}{2}, l}^{n}\right)=\sum_{k=-n,-n+1, \ldots, n} e_{ \pm \frac{1}{2}, k}^{n} \otimes e_{k, l}^{n}
$$

it is easy to see that $\Delta_{S U_{q}(2)}$ induces a unitary representation $U$ of $S U_{q}(2)$ on $\mathcal{H}$. By [10], the spectral triple is equivariant with respect to this representation 
and, hence, $S U_{q}(2)$ acts algebraically and by orientation-preserving isometries on $\left(\mathcal{O}\left(S_{q, c}^{2}\right), \mathcal{H}, D\right)$. We will use this representation and the monoidal equivalences of Sect. 5.1 to deform this spectral triple.

\subsubsection{Monoidal deformation of the Podleś sphere}

To conclude this section, we construct a non-dimension-preserving example. Now, we know that there is a well-defined spectral triple $\left(\mathcal{O}\left(S_{q, c}^{2}\right), \mathcal{H}, D\right)$ on which $S U_{q}(2)$ acts algebraically and by orientation-preserving isometries. Furthermore, we know from Proposition 5.9 what the monoidal equivalences of $S U_{q}(2)$ are and we know that those monoidal equivalences are non-dimension preserving by Remark 5.8. Putting all this together, we can apply the construction described in Sect. 3 to get the following theorem.

Theorem 5.10 Let $q \in[-1,1] \backslash\{0\}$ and $n$ be a natural number with $3 \leq n \leq \mid q+$ $1 / q \mid$.

If $q>0$ and $n$ is even, let $\lambda_{1}, \ldots, \lambda_{n / 2}$ be strict positive real numbers not bigger than 1 such that $\lambda_{1}^{2}+\cdots+\lambda_{n / 2}^{2}+1 / \lambda_{1}^{2}+\cdots+1 / \lambda_{n / 2}^{2}=q+1 / q$ and define $F$ to be the $n$ by $n$ matrix:

$$
F=\left(\begin{array}{ll}
0 & D\left(\lambda_{1}, \ldots, \lambda_{n / 2}\right) \\
-D\left(\lambda_{1}, \ldots, \lambda_{n / 2}\right)^{-1} & 0
\end{array}\right)
$$

If $0>q$, let $k$ be a natural number $k \leq n / 2$ and $\lambda_{1}, \ldots, \lambda_{k}$ be strict positive real numbers such that $0<\lambda_{1} \leq \cdots \leq \lambda_{k}<1$ and $\sum_{i=1}^{k} \frac{1}{\lambda_{i}^{2}}+\lambda_{i}^{2}+n-2 k=|q+1 / q|$ and define $F$ to be the $n$ by $n$ matrix

$$
F=\left(\begin{array}{lll}
0 & D\left(\lambda_{1}, \ldots, \lambda_{k}\right) & 0 \\
D\left(\lambda_{1}, \ldots, \lambda_{k}\right)^{-1} & 0 & 0 \\
0 & 0 & 1_{n-2 k}
\end{array}\right)
$$

With $F$ defined as above, there exists a non-dimension-preserving monoidal equivalence $\varphi$ from $S U_{q}(2)$ to $A_{o}(F)$ (introduced in Definition 5.1). Denoting by $\mathcal{O}\left(A_{o}\left(F_{q}, F\right)\right)$ the algebra constructed in 5.7, $\mathcal{O}\left(A_{o}\left(F_{q}, F\right)\right)$ is the associated biGalois object and the following triplet is a spectral triple:

$$
\left(\mathcal{O}\left(S_{q, c}^{2}\right) \underset{\mathcal{O}\left(S U_{q}(2)\right)}{\dot{O}} \mathcal{O}\left(A_{o}\left(F_{q}, F\right)\right), \quad \mathcal{H} \underset{C\left(S U_{q}(2)\right)}{\otimes} L^{2}\left(\mathcal{O}\left(A_{o}\left(F_{q}, F\right)\right)\right), \quad \tilde{D}\right)
$$

Moreover, $A_{o}(F)$ acts algebraically and by orientation-preserving isometries on the new spectral triple. As $\varphi$ is non-dimension preserving, it is not a 2-cocycle deformation à la Goswami and Joardar [14]. 


\section{Deformation of the quantum isometry group}

The goal of this last section is to prove that the deformation (in the sense of Theorem 3.12) of the quantum isometry group of a spectral triple (defined by Bhowmick and Goswami) is the quantum isometry group of the deformed spectral triple. We start by recalling some concepts and results of [4].

Definition 6.1 (Definition 2.7 in [4]) An $R$-twisted spectral triple (of compact type) is given by a triple $(\mathcal{A}, \mathcal{H}, D)$ and an operator $R$ on $\mathcal{H}$ where

1. $(\mathcal{A}, \mathcal{H}, D)$ is a compact spectral triple,

2. $R$ is a positive (possibly unbounded) invertible operator such that $R$ commutes with $D$.

Remark 6.2 We note that in Definition 2.7 in [4], there is a third condition in the definition of $R$-twisted spectral triple. However in remark 2.11 of [4], the authors state that this third condition is not necessary. Therefore, we gave the definition above.

Such an operator $R$ is linked with the preservation of a non-commutative analogue of a volume form.

Definition 6.3 ([4]) Let $R$ be a positive invertible operator and $(\mathcal{A}, \mathcal{H}, D)$ an $R$ twisted spectral triple. Then a compact quantum group $\mathbb{G}$ acting on $(\mathcal{A}, \mathcal{H}, D)$ by orientation-preserving isometries is said to preserve the $R$-twisted volume if one has

$$
\left(\tau_{R} \otimes \mathrm{id}\right)\left(\alpha_{U}(x)\right)=\tau_{R}(x) 1_{C(\mathbb{G})}
$$

for all $x \in \mathcal{E}_{D}$, where $\tau_{R}(x)=\operatorname{Tr}(R x)$ and $\mathcal{E}_{D}$ is the *-subalgebra of $B(\mathcal{H})$ generated by the rank-one operators of the form $\eta \xi^{*}, \eta, \xi$ eigenvectors of $D$.

In what follows, we will denote by $\mathcal{Q}_{R}(\mathcal{A}, \mathcal{H}, D)$ (or just $\mathcal{Q}_{R}$ ) the category of all compact quantum groups acting by $R$-twisted volume- and orientation-preserving isometries with the morphisms of quantum groups which are compatible with the representations on $\mathcal{H}$.

Moreover, one can prove (as is done in [12]) that for every compact quantum group acting by orientation-preserving isometries, there exists an operator $R$ such that the quantum group is an element of $\mathcal{Q}_{R}$.

Now, Goswami and Bhowmick proved in [4] that there exists a universal object in $\mathcal{Q}_{R}(\mathcal{A}, \mathcal{H}, D)$.

Theorem 6.4 (Theorem 2.14 in [4]) For any $R$-twisted spectral triple $(\mathcal{A}, \mathcal{H}, D)$, there exists a universal (initial) object $\left(\operatorname{QISO}_{R}^{0}(\mathcal{A}, \mathcal{H}, D), U_{0}\right)$ in the category $\mathcal{Q}_{R}$. The representation is faithful.

For notational convenience, we will write $\mathrm{QISO}_{\mathrm{R}}^{0}$ if there is no confusion possible about the spectral triple. However, in general $\alpha_{U_{0}}$ may not be faithful even if $U_{0}$ is so. Therefore, one has the following definition.

Definition 6.5 (Definition 2.16 in [4]) Let $\mathcal{C}=C^{*}\left(\left\{(f \otimes \mathrm{id}) \alpha_{U_{0}}(a) \mid a \in \mathcal{A}, f \in\right.\right.$ $\left.\mathcal{A}^{*}\right\}$ ) be the $C^{*}$-subalgebra of $C\left(\mathrm{QISO} \mathrm{R}_{\mathrm{R}}^{0}\right.$ ) generated by elements of the form $(f \otimes$ id) $\alpha_{U_{0}}(a), a \in \mathcal{A}$. 
Then, $\mathcal{C}$ is a Woronowicz $C^{*}$-subalgebra of $\mathrm{QISO}_{\mathrm{R}}^{0}$ and the compact quantum group

$$
\mathrm{QISO}_{R}(\mathcal{A}, \mathcal{H}, \mathcal{D})=\left(\mathcal{C}, \Delta_{\mathrm{QISO}_{\mathrm{R} \mid \mathcal{C}}^{0}}\right)
$$

is called the quantum group of $R$-twisted volume- and orientation-preserving isometries or simply quantum isometry group.

In Sect. 6.3, we will prove that if $(\mathcal{A}, \mathcal{H}, D)$ is an $R$-twisted spectral triple and

$$
\varphi: \operatorname{QISO}_{R}(\mathcal{A}, \mathcal{H}, \mathcal{D}) \rightarrow \mathbb{G}_{2}
$$

is a monoidal equivalence, then there exists an operator $\tilde{R}$ such that $(\tilde{\mathcal{A}}, \tilde{\mathcal{H}}, \tilde{D})$ is an $\tilde{R}$-spectral triple and $\mathbb{G}_{2}=\operatorname{QISO}_{\tilde{R}}(\tilde{\mathcal{A}}, \tilde{\mathcal{H}}, \tilde{D})$. But before we do that, we describe, given a monoidal equivalence $\varphi: \mathbb{G}_{1} \rightarrow \mathbb{G}_{2}$, how to construct a monoidal equivalence between certain Woronowicz- $C^{*}$-subalgebras (Sect. 6.1) resp. quantum supergroups (Sect. 6.2) of $\mathbb{G}_{1}$ and $\mathbb{G}_{2}$.

\subsection{Inducing monoidal equivalences on Woronowicz- $C^{*}$-subalgebras}

Definition 6.6 ([1]) Let $\mathbb{G}=(C(\mathbb{G}), \Delta)$ be a compact quantum group and $A$ a $C^{*}$ subalgebra of $C(\mathbb{G})$ such that $\Delta(A) \subset A \otimes A$ and $\left[\Delta_{\left.\right|_{A}}(A)(A \otimes 1)\right]=A \otimes A=$ $\left[\Delta_{\left.\right|_{A}}(A)(1 \otimes A)\right]$. Then, $A$ is called a Woronowicz $C^{*}$-subalgebra. We will write $\mathbb{A}=\left(A, \Delta_{\left.\right|_{A}}\right)$ for the quantum group.

It is good to remark that the notion of compact quantum quotient group introduced in [22] is a special case of a Woronowicz $C^{*}$-subalgebra. However, it is still unknown whether all Woronowicz $C^{*}$-subalgebras are compact quantum quotient groups.

In this section, let $\mathbb{G}=(C(\mathbb{G}), \Delta)$ be a $C Q G$ and $A$ a Woronowicz $C^{*}$-subalgebra of $\mathbb{G}$. To define a unitary fiber functor on $\mathbb{A}$, it is good to examine its representations. It is easy to see that every representation $U$ of $\mathbb{A}$ on a Hilbert space $\mathcal{H}$ is a representation of $\mathbb{G}$ and that every representation $V$ of $\mathbb{G}$ is a representation of $A$ if and only if $V \in \mathcal{M}(\mathcal{K}(\mathcal{H}) \otimes A)$. To distinguish, we will write $U_{\mathbb{G}}$ for a representation $U$ of $\mathbb{A}$ seen as representation of $\mathbb{G}$. Moreover, we have the following proposition:

Proposition 6.7 Let $U$ be a unitary representation of $\mathbb{A}$. Then, $U$ is irreducible if and only if $U_{\mathbb{G}}$ is irreducible.

Proof We know that $U$ resp. $U_{\mathbb{G}}$ is irreducible if and only if $\operatorname{Mor}(U, U)=\{T \in$ $B(\mathcal{H}) \mid(T \otimes \mathrm{id}) U=U(T \otimes \mathrm{id})\}$ resp. $\operatorname{Mor}\left(U_{\mathbb{G}}, U_{\mathbb{G}}\right)$ equals $\mathbb{C} 1_{B(\mathcal{H})}$. As it is directly clear that $\operatorname{Mor}(U, U)=\operatorname{Mor}\left(U_{\mathbb{G}}, U_{\mathbb{G}}\right)$, the proposition is proved.

Analogously as before, we will write $x_{\mathbb{G}}$ if we look at the equivalence class $x \in$ $\operatorname{Irred}(\mathbb{A})$ seen as equivalence class in $\operatorname{Irred}(\mathbb{G})$. Using this proposition, the unitary fiber functor is easily made: let $\mathbb{G}_{1}$ be a compact quantum group and $\varphi: \mathbb{G}_{1} \rightarrow \mathbb{G}_{2}$ a monoidal equivalence between them. Suppose, moreover, that $A_{1}$ is a Woronowicz subalgebra of $\mathbb{G}_{1}$. Then, we can construct a unitary fiber functor on $\mathbb{A}_{1}=\left(A_{1}, \Delta_{\left.\right|_{A_{1}}}\right)$ by restricting $\varphi$ to the representations of $\mathbb{A}$ and prove it is a monoidal equivalence 
between $\mathbb{A}_{1}$ and a compact quantum group $\mathbb{A}_{2}$ such that $C\left(\mathbb{A}_{2}\right)$ is a Woronowciz $C^{*}$-algebra of $\mathbb{G}_{2}$.

Proposition 6.8 Let $\mathbb{G}_{1}$ be a compact quantum group, $A_{1}$ a Woronowicz $C^{*}$ subalgebra of $\mathbb{G}_{1}$ and $\psi$ a unitary fiber functor on $\mathbb{G}_{1}$. Then there exists a unitary fiber functor $\psi^{\prime}$ on $\mathbb{A}_{1}=\left(A_{1}, \Delta_{\left.1\right|_{A_{1}}}\right)$ such that $\psi^{\prime}(x)=\psi\left(x_{\mathbb{G}_{1}}\right)$ for all $x \in \operatorname{Irred}\left(\mathbb{A}_{1}\right)$.

Proof Let $x \in \operatorname{Irred}\left(\mathbb{A}_{1}\right)$. Define $\mathcal{H}_{\psi^{\prime}(x)}$ to be $\mathcal{H}_{\psi\left(x_{\mathbb{G}}\right)}$ and $\psi^{\prime}(S)=\psi(S)$ for every $S \in \operatorname{Mor}\left(y_{1} \otimes \cdots \otimes y_{k}, x_{1} \otimes \cdots \otimes x_{r}\right), y_{1}, \ldots, y_{k}, x_{1}, \ldots, x_{r} \in \operatorname{Irred}\left(\mathbb{A}_{1}\right)$. As $\psi$ is a unitary fiber functor, $\psi^{\prime}$ will satisfy all the necessary conditions to be a unitary fiber functor as well.

Denoting by $\varphi: \mathbb{G}_{1} \rightarrow \mathbb{G}_{2}$ the monoidal equivalence associated with $\psi$; we can see that $C\left(\mathbb{G}_{2}\right)$ as the $C^{*}$-algebra generated (as vector space) by the coefficients of the $U^{\varphi(x)}, x \in \operatorname{Irred}\left(\mathbb{G}_{1}\right)$. Now, we can define $A_{2}$ as the $C^{*}$-algebra generated (as vector space) by the coefficients of the $U^{\varphi\left(x_{\mathbb{G}_{1}}\right)}, x \in \operatorname{Irred}\left(\mathbb{A}_{1}\right)$. Equivalently,

$$
A_{2}=\left[(\omega \otimes \mathrm{id}) U^{\varphi\left(x_{\mathbb{G}_{1}}\right)} \mid x \in \operatorname{Irred}\left(\mathbb{A}_{1}\right)\right]
$$

and we also write

$$
\mathcal{A}_{2}=\left\langle(\omega \otimes \mathrm{id}) U^{\varphi\left(x_{\mathbb{G}_{1}}\right)} \mid x \in \operatorname{Irred}\left(\mathbb{A}_{1}\right)\right\rangle .
$$

Note that they are indeed algebras: if $x, y \in \operatorname{Irred}\left(\mathbb{A}_{1}\right)$, then the product of a matrix coefficient of $U^{\varphi(x)}$ and one of $U^{\varphi(y)}$ is a matrix coefficient of the tensor product of them. By construction, this tensor product is a direct sum of representations $\varphi\left(z_{i}\right)$ where all $z_{i}$ are in $\operatorname{Irred}\left(\mathbb{A}_{1}\right)$.

Now, it is clear that $\psi^{\prime}$ induces a monoidal equivalence $\varphi^{\prime}$ between $\mathbb{A}_{1}$ and a compact quantum group with algebra $A_{2}$.

Theorem 6.9 With the map $\Delta_{2}^{\prime}=\Delta_{\left.2\right|_{A_{2}}}, \mathbb{A}_{2}=\left(A_{2}, \Delta_{2}^{\prime}\right)$ is a compact quantum group. Moreover the monoidal equivalence $\varphi^{\prime}$, induced by $\psi$, is an equivalence between $\mathbb{A}_{1}$ and $\mathbb{A}_{2}$.

Proof Written differently, $A_{2}$ is the closed linear span of the elements $u_{i j}^{\varphi\left(x_{\mathbb{G}_{1}}\right)}, x \in$ $\operatorname{Irred}\left(\mathbb{A}_{1}\right)$. For $\Delta_{2}^{\prime}$ defined as above, we get:

$$
\Delta_{2}^{\prime}\left(u_{i j}^{\varphi\left(x_{\mathbb{G}_{1}}\right)}\right)=\sum_{k} u_{i k}^{\varphi\left(x_{\mathbb{G}_{1}}\right)} \otimes u_{k j}^{\varphi\left(x_{\mathbb{G}_{1}}\right)},
$$

and as $x \in \operatorname{Irred}\left(\mathbb{A}_{1}\right)$, we see that $\Delta_{2}^{\prime}\left(A_{2}\right) \subset A_{2} \otimes A_{2}$. Now, denote by $\varepsilon^{\prime}$ and $S^{\prime}$ the restrictions of the counit $\varepsilon$ and antipode $S$ of $\mathbb{G}_{2}$ defined on $\mathcal{O}\left(\mathbb{G}_{2}\right)$ to $\mathcal{A}_{2}$. Then, $\mathcal{A}_{2}=\left\langle(\omega \otimes \mathrm{id}) U^{\varphi\left(x_{\mathbb{G}_{1}}\right)} \mid x \in \operatorname{Irred}\left(\mathbb{A}_{1}\right)\right\rangle=\mathcal{O}\left(\mathbb{A}_{2}\right)$ is a Hopf *algebra which is dense in $A_{2}$. This proves that $\mathbb{A}_{2}=\left(A_{2}, \Delta_{2}^{\prime}\right)$ is indeed a compact quantum group. By construction of $\varphi^{\prime}$, it is evident that it is a monoidal equivalence between $\mathbb{A}_{1}$ and $\mathbb{A}_{2}$. 
Before we go the next paragraph, we want to explore how the $\left(\mathbb{G}_{1}-\mathbb{G}_{2}\right)$-bi-Galois object behaves with respect to the $\left(\mathbb{A}_{1}-\mathbb{A}_{2}\right)$-bi-Galois object .

Theorem 6.10 Let $\mathbb{G}_{1}, \mathbb{G}_{2}, \mathbb{A}$ be compact quantum groups such that $C(\mathbb{A})$ is a Woronowicz $C^{*}$-subalgebra of $C\left(\mathbb{G}_{1}\right)$ and such that $\varphi: \mathbb{G}_{1} \rightarrow \mathbb{G}_{2}$ is a monoidal equivalence. Let $\mathcal{B}$ be the $\mathbb{G}_{1}-\mathbb{G}_{2}$-bi-Galois object with $\beta_{1}: \mathcal{B} \rightarrow \mathcal{O}\left(\mathbb{G}_{1}\right) \odot \mathcal{B}$. Let $\varphi^{\prime}$ be the monoidal equivalence between $\mathbb{A}_{1}$ and $\mathbb{A}_{2}$ as defined above and define $\mathcal{B}^{\prime}$ to be the $\mathbb{A}_{1}-\mathbb{A}_{2}$-bi-Galois object with $\gamma_{1}: \mathcal{B}^{\prime} \rightarrow \mathcal{O}\left(\mathbb{A}_{1}\right) \odot \mathcal{B}^{\prime}$. Then we have

$$
\mathcal{B}^{\prime}=\left\{b \in \mathcal{B} \mid \beta_{1}(b) \in \mathcal{O}\left(\mathbb{A}_{1}\right) \odot \mathcal{B}\right\}
$$

and $\gamma_{1}=\beta_{\left.1\right|_{\mathcal{B}^{\prime}}}$.

Proof From the original proof of Theorem 2.13 (which is theorem 3.9 in [6]), we know that $\mathcal{B}^{\prime}=\oplus_{x \in \operatorname{Irred}\left(\mathbb{A}_{1}\right)} B\left(\mathcal{H}_{\varphi(x)}, \mathcal{H}_{x}\right)^{*}$ and $\mathcal{B}=\oplus_{x \in \operatorname{Irred}\left(\mathbb{G}_{1}\right)} B\left(\mathcal{H}_{\varphi(x)}, \mathcal{H}_{x}\right)^{*}$. Hence, $\mathcal{B}^{\prime} \subset \mathcal{B}$. Also, $X^{x} \in B\left(\mathcal{H}_{\varphi(x)}, \mathcal{H}_{x}\right) \odot \mathcal{B}$ is defined such that $\left(\omega_{x} \otimes \mathrm{id}\right)\left(X^{x}\right)=$ $\left(\delta_{x, y} \omega_{x}\right)_{y \in \operatorname{Irred}\left(\mathbb{G}_{1}\right)}$ for all $\omega_{x} \in B\left(\mathcal{H}_{\varphi(x)}, \mathcal{H}_{x}\right)^{*}$. By definition, we see that for $x \in$ $\operatorname{Irred}\left(\mathbb{A}_{1}\right), X^{x}=X^{x_{\mathbb{G}_{1}}}$. As $\beta_{1}$ resp. $\gamma_{1}$ are defined by $\left(\mathrm{id} \otimes \beta_{1}\right)\left(X^{x}\right)=U_{12}^{x} X_{13}^{x}$ $\left(x \in \operatorname{Irred}\left(\mathbb{G}_{1}\right)\right)$ resp. $\left(\mathrm{id} \otimes \gamma_{1}\right)\left(X^{x}\right)=U_{12}^{x} X_{13}^{x}\left(x \in \operatorname{Irred}\left(\mathbb{A}_{1}\right)\right)$, it follows directly that $\gamma_{1}=\left(\beta_{1}\right)_{\left.\right|_{\mathcal{B}^{\prime}}}$. Moreover, if $x \in \operatorname{Irred}\left(\mathbb{A}_{1}\right), U_{12}^{x} X_{13}^{x} \in B\left(\mathcal{H}_{\varphi(x)}, \mathcal{H}_{x}\right) \odot \mathcal{O}\left(\mathbb{A}_{1}\right) \odot \mathcal{B}$ and hence for $b \in \mathcal{B}^{\prime}, \beta_{1}(b) \in \mathcal{O}\left(\mathbb{A}_{1}\right) \odot \mathcal{B}$. If $x \in \operatorname{Irred}\left(\mathbb{G}_{1}\right)$ but $x \notin \operatorname{Irred}\left(\mathbb{A}_{1}\right)$, $U_{12}^{x} X_{13}^{x} \notin B\left(\mathcal{H}_{\varphi(x)}, \mathcal{H}_{x}\right) \odot \mathcal{O}\left(\mathbb{A}_{1}\right) \odot \mathcal{B}$ and hence for $b \in \mathcal{B}$ but $b \notin \mathcal{B}^{\prime}, \beta_{1}(b) \notin$ $\mathcal{O}\left(\mathbb{A}_{1}\right) \odot \mathcal{B}$. This concludes the proof.

Remark 6.11 In the special case of compact quantum quotient groups, a compact quantum quotient group of $\mathbb{G}_{1}$ will be monoidally equivalent to a compact quantum group which has as algebra a Woronowicz $C^{*}$-subalgebra of $\mathbb{G}_{2}$. Whether that compact quantum group is a compact quantum quotient group as well is still unknown [22].

\subsection{Inducing monoidal equivalences on supergroups}

In this subsection we describe, given a monoidal equivalence $\varphi: \mathbb{G}_{1} \rightarrow \mathbb{G}_{2}$, how to construct a monoidal equivalence between certain quantum supergroups of $\mathbb{G}_{1}$ and $\mathbb{G}_{2}$.

So, let $\mathbb{G}_{1}$ and $\mathbb{G}_{2}$ be two compact quantum groups and let $\varphi: \mathbb{G}_{1} \rightarrow \mathbb{G}_{2}$ be a monoidal equivalence. Moreover, suppose $\mathbb{G}_{1}$ is a compact quantum subgroup of a compact quantum group $\mathbb{H}_{1}$. As we have done in Sect. 6.1 for Woronowicz $C^{*}$ subalgebras, we will describe a method to construct a unitary fiber functor on $\mathbb{H}_{1}$ from the monoidal equivalence $\varphi$.

Let $\pi: C_{u}\left(\mathbb{H}_{1}\right) \rightarrow C_{u}\left(\mathbb{G}_{1}\right)$ be the surjective morphism which is compatible with the quantum group structure. Now note that for a representation $U$ of $\mathbb{H}$ on a Hilbert space $\mathcal{H},\left(\operatorname{id}_{\mathcal{H}} \otimes \pi\right) U$ is a representation of $\mathbb{G}_{1}$. Therefore, for $x \in \operatorname{Irred}\left(\mathbb{H}_{1}\right)$, define $x_{\mathbb{G}_{1}}$ to be the equivalence class of (id $\left.\otimes \pi\right) U^{x}$ as representation of $\mathbb{G}_{1}$ and

- if (id $\otimes \pi) U^{x}$ is irreducible, let $\mathcal{H}_{x_{\mathbb{G}_{1}}}=\mathcal{H}_{x}$;

- If (id $\otimes \pi) U^{x}$ is reducible, say (id $\left.\otimes \pi\right) U^{x}=\oplus_{i=1}^{n} U^{y_{i}}, y_{i} \in \operatorname{Irred}\left(\mathbb{G}_{1}\right)$, then let $\mathcal{H}_{\mathbb{G}_{1}}=\oplus_{i=1}^{n} \mathcal{H}_{y_{i}}$. 
If $x^{1}, \ldots, x^{r}, y^{1}, \ldots, y^{s}$ are classes of irreducible representations of $\mathbb{H}_{1}$ with $U^{x_{\mathbb{G}_{1}}^{i}}=$ $\oplus_{j_{i}} U^{z_{j_{i}}^{i}}$ and $U^{y_{\mathbb{G}_{1}}^{i}}=\oplus_{k_{i}} U^{t_{k_{i}}^{i}}$, we denote for a morphism $S \in \operatorname{Mor}\left(x_{1} \otimes \cdots \otimes\right.$ $\left.x_{r}, y_{1} \otimes \ldots, y_{s}\right), S_{\mathbb{G}_{1}}=\bigoplus_{j_{1}, \ldots, j_{r}, k_{1}, \ldots k_{s}} S_{k_{1}, \ldots, k_{s}}^{j_{1}, \ldots, j_{r}}$ to be the morphism $S$, but seen as element of $\bigoplus_{j_{1}, \ldots, j_{r}, k_{1}, \ldots k_{s}} \operatorname{Mor}\left(z_{k_{1}}^{1} \otimes \cdots \otimes z_{k_{s}}^{s}, t_{j_{1}}^{1} \otimes \cdots \otimes t_{j_{r}}^{r}\right)$, i.e. $S_{k_{1}, \ldots, k_{s}}^{j_{1}, \ldots, j_{r}} \in$ $\operatorname{Mor}\left(z_{k_{1}}^{1} \otimes \cdots \otimes z_{k_{s}}^{s}, t_{j_{1}}^{1} \otimes \cdots \otimes t_{j_{r}}^{r}\right)$.

Then, we can define the following map:

Proposition 6.12 Let $\mathbb{G}_{1}, \mathbb{G}_{2}, \mathbb{H}_{1}$ and $\varphi$ be as above. For $x \in \operatorname{Irred}\left(\mathbb{H}_{1}\right)$ with $U^{x_{\mathbb{G}_{1}}}=$ $(\mathrm{id} \otimes \pi) U^{x}=\oplus_{i=1}^{n} U^{y_{i}}, y_{i} \in \operatorname{Irred}\left(\mathbb{G}_{1}\right)$, define $\mathcal{H}_{\psi^{\prime}(x)}=\oplus_{i=1}^{n} \mathcal{H}_{\varphi\left(y_{i}\right)}$ and for $S \in$ $\operatorname{Mor}\left(x_{1} \otimes \cdots \otimes x_{r}, y_{1} \otimes \ldots, y_{s}\right)$ with $S_{\mathbb{G}_{1}}=\bigoplus_{j_{1}, \ldots, j_{r}, k_{1}, \ldots k_{s}} S_{k_{1}, \ldots, k_{s}}^{j_{1}, \ldots, j_{r}}$, let $\psi^{\prime}(S)=$ $\bigoplus_{j_{1}, \ldots, j_{r}, k_{1}, \ldots k_{s}} \varphi\left(S_{k_{1}, \ldots k_{s}}^{j_{1}, \ldots, j_{r}}\right)$. Then the collection of maps

$$
\mathcal{H}_{x} \mapsto \mathcal{H}_{\psi^{\prime}(x)} \quad S \in \operatorname{Mor}\left(x_{1} \otimes \cdots \otimes x_{r}, y_{1} \otimes \ldots, y_{s}\right) \mapsto \psi^{\prime}(S)
$$

constitutes a unitary fiber functor $\psi^{\prime}$ on $\mathbb{H}_{1}$.

The proof follows directly by construction of $\mathcal{H}_{\psi^{\prime}}$ and $\psi^{\prime}(S)$. By Theorem 2.12, there exists a compact quantum group $\mathbb{H}_{2}$ and a monoidal equivalence $\varphi^{\prime}: \mathbb{H}_{1} \rightarrow \mathbb{H}_{2}$. In Theorem 6.13, we will describe the bi-Galois object associated with $\varphi$ and the compact quantum group $\mathbb{H}_{2}$ explicitly.

Theorem 6.13 Let $\mathbb{G}_{1}, \mathbb{G}_{2}, \mathbb{H}_{1}$ be compact quantum groups such that $\mathbb{G}_{1}$ is a compact quantum subgroup of $\mathbb{H}_{1}$ with surjective morphism $\pi: C_{u}\left(\mathbb{H}_{1}\right) \rightarrow C_{u}\left(\mathbb{G}_{1}\right)$. Let $\varphi: \mathbb{G}_{1} \rightarrow \mathbb{G}_{2}$ be a monoidal equivalence as above and let $\mathbb{H}_{2}$ and $\varphi^{\prime}: \mathbb{H}_{1} \rightarrow \mathbb{H}_{2}$ be the compact quantum group and monoidal equivalence induced by $\varphi$ by Propositions 6.12 and 2.12 . Denoting by $\mathcal{B}$ the $\left(\mathbb{G}_{1}-\mathbb{G}_{2}\right)$-bi-Galois object associated with $\varphi$, by $\tilde{\mathcal{B}}$ the $\left(\mathbb{G}_{2}-\mathbb{G}_{1}\right)$-bi-Galois object associated with $\varphi^{-1}$ and by $\mathcal{B}^{\prime}$ the $\left(\mathbb{H}_{1}-\mathbb{H}_{2}\right)$-bi-Galois object associated with $\varphi^{\prime}$, we have

$$
\mathcal{B}^{\prime} \cong \mathcal{O}\left(\mathbb{H}_{1}\right) \underset{\mathcal{O}\left(\mathbb{G}_{1}\right)}{\ominus} \mathcal{B}
$$

and

$$
\mathcal{O}\left(\mathbb{H}_{2}\right) \cong \tilde{\mathcal{B}} \underset{\mathcal{O}\left(\mathbb{G}_{1}\right)}{\ominus_{(1)}} \mathcal{O}\left(\mathbb{H}_{1}\right) \underset{\mathcal{O}\left(\mathbb{G}_{1}\right)}{\ominus_{1}} \mathcal{B}
$$

using the right resp. left coactions $(\mathrm{id} \otimes \pi) \Delta_{\mathbb{H}_{1}}: \mathcal{O}\left(\mathbb{H}_{1}\right) \rightarrow \mathcal{O}\left(\mathbb{H}_{1}\right) \odot \mathcal{O}\left(\mathbb{G}_{1}\right)$ resp. $(\pi \otimes \mathrm{id}) \Delta_{\mathbb{H}_{1}}: \mathcal{O}\left(\mathbb{H}_{1}\right) \rightarrow \mathcal{O}\left(\mathbb{G}_{1}\right) \odot \mathcal{O}\left(\mathbb{H}_{1}\right)$ of $\mathcal{O}\left(\mathbb{G}_{1}\right)$ on $\mathcal{O}\left(\mathbb{H}_{1}\right)$.

Proof Let $X^{x}, x \in \operatorname{Irred}\left(\mathbb{G}_{1}\right)$ be the unitaries from Theorem 2.13 associated with $\varphi$. Define for $x \in \operatorname{Irred}\left(\mathbb{H}_{1}\right), X^{x_{\mathbb{G}_{1}}}=\bigoplus_{i=1}^{n} X^{y_{i}}$ if $U^{x_{\mathbb{G}_{1}}}=(\mathrm{id} \otimes \pi) U^{x}=\bigoplus_{i=1}^{n} U^{y_{i}}$, $y_{i} \in \operatorname{Irred}\left(\mathbb{G}_{1}\right)$. Moreover, define for $x \in \operatorname{Irred}\left(\mathbb{H}_{1}\right)$,

$$
Y^{x}=U_{12}^{x} X_{13}^{x_{\mathbb{G}_{1}}} \in B\left(\mathcal{H}_{\varphi^{\prime}(x)}, \mathcal{H}_{x}\right) \odot \mathcal{O}\left(\mathbb{H}_{1}\right) \odot \mathcal{B} .
$$


We claim that the $Y^{x}$ with the functional $\omega^{\prime}=h_{\mathbb{H}_{1}} \otimes \omega\left(h_{\mathbb{H}_{1}}\right.$ is the Haar state of $\mathbb{H}_{1}$ ) satisfy the properties $1(\mathrm{a}), 1(\mathrm{~b})$ and 1 (c) of Theorem 2.13 applied to $\varphi^{\prime}$. Indeed, we have for $x, y, z \in \operatorname{Irred}\left(\mathbb{H}_{1}\right)$ and $S \in \operatorname{Mor}(y \otimes z, x)$,

$$
\begin{aligned}
Y_{13}^{y} Y_{23}^{z}\left(\varphi^{\prime}(S) \otimes \mathrm{id}\right) & =U_{13}^{y} X_{14}^{y_{\mathbb{G}_{1}}} U_{23}^{z} X_{24}^{z_{\mathbb{G}_{1}}}\left(\varphi^{\prime}(S) \otimes \mathrm{id}\right) \\
& =U_{13}^{y} U_{23}^{z} X_{14}^{y_{\mathbb{G}_{1}}} X_{24}^{z_{\mathbb{G}_{1}}}\left(\varphi^{\prime}(S) \otimes \mathrm{id}\right) \\
& =U_{13}^{y} U_{23}^{z}(S \otimes \mathrm{id}) X_{13}^{x_{\mathbb{G}_{1}}} \\
& =(S \otimes \mathrm{id}) U_{12}^{x} X_{13}^{x_{\mathbb{G}_{1}}} \\
& =(S \otimes \mathrm{id}) Y^{x}
\end{aligned}
$$

Moreover, $\left(\mathrm{id} \otimes \omega^{\prime}\right) Y^{x}=\left(\mathrm{id} \otimes h_{\mathbb{H}_{1}} \otimes \omega\right)\left(U_{12}^{x} X_{13}^{x_{\mathbb{G}_{1}}}\right)=0$ if $x \neq \varepsilon$.

Hence to prove (6.1), it suffices to prove that the matrix coefficients of the $Y^{x}$ constitute a linear basis of $\mathcal{O}\left(\mathbb{H}_{1}\right) \square \mathcal{B}$. Note first that the matrix coefficients of the $Y^{x}$ are elements of $\mathcal{O}\left(\mathbb{H}_{1}\right) \underset{\mathcal{O}\left(\mathbb{G}_{1}\right)}{\square} \mathcal{B}$. Indeed,

$$
\begin{aligned}
& \left(\mathrm{id} \otimes\left(\operatorname{id}_{\mathcal{O}\left(\mathbb{H}_{1}\right)} \otimes \pi\right) \Delta_{\mathbb{H}_{1}} \otimes \operatorname{id}_{\mathcal{B}}\right) U_{12}^{x} X_{13}^{x_{\mathbb{G}_{1}}} \\
& \quad=U_{12}^{x} U_{13}^{x_{\mathbb{G}_{1}}} X_{14}^{x_{\mathbb{G}_{1}}}=\left(\mathrm{id} \otimes \operatorname{id}_{\mathcal{O}\left(\mathbb{H}_{1}\right)} \otimes \beta_{1}\right) U_{12}^{x} X_{13}^{x_{\mathbb{G}_{1}}} .
\end{aligned}
$$

Moreover, as every irreducible representation of $\mathbb{G}_{1}$ is a subrepresentation of some $x_{\mathbb{G}_{1}}, x \in \operatorname{Irred}\left(\mathbb{H}_{1}\right)$, the matrix coefficients of the $X^{x_{\mathbb{G}_{1}}}$ resp. the $U^{x}$ form a basis of $\mathcal{B}$ resp. $\mathcal{O}\left(\mathbb{H}_{1}\right)$. Hence, the matrix coefficients of the $Y^{x}$ are linearly independent. Finally, we prove that they are also generating. Let $z$ be an arbitrary element of $\mathcal{O}\left(\mathbb{H}_{1}\right) \quad \square \mathcal{B}$. Then, $z$ is of the form $\sum \lambda_{s t}^{i j} u_{i j}^{x} \otimes b_{s t}^{y}$ where the $u_{i j}^{x}$ resp. $b_{s t}^{y}$ are the matrix coefficients of the $U^{x}$ resp. $X^{y}, x \in \operatorname{Irred}\left(\mathbb{H}_{1}\right), y \in \operatorname{Irred}\left(\mathbb{G}_{1}\right)$ and $\lambda_{s t}^{i j} \in \mathbb{C}$. As $z \in \mathcal{O}\left(\mathbb{H}_{1}\right) \square \quad \dot{B}$, $\sum \lambda_{s t}^{i j} u_{i k}^{x} \otimes \pi\left(u_{k j}^{x}\right) \otimes b_{s t}^{y}=\sum_{x_{\mathbb{G}}} \lambda_{s t}^{i j} u_{i j}^{x} \otimes u_{s r}^{y} \otimes b_{r t}^{y}$, hence $z$ is a linear combination of matrix coefficients of $U_{12}^{x} X_{13}^{x_{\mathbb{G}_{1}}}$. As the unitaries satisfying properties $1(\mathrm{a}), 1(\mathrm{~b})$ and 1(c) of Theorem 2.13 are unique, the $Y^{x}$ are those unitaries and $\mathcal{B}^{\prime} \cong \mathcal{O}\left(\mathbb{H}_{1}\right) \underset{\mathcal{O}\left(\mathbb{G}_{1}\right)}{\square} \mathcal{B}$. This concludes the proof of the first result (6.1).

For the second result 6.2 , let $Z^{y}, y \in \operatorname{Irred}\left(\mathbb{G}_{2}\right)$ be the unitaries from Theorem 2.13 associated with $\varphi^{-1}$. If $U^{x_{\mathbb{G}_{1}}}=(\mathrm{id} \otimes \pi) U^{x}=\oplus_{i} U^{y_{i}}$ for $x \in \operatorname{Irred}\left(\mathbb{H}_{1}\right), y_{i} \in$ $\operatorname{Irred}\left(\mathbb{G}_{1}\right)$, we will denote $U^{\varphi\left(x_{\mathbb{G}_{1}}\right)}=\oplus_{i} U^{\varphi\left(y_{i}\right)}$ and $Z^{\varphi\left(x_{\mathbb{G}_{1}}\right)}=\oplus_{i} Z^{\varphi\left(y_{i}\right)} \in$ $B\left(\mathcal{H}_{x}, \mathcal{H}_{\varphi^{\prime}(x)}\right) \odot \tilde{\mathcal{B}}$.

Therefore, we can define

$$
V^{\varphi^{\prime}(x)}=Z_{12}^{\varphi\left(x_{\mathbb{G}_{1}}\right)} U_{13}^{x} X_{14}^{x_{\mathbb{G}_{1}}}
$$

Then, one can prove analogously as above that for $x, y, z \in \operatorname{Irred}\left(\mathbb{H}_{1}\right)$ and $S \in$ $\operatorname{Mor}(y \otimes z, x)$, 


$$
\begin{aligned}
V_{13}^{\varphi^{\prime}(y)} V_{23}^{\varphi^{\prime}(z)}\left(\varphi^{\prime}(S) \otimes \mathrm{id}\right) & =Z_{13}^{\varphi\left(y_{\mathbb{G}_{1}}\right)} U_{14}^{y} X_{15}^{y_{\mathbb{G}_{1}}} Z_{23}^{\varphi\left(z_{\mathbb{G}_{1}}\right)} U_{24}^{z} X_{25}^{z_{\mathbb{G}_{1}}}\left(\varphi^{\prime}(S) \otimes \mathrm{id}\right) \\
& =Z_{13}^{\varphi\left(y_{\mathbb{G}_{1}}\right)} Z_{23}^{\varphi\left(z_{\mathbb{G}_{1}}\right)} U_{14}^{y} U_{24}^{z} X_{15}^{y_{\mathbb{G}_{1}}} X_{25}^{z_{\mathbb{G}_{1}}}\left(\varphi^{\prime}(S) \otimes \mathrm{id}\right) \\
& =Z_{13}^{\varphi\left(y_{\mathbb{G}_{1}}\right)} Z_{23}^{\varphi\left(z_{\mathbb{G}_{1}}\right)} U_{14}^{y} U_{24}^{z}(S \otimes \mathrm{id}) X_{14}^{x_{\mathbb{G}_{1}}} \\
& =Z_{13}^{\varphi\left(y_{\mathbb{G}_{1}}\right)} Z_{23}^{\varphi\left(z_{\mathbb{G}_{1}}\right)}(S \otimes \mathrm{id}) U_{13}^{x} X_{14}^{x_{\mathbb{G}_{1}}} \\
& =\left(\varphi^{\prime}(S) \otimes \mathrm{id}\right) Z_{12}^{\varphi\left(x_{\mathbb{G}_{1}}\right)} U_{13}^{x} X_{14}^{x_{\mathbb{G}_{1}}} \\
& =\left(\varphi^{\prime}(S) \otimes \mathrm{id}\right) V^{\varphi^{\prime}(x)} .
\end{aligned}
$$

The argument to prove that the matrix coefficients of $V^{\varphi^{\prime}(x)}$ form a linear basis of $C\left(\mathbb{H}_{2}\right)$ is the same as in the first part of the proof.

Moreover, the newly constructed compact quantum group $\mathbb{H}_{2}$ is a supergroup of $\mathbb{G}_{2}$.

Proposition 6.14 We have a surjective morphism of compact quantum groups $\pi^{\prime}$ : $C_{u}\left(\mathbb{H}_{2}\right) \rightarrow C_{u}\left(\mathbb{G}_{2}\right)$ such that

$$
\left(\mathrm{id} \otimes \pi^{\prime}\right) V^{\varphi^{\prime}(x)}=U^{\varphi\left(x_{\mathbb{G}_{1}}\right)}
$$

for every $x \in \operatorname{Irred}\left(\mathbb{H}_{1}\right)$, implying that $\mathbb{G}_{2}$ is a quantum subgroup of $\mathbb{H}_{2}$.

Proof The map $\pi^{\prime}$ is well defined by (6.4) as the matrix coefficients of the $V^{\varphi^{\prime}(x)}$ constitute a linear basis of $\mathcal{O}\left(\mathbb{H}_{2}\right)$. Moreover, it is a linear surjection and it follows directly that it is coalgebra map. It suffices to prove that $\pi^{\prime}$ is an algebra map. Therefore, denoting by $f: \mathcal{O}\left(\mathbb{G}_{2}\right) \rightarrow \widetilde{B} \underset{\mathcal{O}\left(\mathbb{G}_{1}\right)}{\square} \mathcal{B}$ the isomorphism of Proposition 2.18 (applied to $\left.\varphi^{-1}: \mathbb{G}_{2} \rightarrow \mathbb{G}_{1}\right)$ such that $(\mathrm{id} \otimes f) U^{\varphi(x)}=Z_{12}^{\varphi(x)} X_{13}^{x}, x \in \operatorname{Irred}\left(\mathbb{G}_{1}\right)$ it is easy to see that

$$
\begin{aligned}
& \left(\operatorname{id}_{\mathcal{H}_{\varphi^{\prime}(x)}} \otimes f^{-1}\right)\left(\operatorname{id}_{\mathcal{H}_{\varphi^{\prime}(x)}} \otimes \operatorname{id}_{\tilde{\mathcal{B}}} \otimes \varepsilon \otimes \operatorname{id}_{\mathcal{B}}\right) V^{\varphi^{\prime}(x)} \\
& =\left(\operatorname{id}_{\mathcal{H}_{\varphi^{\prime}(x)}} \otimes f^{-1}\right)\left(\operatorname{id} \otimes \operatorname{id}_{\tilde{\mathcal{B}}} \otimes \varepsilon \otimes \operatorname{id}_{\mathcal{B}}\right)\left(Z_{12}^{\varphi\left(x_{\mathbb{G}_{1}}\right)} U_{13}^{x} X_{14}^{x_{\mathbb{G}_{1}}}\right) \\
& =\left(\operatorname{id}_{\mathcal{H}_{\varphi^{\prime}(x)}} \otimes f^{-1}\right)\left(Z_{12}^{\varphi\left(x_{\mathbb{G}_{1}}\right)} X_{13}^{x_{\mathbb{G}_{1}}}\right) \\
& =\oplus_{i}\left(\operatorname{id}_{\mathcal{H}_{\varphi^{\prime}(x)}} \otimes f^{-1}\right)\left(Z_{12}^{\varphi\left(y_{i}\right)} X_{13}^{y_{i}}\right) \\
& =\oplus_{i} U^{\varphi\left(y_{i}\right)}=U^{\varphi\left(x_{\mathbb{G}_{1}}\right)}=\left(\operatorname{id}_{\mathcal{H}_{\varphi^{\prime}(x)}} \otimes \pi^{\prime}\right) V^{\varphi^{\prime}(x)}
\end{aligned}
$$

if $U^{x_{\mathbb{G}_{1}}}=\oplus_{i} U^{y_{i}}$. Hence, $\left(\mathrm{id} \otimes f^{-1}\right)\left(\mathrm{id} \otimes \mathrm{id}_{\tilde{\mathcal{B}}} \otimes \varepsilon \otimes \mathrm{id}_{\mathcal{B}}\right)=\left(\mathrm{id} \otimes \pi^{\prime}\right)$, proving that $\pi$ is multiplicative as composition of algebra maps. This concludes the proof.

Finally, we prove that the two monoidal equivalences $\varphi$ and $\varphi^{\prime}$ make isomorphic deformed spectral triples.

Proposition 6.15 Let $\mathbb{G}_{1}, \mathbb{G}_{2}, \mathbb{H}_{1}$ be compact quantum groups such that $\mathbb{G}_{1}$ is a compact quantum subgroup of $\mathbb{H}_{1}$ with surjective morphism $\pi: C_{u}\left(\mathbb{H}_{1}\right) \rightarrow C_{u}\left(\mathbb{G}_{1}\right)$ and let $\varphi: \mathbb{G}_{1} \rightarrow \mathbb{G}_{2}$ be a monoidal equivalence as above. Let $\mathbb{H}_{2}$ and $\varphi^{\prime}$ be the 
compact quantum group and monoidal equivalence induced by $\varphi$ as in Proposition 6.12. Suppose $\mathbb{H}_{1}$ resp. $\mathbb{G}_{1}$ acts algebraically and by orientation-preserving isometries with a unitary representation $V$ resp. $U$ on a spectral triple $(\mathcal{A}, \mathcal{H}, D)$ such that $U=(\mathrm{id} \otimes \pi) V$. Denoting by $\mathcal{B}$ the $\left(\mathbb{G}_{1}-\mathbb{G}_{2}\right)$-bi-Galois object associated with $\varphi$, by $\tilde{\mathcal{B}}$ the $\left(\mathbb{G}_{2}-\mathbb{G}_{1}\right)$-bi-Galois object associated with $\varphi^{-1}$ and by $\mathcal{B}^{\prime}$ the $\left(\mathbb{H}_{1}-\mathbb{H}_{2}\right)$-bi-Galois object associated with $\varphi^{\prime}$, the deformed spectral triples

$$
\left(\mathcal{A} \underset{\mathcal{O}\left(\mathbb{G}_{1}\right)}{\dot{\square}} \mathcal{B}, \mathcal{H} \underset{C\left(\mathbb{G}_{1}\right)}{\otimes} L^{2}(\mathcal{B}), \tilde{D}\right)
$$

and

$$
\left(\mathcal{A} \underset{\mathcal{O}\left(\mathbb{H}_{1}\right)}{\square} \mathcal{B}^{\prime}, \mathcal{H} \underset{C\left(\mathbb{H}_{1}\right)}{\otimes} L^{2}\left(\mathcal{B}^{\prime}\right), \tilde{D}^{\prime}\right),
$$

(where $\tilde{D}^{\prime}$ is the deformation of $D$ along $\varphi^{\prime}$ ) are isomorphic.

Proof It is easy to see that the map

$$
\lambda: \mathcal{A} \underset{\mathcal{O}\left(\mathbb{G}_{1}\right)}{\square} \mathcal{B} \rightarrow \mathcal{A} \underset{\mathcal{O}\left(\mathbb{H}_{1}\right)}{\square} \mathcal{O}\left(\mathbb{H}_{1}\right) \underset{\mathcal{O}\left(\mathbb{G}_{1}\right)}{\square} \mathcal{B}: z \mapsto\left(\alpha_{V} \otimes \mathrm{id}_{\mathcal{B}}\right) z_{13}
$$

is an isomorphism of ${ }^{*}$-algebras with inverse $\left(\mathrm{id}_{\mathcal{A}} \otimes \varepsilon_{\mathbb{H}_{1}} \otimes \mathrm{id}_{\mathcal{B}}\right)$. Moreover, let $\phi: \mathcal{H} \underset{C\left(\mathbb{G}_{1}\right)}{\otimes} L^{2}(\mathcal{B}) \rightarrow \mathcal{H} \underset{C\left(\mathbb{H}_{1}\right)}{\otimes} L^{2}\left(\mathcal{O}\left(\mathbb{H}_{1}\right) \underset{\mathcal{O}\left(\mathbb{G}_{1}\right)}{\square} \mathcal{B}\right): \eta \rightarrow V_{12} \eta_{13}$. Then defining $\phi^{\prime}: \mathcal{H} \underset{C\left(\mathbb{H}_{1}\right)}{\otimes} L^{2}\left(\mathcal{O}\left(\mathbb{H}_{1}\right) \underset{\mathcal{O}\left(\mathbb{G}_{1}\right)}{\square_{1}} \mathcal{B}\right) \rightarrow \mathcal{H} \underset{C\left(\mathbb{G}_{1}\right)}{\otimes} L^{2}(\mathcal{B}): \xi \mapsto\left(\mathrm{id} \otimes h_{\mathbb{H}_{1}} \otimes \mathrm{id}\right) V_{12}^{*} \xi$,

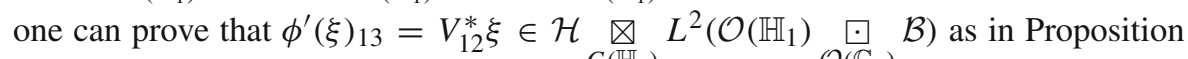
3.7. Hence, it follows that $\phi^{\prime}=\phi^{-1}$. Moreover, $\phi \tilde{D}=\stackrel{\mathcal{O}\left(\mathbb{G}_{1}\right)}{\tilde{D}^{\prime} \phi}$. Finally, we have for $z \in \mathcal{A} \underset{\mathcal{O}\left(\mathbb{G}_{1}\right)}{\square} \mathcal{B}$ and $\eta \in \mathcal{H} \underset{C\left(\mathbb{G}_{1}\right)}{\otimes} L^{2}(\mathcal{B})$

$$
\begin{aligned}
\lambda(z) \phi(\eta) & =V_{12} z_{13} V_{12}^{*} V_{12} \eta_{13} \\
& =V_{12} z_{13} \eta_{13} \\
& =\phi(z \eta)
\end{aligned}
$$

completing the proof.

\subsection{Deformation of the quantum isometry group}

\subsubsection{Deformation of the universal object in $\mathcal{Q}_{R}(\mathcal{A}, \mathcal{H}, D)$}

In this paragrah, we will investigate how the universal objects in the category $\mathcal{Q}_{R}(\mathcal{A}, \mathcal{H}, D)$ behave with respect to our deformation procedure.

Proposition 6.16 Let $R$ be a positive invertible operator such that $(\mathcal{A}, \mathcal{H}, D)$ is a $R$-twisted spectral triple. Let $\mathbb{G}_{1}$ be a compact quantum group acting algebraically 
and by orientation-preserving isometries on $(\mathcal{A}, \mathcal{H}, D)$ with a representation $U$ and suppose $\varphi: \mathbb{G}_{1} \rightarrow \mathbb{G}_{2}$ is a monoidal equivalence. Denote by $(\tilde{\mathcal{A}}, \tilde{\mathcal{H}}, \tilde{D})$ the deformed spectral triple (Theorem 3.12). Then there exists a positive invertible operator $\tilde{R}$ such that $(\tilde{\mathcal{A}}, \tilde{\mathcal{H}}, \tilde{D})$ is an $\tilde{R}$-twisted spectral triple on which $\mathbb{G}_{2}$ acts by $\tilde{R}$-twisted volumeand orientation-preserving isometries. Moreover, applying the same construction to $\varphi^{-1}$, we obtain $R$ again.

Proof We can decompose $\mathcal{H}$ as

$$
\mathcal{H}=\oplus_{x \in \operatorname{Irred}\left(\mathbb{G}_{1}\right)} \mathcal{H}_{x} \otimes W_{x}
$$

for some Hilbert spaces $W_{x}$, where the direct sum is taken over all $x \in \operatorname{Irred}\left(\mathbb{G}_{1}\right)$, all with multiplicity one. As $D$ commutes with the representation $U, D$ is of the form $D=\oplus_{x \in \operatorname{Irred}\left(\mathbb{G}_{1}\right)} \mathrm{id}_{\mathcal{H}_{x}} \otimes D_{x}$ where the $D_{x}$ are operators $W_{x} \rightarrow W_{x}$. As $\mathbb{G}_{1}$ acts by $R$-twisted volume-preserving isometries,

$$
\left(\tau_{R} \otimes \mathrm{id}\right)\left(\alpha_{U}(x)\right)=\tau_{R}(x) 1_{C(\mathbb{G})}
$$

for all $x \in \mathcal{E}_{D}$, where $\tau_{R}(x)=\operatorname{Tr}(R x)$ and $\mathcal{E}_{D}$ is the ${ }^{*}$-subalgebra of $B(\mathcal{H})$ generated by the rank-one operators of the form $\eta \xi^{*}, \eta, \xi$ eigenvectors of $D$. Therefore, also $\left(\tau_{R} \otimes h_{\mathbb{G}_{1}}\right)\left(\alpha_{U}(x)\right)=\tau_{R}(x)$ from which it follows (as in the proof of theorem 3.8 of [14]) that $R$ must be of the form $R=\oplus_{x \in \operatorname{Irred}\left(\mathbb{G}_{1}\right)} F_{x}^{\mathrm{T}} \otimes R_{x}$, where $F_{x}$ is the matrix such that $h_{\mathbb{G}_{1}}\left(u_{i j}^{x}\left(u_{s t}^{y}\right)^{*}\right)=\frac{\delta_{x, y} \delta_{i, s}\left(F_{x}\right)_{j t}}{\operatorname{Tr}\left(F_{x}\right)}$ (described by Woronowicz [26]) and $R_{x}: W_{x} \rightarrow$ $W_{x}$ positive operators. As $(\mathcal{A}, \mathcal{H}, D)$ is an $R$-twisted spectral triple, $R$ and $D$ commute and hence each $D_{x}$ commutes with $R_{x}$ for all $x \in \operatorname{Irred}\left(\mathbb{G}_{1}\right)$. Now, in this presentation, $\tilde{\mathcal{H}}=\oplus_{x \in \operatorname{Irred}\left(\mathbb{G}_{1}\right)} \mathcal{H}_{\varphi(x)} \otimes W_{x}$ and $\tilde{D}=\oplus_{x \in \operatorname{Irred}\left(\mathbb{G}_{1}\right)} \operatorname{id}_{\mathcal{H}_{\varphi(x)}} \otimes D_{x}$. Therefore, define $\tilde{R}=\oplus_{x \in \operatorname{Irred}\left(\mathbb{G}_{1}\right)} F_{\varphi(x)}^{\mathrm{T}} \otimes R_{x}$. Then, $\tilde{R}$ is again positive and invertible and it commutes with $\tilde{D}$. Moreover, $\mathbb{G}_{2}$ acts by $\tilde{R}$-twisted volume-preserving isometries by the defining property of $F_{\varphi(x)}$. It is clear that the inverse construction gives $R$ again.

Theorem 6.17 Let $R$ be a positive invertible operator on a Hilbert space $\mathcal{H}$ and let $(\mathcal{A}, \mathcal{H}, D)$ be an $R$-twisted compact spectral triple on which $\operatorname{QISO}_{R}^{0}(\mathcal{A}, \mathcal{H}, D)$ acts algebraically. Suppose $\varphi: \mathrm{QISO}_{R}^{0}(\mathcal{A}, \mathcal{H}, D) \rightarrow \mathbb{G}_{2}$ is a monoidal equivalence with bi-Galois object $\mathcal{B}$. Then, $\mathbb{G}_{2} \cong \operatorname{QISO}_{\tilde{R}}^{0}(\tilde{\mathcal{A}}, \tilde{\mathcal{H}}, \tilde{D})$ for $\tilde{R}$ as in Proposition 6.16 .

Remark 6.18 Note that the condition that $\operatorname{QISO}_{R}^{0}(\mathcal{A}, \mathcal{H}, D)$ acts algebraically on $(\mathcal{A}, \mathcal{H}, D)$ is not essential. If $\operatorname{QISO}_{R}^{0}(\mathcal{A}, \mathcal{H}, D)$ does not act algebraically on $(\mathcal{A}, \mathcal{H}, D)$, we know from Proposition 3.4 that there exists a ${ }^{*}$-algebra $\mathcal{A}_{1}$ which is SOT-dense in $\mathcal{A}^{\prime \prime}$ such that $\left(\mathcal{A}_{1}, \mathcal{H}, D\right)$ is a compact spectral triple on which $\operatorname{QISO}_{R}^{0}(\mathcal{A}, \mathcal{H}, D)$ acts algebraically. Moreover, $\operatorname{QISO}_{R}^{0}(\mathcal{A}, \mathcal{H}, D) \cong$ $\mathrm{QISO}_{R}^{0}\left(\mathcal{A}_{1}, \mathcal{H}, D\right)$ by proposition 3.9 of [14].

Proof of theorem 6.17 By Proposition 6.4, there exists a universal object $\mathrm{QISO}_{R}^{0}(\mathcal{A}, \mathcal{H}, D)$ in the category $\mathcal{Q}_{R}$ of compact quantum groups acting by $R$-twisted volume- and orientation-preserving isometries on $(\mathcal{A}, \mathcal{H}, D)$. For notational convenience, we will denote this quantum group by $\mathrm{QISO}_{\mathrm{R}}^{0}$. Now, as $\varphi: \mathrm{QISO}_{\mathrm{R}}^{0} \rightarrow \mathbb{G}_{2}$ is 
a monoidal equivalence, $\mathbb{G}_{2}$ acts algebraically and by orientation-preserving isometries on $(\tilde{\mathcal{A}}, \tilde{\mathcal{H}}, \tilde{D})=\left(\mathcal{\mathcal { A }} \underset{\mathcal{O}\left(\mathrm{QISO}_{\mathrm{R}}^{0}\right)}{\square} \mathcal{B}, \mathcal{H} \underset{C\left(\mathrm{QISO}_{\mathrm{R}}^{0}\right)}{\otimes} L^{2}(\mathcal{B}), \tilde{D}\right)$. Denote by $\tilde{R}$ the operator constructed in Proposition 6.16. Then $\mathbb{G}_{2}$ is a quantum subgroup of $\operatorname{QISO}_{\tilde{R}}^{0}(\tilde{\mathcal{A}}, \tilde{\mathcal{H}}, \tilde{D})$. Moreover, the monoidal equivalence $\varphi^{-1}: \mathbb{G}_{2} \rightarrow \operatorname{QISO}_{R}^{0}(\mathcal{A}, \mathcal{H}, D)$ induces a unitary fiber functor $\psi^{\prime}$ on $\operatorname{QISO}_{\tilde{R}}^{0}(\tilde{\mathcal{A}}, \tilde{\mathcal{H}}, \tilde{D})$ by Proposition 6.12; we will denote the deformed quantum group by $\mathbb{H}_{1}$ and the monoidal equivalence associated with $\psi^{\prime}$ (for notational convenience) by $\varphi^{\prime-1}: \operatorname{QISO}_{\tilde{R}}^{0}(\mathcal{A}, \mathcal{H}, D) \rightarrow \mathbb{H}_{1}$ and the associated bi-Galois object by $\tilde{\mathcal{B}}^{\prime}$. As $\mathbb{G}_{2}$ is a quantum subgroup of $\operatorname{QISO}_{\tilde{R}}^{0}(\tilde{\mathcal{A}}, \tilde{\mathcal{H}}, \tilde{D}), \operatorname{QISO}_{R}^{0}(\mathcal{A}, \mathcal{H}, D)$ is a quantum subgroup of $\mathbb{H}_{1}$ by Proposition 6.14 and both act by $R$-twisted volumeand orientation-preserving isometries on $(\mathcal{A}, \mathcal{H}, D)$ by Proposition 6.15 . Hence by universality,

$$
\mathrm{QISO}_{R}^{0}\left(\mathcal{A}_{1}, \mathcal{H}, D\right) \cong \mathbb{H}_{1}
$$

and also

$$
\mathbb{G}_{2} \cong \operatorname{QISO}_{R}^{0}(\tilde{\mathcal{A}}, \tilde{\mathcal{H}}, \tilde{D})
$$

This completes the proof.

\subsubsection{Deformation of the quantum isometry group}

In this paragraph, we use Sect. 6.1 and paragraph 6.3.1 to strengthen the result of Theorem 6.17 to quantum isometry groups.

Theorem 6.19 Let $(\mathcal{A}, \mathcal{H}, D)$ be an $R$-twisted compact spectral triple such that $\mathrm{QISO}_{R}^{0}(\mathcal{A}, \mathcal{H}, D)$ acts algebraically on $(\mathcal{A}, \mathcal{H}, D)$. Suppose moreover that we have a monoidal equivalence

$$
\varphi: \operatorname{QISO}_{R}^{0}(\mathcal{A}, \mathcal{H}, D) \rightarrow \mathbb{G}_{2}
$$

Then there exists a monoidal equivalence

$$
\varphi^{\prime}: \operatorname{QISO}_{R}(\mathcal{A}, \mathcal{H}, D) \rightarrow \operatorname{QISO}_{\tilde{R}}(\tilde{\mathcal{A}}, \tilde{\mathcal{H}}, \tilde{D}),
$$

where $(\tilde{\mathcal{A}}, \tilde{\mathcal{H}}, \tilde{D})$ is the spectral triple obtained by deformation with $\varphi$ by Theorem 3.12 and $\tilde{R}$ the operator obtained from Proposition 6.16 .

Remark 6.20 One can make again Remark 6.18 here.

Proof of theorem 6.19 Denote the universal object of $\mathcal{Q}_{R}$ for notational convenience by $\left.\mathrm{QISO}_{\mathrm{R}}^{0}=\left(C(\mathrm{QISO})_{\mathrm{R}}^{0}\right), U_{0}\right)$. Analogously, $\mathrm{QISO}_{\tilde{R}}^{0}=\mathrm{QISO}_{\tilde{R}}^{0}(\tilde{\mathcal{A}}, \tilde{\mathcal{H}}, \tilde{D})$. As $C\left(\mathrm{QISO}_{R}\right)=C^{*}\left(\left\{(f \otimes \mathrm{id}) \alpha_{U}(a) \mid a \in \mathcal{A}, f \in \mathcal{A}^{*}\right\}\right)$, it is a Woronowicz $C^{*}$ subalgebra of $\mathrm{QISO}_{\mathrm{R}}^{0}$ and hence we can apply the theory of section 6.1. We obtain a compact quantum group $\mathbb{H}_{2}$ and a monoidal equivalence $\varphi^{\prime}: \mathrm{QISO}_{R} \rightarrow \mathbb{H}_{2}$ 
and it suffices to prove $\mathbb{H}_{2}=\operatorname{QISO}_{\tilde{R}}(\tilde{\mathcal{A}}, \tilde{\mathcal{H}}, \tilde{D})$. Note now that as $\operatorname{QISO}_{\mathrm{R}}^{0}$ acts algebraically on $(\mathcal{A}, \mathcal{H}, D)$, we can decompose $\mathcal{A}$ into spectral subspaces $\mathcal{A}_{x}$ and define the subset $I$ of $\operatorname{Irred}\left(\mathrm{QISO}_{\mathrm{R}}^{0}\right)$ by $I=\left\{x \in \operatorname{Irred}\left(\mathrm{QISO}_{\mathrm{R}}^{0}\right) \mid \mathcal{A}_{x} \neq 0\right\}$. Then we have $C\left(\mathrm{QISO}_{R}\right)=C^{*}\left(\left\{u_{i j}^{x} \mid x \in I\right\}\right)$ and $I=\operatorname{Irred}\left(\mathrm{QISO}_{R}\right)$. Moreover, $C\left(\mathbb{H}_{2}\right)=C^{*}\left(\left\{u_{i j}^{\varphi(x)} \mid x \in I\right\}\right)$ and by theorem 7.3 of [11], we know that also $I=\left\{x \in \operatorname{Irred}\left(\mathrm{QISO}_{\mathrm{R}}^{0}\right) \mid \tilde{\mathcal{A}}_{\varphi(x)} \neq 0\right\}$. Hence, we can conclude that $\mathbb{H}_{2}=\operatorname{QISO}_{\tilde{R}}(\tilde{\mathcal{A}}, \tilde{\mathcal{H}}, \tilde{D})$.

This concludes the proof.

\subsection{Deformation of the quantum isometry group of the Podleś sphere}

In this last subsection, we use Sect. 6.3 to find the quantum isometry group of the newly constructed spectral triple in Theorem 5.10. Therefore, we investigate first the quantum isometry group of the Podleś sphere.

Definition 6.21 ([17]) Define $B$ to be the unital *-subalgebra of $C\left(S U_{q}(2)\right)$ generated (as *-algebra) by the elements $\alpha^{2}, \gamma^{*} \gamma, \gamma^{2}, \alpha \gamma$ and $\gamma^{*} \alpha$. The closure of $B$ is a Woronowicz $C^{*}$-algebra of $S U_{q}(2)$ and the associated compact quantum group is called $\mathrm{SO}_{q}(3)$.

In the classical situation, we know that $S O(3)$ is a quotient group of $S U(2)$; indeed, $S O(3)=S U(2) /\{-1,1\}$. In the quantum versions this is also true: we can prove that $\mathbb{Z}_{2}$ is a normal quantum subgroup of $S U_{q}(2)$ and $S U_{q}(2) / \mathbb{Z}_{2}$ equals $S O_{q}(3)$.

Theorem 6.22 ([5]) Let $S_{q, c}^{2}$ be the Podleś sphere as defined in Sect. 5.2. Then,

$$
\mathrm{QISO}_{R}\left[\mathcal{O}\left(S_{q, c}^{2}\right), \mathcal{H}, D\right] \cong S O_{q}(3) \text {. }
$$

Now, we will investigate how the monoidal equivalences of $S O_{q}(3)$ are induced by those of $S O_{q}(2)$ to apply Theorem 6.19 to find the quantum isometry group of the spectral triples constructed in Theorem 5.10.

We defined $S O_{q}(3)$ as coming from a Woronowicz- $C^{*}$-subalgebra of $S U_{q}(2)$. Using the theorems of Sect. 6.1, we will use the induction method to construct monoidal equivalences on $S O_{q}(3)$. Therefore, fix a monoidal equivalence between $S U_{q}(2)$ and a suitable $A_{o}\left(F^{\prime}\right)$ with $\operatorname{dim}\left(F^{\prime}\right) \geq 3$. As $S O_{q}(3)=S U_{q}(2) / \mathbb{Z}_{2}$, we find a Woronowicz subalgebra $I\left(F^{\prime}\right)$ of $A_{o}\left(F^{\prime}\right)$ such that $S O_{q}(3)$ is monoidally equivalent to $I\left(F^{\prime}\right)$. Now Theorem 4.1 in [23] gives us a concrete description of $I\left(F^{\prime}\right)$.

Theorem 6.23 (Theorem 4.1 in [23]) Let $F \in \mathrm{GL}(n, \mathbb{C})$ be such that $F \bar{F}= \pm I_{n}$. Then every Woronowicz subalgebra of $A_{o}(F)$ is a quantum quotient group. Moreover, it has only one normal subgroup of order 2 with quantum quotient group $C^{*}\left(r_{2 m}\right)$ (where $r_{2 m}$ is as in the parametrization of Banica [2]).

Applying this theorem to $F=F_{q}$, it affirms that $S O_{q}(3)$ is the only compact quantum quotient group of $S U_{q}(2)$. Applying it to $F=F^{\prime}$, we get a concrete description of $I\left(F^{\prime}\right)$. By Remark 5.8, it can be seen that the induced monoidal equivalence is not dimension preserving and hence not a 2-cocycle deformation (by Proposition 4.2).

Combining all of this, we get: 
Theorem 6.24 Let $F \in \mathrm{GL}(n, \mathbb{C})$ be such that $F \bar{F}= \pm I_{n}$ and $\varphi: S U_{q}(2) \rightarrow A_{o}(F)$ a monoidal equivalence with bi-Galois object $\mathcal{B}=A_{o}\left(F_{q}, F\right)$. Define $I(F)$ to be the $C^{*}$-algebra generated by the $U_{i j} U_{k l}$ where $U$ is the unitary in $M_{n}\left(A_{o}(F)\right)$ satisfying the relation $U=F \bar{U} F^{-1}$ as in Definition 5.1. Define $P\left(F_{q}, F\right)$ to be the *algebra generated by the $Y_{i j} Y_{k l}$ where $Y$ is the unitary in $M_{n, 2}(\mathbb{C}) \otimes \mathcal{O}\left(A_{o}\left(F_{q}, F\right)\right)$ described in Theorem 5.7. Then there exists a monoidal equivalence $\varphi^{\prime}: S O_{q}(3) \rightarrow I(F)$ with bi-Galois object $\mathcal{B}^{\prime}=P\left(F_{q}, F\right)$ which is not dimension preserving (by Remark 5.8).

Now, we are ready to characterize the quantum isometry groups of the spectral triples constructed in Theorem 5.10.

Theorem 6.25 Let $q \in[-1,1] \backslash\{0\}$ and $n$ a natural number with $3 \leq n \leq|q+1 / q|$. If $q>0$, suppose $n$ is even. With the matrix $F$ defined as in Theorem 5.10, I $(F)$ as constructed in Theorem 6.24 is the quantum isometry group of the spectral triple

$$
\left(\mathcal{O}\left(S_{q, c}^{2}\right) \underset{\mathcal{O}\left(S U_{q}(2)\right)}{\ominus} \mathcal{O}\left(A_{o}\left(F_{q}, F\right)\right), \quad \mathcal{H} \underset{C\left(S U_{q}(2)\right)}{\otimes} L^{2}\left(\mathcal{O}\left(A_{o}\left(F_{q}, F\right)\right)\right), \quad \tilde{D}\right)
$$

from Theorem 5.10 .

Acknowledgements I thank Johan Quaegebeur and Pierre Bieliavsky for many fruitful discussions and most valuable and appreciated advice. Also, Kenny De Commer, Yuki Arano and Stefaan Vaes are warmly thanked for discussing about the subject. The valuable and detailed comments and suggestions of the referees are warmly appreciated. They made it possible to improve the paper substantially. Finally, I thank the IAP DYGEST for financial support.

Open Access This article is distributed under the terms of the Creative Commons Attribution 4.0 International License (http://creativecommons.org/licenses/by/4.0/), which permits unrestricted use, distribution, and reproduction in any medium, provided you give appropriate credit to the original author(s) and the source, provide a link to the Creative Commons license, and indicate if changes were made.

\section{References}

1. Baaj, S., Skandalis, G.: Unitaires multiplicatifs et dualité pour les produits croisés de C*-algèbres. Ann. Scient. Ec. Norm. Sup. 4, 425-488 (1993)

2. Banica, T.: Theorie des representations du groupe quantique compact libre O(n). C.R. Acad. Sci. Paris Sér. I Math. 322, 241-244 (1996)

3. Bédos, E., Murphy, G., Tuset, L.: Co-amenability of compact quantum groups. J. Geom. Phys. 40(2), 129-153 (2001)

4. Bhowmick, J., Goswami, D.: Quantum group of orientation-preserving Riemannian isometries. J. Funct. Anal. 257(8), 2530-2572 (2009)

5. Bhowmick, J., Goswami, D.: Quantum isometry groups of the Podles spheres. J. Funct. Anal. 258(9), 2937-2960 (2010)

6. Bichon, J., De Rijdt, A., Vaes, S.: Ergodic coactions with large multiplicity and monoidal equivalence of quantum groups. Commun. Math. Phys. 262(3), 703-728 (2005)

7. Boca, F.: Ergodic actions of compact matrix pseudogroups on $C^{*}$-algebras. Astérisque 232, 93-109 (1995)

8. Connes, A.: Noncommutative Geometry. Academic Press, San Diego (1994)

9. Connes, A., Landi, G.: Noncommutative manifolds the instanton algebra and isospectral deformations. Commun. Math. Phys. 159, 20 (2000)

10. Dabrowski, L., D’Andrea, F., Landi, G., Wagner, E.: Dirac operators on all Podles quantum spheres. J. Noncommut. Geometry 1(2), 213-239 (2007) 
11. De Rijdt, A., Vander Vennet, N.: Actions of monoidally equivalent compact quantum groups and applications to probabilistic boundaries. Annales de L'Institut Fourier 60(1), 169-216 (2010)

12. Goswami, D.: Twisted entire cyclic cohomology, J-L-O cocycles and equivariant spectral triples. Rev. Math. Phys. 16(5), 583-602 (2004). doi:10.1142/S0129055X04002114

13. Goswami, D.: Quantum group of isometries in classical and noncommutative geometry. Commun. Math. Phys. 285(1), 141-160 (2009)

14. Goswami, D., Joardar, S.: Quantum isometry groups of noncommutative manifolds obtained by deformation using dual unitary 2-cocycles. SIGMA Symmetry Integr. Geom. Methods Appl. 10, 076 (2014)

15. Maes, A., Van Daele, A.: Notes on compact quantum groups. Nieuw Arch. Wisk. IV. 16(1-2), 73-112 (1998)

16. Majid, S.: Foundations of Quantum Group Theory. Cambridge University Press, Cambridge (1995)

17. Podleś, P.: Quantum spheres. Lett. Math. Phys. 14(3), 193-202 (1987)

18. Podleś, P.: Symmetries of quantum spaces. Subgroups and quotient spaces of quantum SU(2) and SO(3) groups. Commun. Math. Phys. 170(1), 1-20 (1995)

19. Schauenburg, P.: Hopf-Galois and Bi-Galois extensions. Fields Inst. Commun. 34, 469-515 (2004)

20. Van Daele, A.: Discrete quantum groups. J. Algebra 180(2), 431-444 (1996)

21. Van Daele, A., Wang, S.: Universal quantum groups. Int. J. Math. 7(2), 255-263 (1996). doi:10.1142/ S0129167X96000153

22. Wang, S.: Free products of compact quantum groups. Commun. Math. Phys. 692, 671-692 (1995)

23. Wang, S.: Simple compact quantum groups I. J. Funct. Anal. 256(10), 3313-3341 (2009)

24. Woronowicz, S.L.: Compact matrix pseudogroups. Commun. Math. Phys. 111(4), 613-665 (1987)

25. Woronowicz, S.L.: Twisted SU(2) group. An example of a non-commutative differential calculus. Publ. RIMS Kyoto Univ. 23, 117-181 (1987)

26. Woronowicz, S.L.: Compact quantum groups. In: Symétries quantiques (Les Houches, 1995), vol. 64, pp. $845-884$ 